Report of the First Confidence Building Exercise For Biomedical Sample Analysis

Armando alcaraz, Hugh Gregg

February 26, 2010 
This document was prepared as an account of work sponsored by an agency of the United States government. Neither the United States government nor Lawrence Livermore National Security, LLC, nor any of their employees makes any warranty, expressed or implied, or assumes any legal liability or responsibility for the accuracy, completeness, or usefulness of any information, apparatus, product, or process disclosed, or represents that its use would not infringe privately owned rights. Reference herein to any specific commercial product, process, or service by trade name, trademark, manufacturer, or otherwise does not necessarily constitute or imply its endorsement, recommendation, or favoring by the United States government or Lawrence Livermore National Security, LLC. The views and opinions of authors expressed herein do not necessarily state or reflect those of the United States government or Lawrence Livermore National Security, LLC, and shall not be used for advertising or product endorsement purposes.

This work performed under the auspices of the U.S. Department of Energy by Lawrence Livermore National Laboratory under Contract DE-AC52-07NA27344. 


\title{
Report of the First Confidence Building Exercise for Biomedical Sample Analysis
}

\author{
Hugh R. Gregg, Armando Alcaraz \\ Physical and Life Sciences, LLNL
}

\section{Summary}

We participated in the first exercise to build confidence in the analysis of biomedical samples for trace levels of $\mathrm{CW}$ agents and/or their degradation, reaction or metabolites. The report is attached.

\section{DISCLAIMER}

This document was prepared as an account of work sponsored by an agency of the United States government. Neither the United States government nor Lawrence Livermore National Security, LLC, nor any of their employees makes any warranty, expressed or implied, or assumes any legal liability or responsibility for the accuracy, completeness, or usefulness of any information, apparatus, product, or process disclosed, or represents that its use would not infringe privately owned rights. Reference herein to any specific commercial product, process, or service by trade name, trademark, manufacturer, or otherwise does not necessarily constitute or imply its endorsement, recommendation, or favoring by the United States government or Lawrence Livermore National Security, LLC. The views and opinions of authors expressed herein do not necessarily state or reflect those of the United States government or Lawrence Livermore National Security, LLC, and shall not be used for advertising or product endorsement purposes.

This work performed under the auspices of the U.S. Department of Energy by Lawrence Livermore National Laboratory under Contract DE-AC52-07NA27344. 


\title{
ORGANISATION FOR THE PROHIBITION OF CHEMICAL WEAPONS
}

\section{Report of the \\ First Confidence Building Exercise For Biomedical Sample Analysis}

\author{
Laboratory code: $\quad 22$ \\ Total number of pages:
}

1 Total number of pages including cover page and all attachments 
Cover page

Page no.

Table of contents

Summary

Participating Laboratory .............................................. 3

Summary: Chemicals Identified \& \& Methods............................. 4

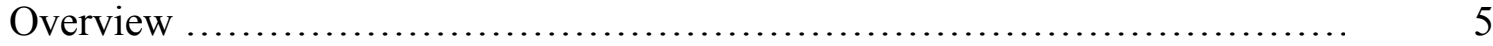

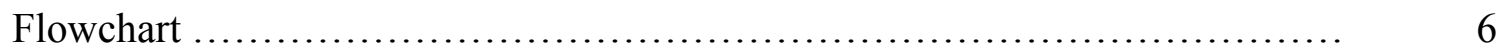

Detailed Sample Preperation............................................ 7

Detailed Analytical Methods ............................................ 9

\section{Results}

Urine Sample S2 ................................................. 12

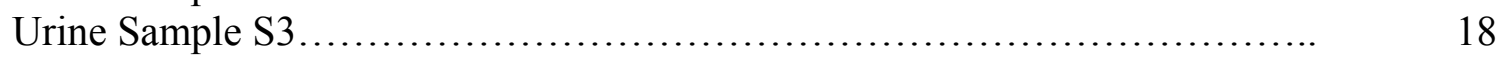

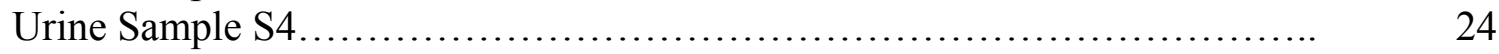

Urine Sample S5................................................... 25

Urine Sample S6.................................................. 34

Urine Sample S7 ............................................ 40

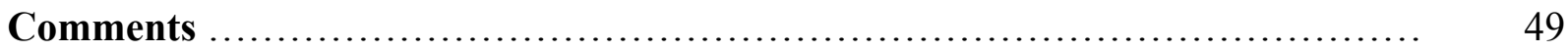




\section{SUMMARY: PARTICIPATING LABORATORY}

1. Participating laboratory

\begin{tabular}{|l|l|}
\hline Laboratory code: & 22 \\
\hline $\begin{array}{l}\text { Name of the } \\
\text { laboratory/institute } \\
\text { participating in the test: }\end{array}$ & Lawrence Livermore National Laboratory \\
\hline Contact person: & Mr. Armando Alcaraz \\
\hline Address: & $\begin{array}{l}\text { PO Box } 808, \mathrm{M} / \mathrm{S} \mathrm{L-091} \\
7000 \text { East Avenue } \\
\text { Livermore, CA 94551 }\end{array}$ \\
\hline Telephone number: & $925-423-6889$ \\
\hline Fax number: & $925-423-9014$ \\
\hline Email address: & Alcaraz1@1lnl.gov \\
\hline
\end{tabular}

\section{Analysts and authentication}

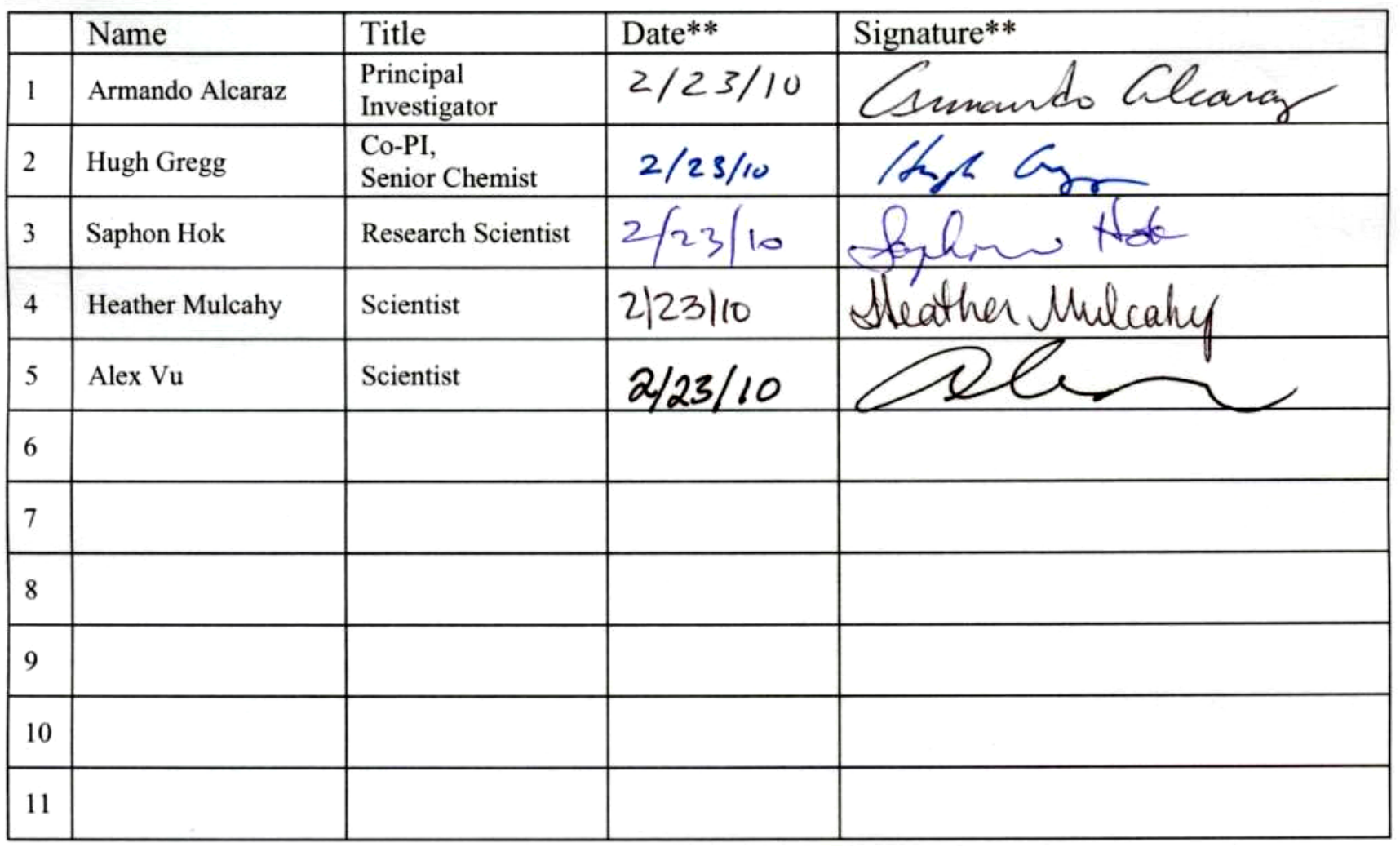

** Date and signature of the responsible analyst; 


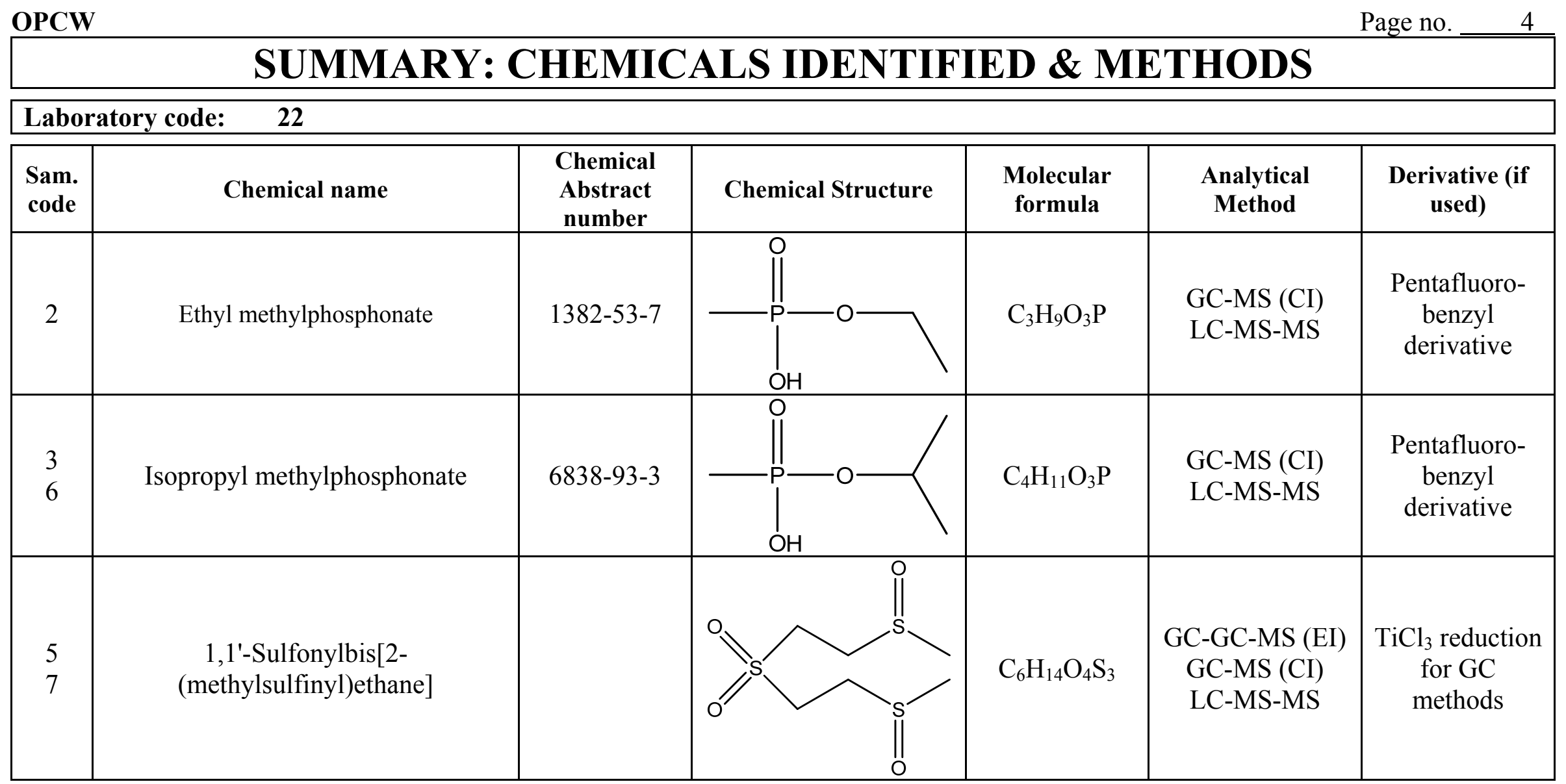

Chemical number defined by the participating laboratory and used throughout the report for the reported chemical.

Note: There must be an unbroken chain of evidence linking each reported chemical to the original sample from which an aliquot was prepared and analyzed for the identification of this chemical. 


\section{OVERVIEW}

LLNL received the following samples/reference standards for this exercise:

Tray/Box 1 contains: 7 vials with $\sim 3.3 \mathrm{~mL}$ each

Reference standard solutions provided to LLNL

\begin{tabular}{|l|l|l|l|}
\hline Sample & Matrix & Spiked agent & $\begin{array}{l}\text { Approximate } \\
\text { concentration } \\
(\mu \mathrm{g} / \mathrm{ml})\end{array}$ \\
\hline r1/22 & Water & EMPA & 10 \\
\hline r2/22 & Water & IMPA & 10 \\
\hline r3/22 & Water & PMPA & 10 \\
\hline r4/22 & Water & MPA & 10 \\
\hline r5/22 & Water & TDG & 10 \\
\hline r6/22 & Water & SBMSE & 10 \\
\hline r7/22 & Water & MSMTESE & 10 \\
\hline
\end{tabular}

EMPA $=$ O-ethyl methylphosphonic acid (Ethyl methylphosphonate)

IMPA = O-isopropyl methylphosphonic acid (Isopropyl methylphosphonate)

PMPA = O-pinacolyl methylphosphonic acid (Pinacolyl methylphosphonate)

MPA $=$ methylphosphonic acid

TDG $=$ thiodiglycol

SBMSE = 1,1'-Sulfonylbis[2-(methylsulfinyl)ethane]

MSMTESE = 1-Methylsulfinyl-2-[2-(methylthio)ethylsulfonyl]ethane

Tray/Box 2 contains: 24 vials with $\sim 3.3 \mathrm{~mL}$ each

\begin{tabular}{|l|l|l|l|l|l|l|}
\hline Blanks & Sample \#2 & Sample \#3 & Sample \#4 & Sample \#5 & Sample \#6 & Sample \#7 \\
\hline $\mathrm{S} 1 \mathrm{~A} / 22$ & $\mathrm{~S} 2 \mathrm{~A} / 22$ & $\mathrm{~S} 3 \mathrm{~A} / 22$ & $\mathrm{~S} 4 \mathrm{~A} / 22$ & $\mathrm{~S} 5 \mathrm{~A} / 22$ & $\mathrm{~S} 6 \mathrm{~A} / 22$ & $\mathrm{~S} 7 \mathrm{~A} / 22$ \\
\hline $\mathrm{S} 1 \mathrm{~B} / 22$ & $\mathrm{~S} 2 \mathrm{~B} / 22$ & $\mathrm{~S} 3 \mathrm{~B} / 22$ & $\mathrm{~S} 4 \mathrm{~B} / 22$ & $\mathrm{~S} 5 \mathrm{~B} / 22$ & $\mathrm{~S} 6 \mathrm{~B} / 22$ & $\mathrm{~S} 7 \mathrm{~B} / 22$ \\
\hline $\mathrm{S} 1 \mathrm{C} / 22$ & $\mathrm{~S} 2 \mathrm{C} / 22$ & $\mathrm{~S} 3 \mathrm{C} / 22$ & $\mathrm{~S} 4 \mathrm{C} / 22$ & $\mathrm{~S} 5 \mathrm{C} / 22$ & $\mathrm{~S} 6 \mathrm{C} / 22$ & $\mathrm{~S} 7 \mathrm{C} / 22$ \\
\hline $\mathrm{S} 1 \mathrm{D} / 22$ & & & & & & \\
\hline $\mathrm{S} 1 \mathrm{E} / 22$ & & & & & & \\
\hline $\mathrm{S} 1 \mathrm{~F} / 22$ & & & & & & \\
\hline
\end{tabular}

The task is to identify the acids and urinary metabolites of mustard which were spiked into the samples in the at the 0,10 or 100 ppb level.

Sample workup for all samples is the same, and follows the flowchart presented below. LC/MS and GC/MS were the analytical techniques employed.

Lab 22 currently does not have a method for the determination of low levels of thiodiglycol in urine. 


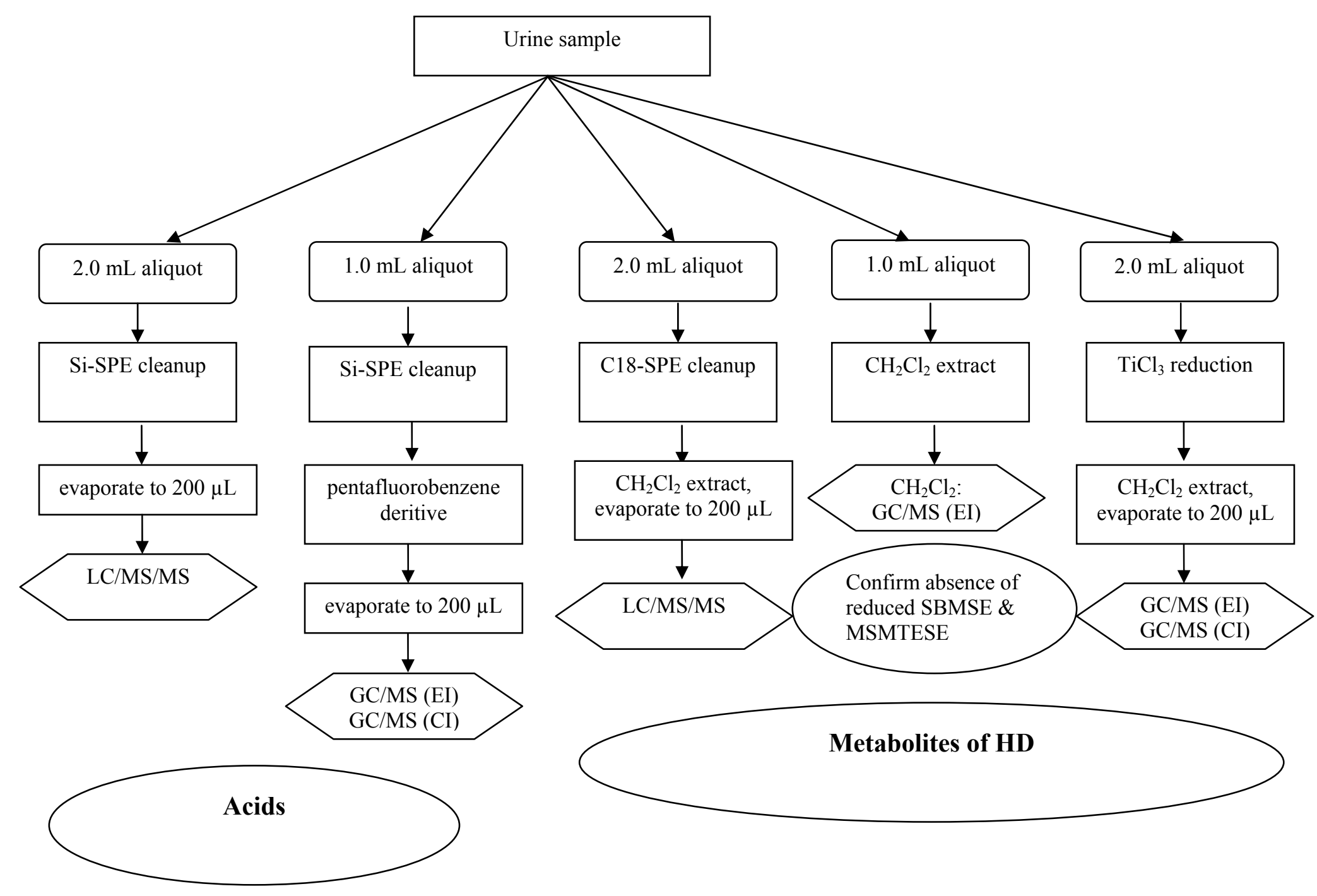




\section{Acids:}

\section{Prep for GC/MS:}

Silica SPE clean-up: This method similar to procedure used in Mawhinney(2007) and UK lab section 9.1. $1 \mathrm{~mL}$ of sample was aliquoted for work-up in a clean $4 \mathrm{~mL}$ sample vial. Sample was taken to dryness and the residue was extracted twice with $1.5 \mathrm{~mL}$ of $5 \% \mathrm{H}_{2} \mathrm{O}$ in Acetonitrile. A Supelco Discovery-Silica column $(500 \mathrm{mg} / 3 \mathrm{cc})$ was conditioned with $4.8 \mathrm{~mL}$ of $25 \% \mathrm{H}_{2} \mathrm{O}$ in acetonitrile followed by $3 \mathrm{~mL}$ of acetonitrile. Sample was taken through column and washed first with $2 \mathrm{~mL}$ of acetonitrile and followed with $4 \mathrm{~mL}$ of $10 \% \mathrm{H}_{2} \mathrm{O}$ in acetonitrile. Sample was eluted with $2.5 \mathrm{~mL}$ of $25 \% \mathrm{H}_{2} \mathrm{O}$ in acetonitrile into clean $4 \mathrm{~mL}$ vial.

Pentafluorobenzylation: Sample taken to dryness and $480 \mu \mathrm{L}$ of acetone was added to vial. Added to solution was $10 \mu \mathrm{L}$ of pentafluorobenzyl bromide and $10 \mu \mathrm{L}$ of diisopropylamine. Sample was placed on heat block and heated $75^{\circ} \mathrm{C}$ for 1 hour. Sample allowed to cool and volume reduced to approximately $200 \mu \mathrm{L}$ and transferred to vial insert.

Samples: S1, S2, S3, S4, S5, S6, S7

\section{Prep for LC/MS (for positive GC/MS samples only):}

Silica SPE clean-up: This method similar to procedure used in Mawhinney(2007) and UK lab section 9.1. $1 \mathrm{~mL}$ of sample was aliquoted for work-up in a clean $4 \mathrm{~mL}$ sample vial. Sample was taken to dryness and the residue was extracted twice with $1.5 \mathrm{~mL}$ of $5 \% \mathrm{H}_{2} \mathrm{O}$ in Acetonitrile. A Supelco Discovery-Silica column $(500 \mathrm{mg} / 3 \mathrm{cc})$ was conditioned with $4.8 \mathrm{~mL}$ of $25 \% \mathrm{H}_{2} \mathrm{O}$ in acetonitrile followed by $3 \mathrm{~mL}$ of acetonitrile. Sample was taken through column and washed first with $2 \mathrm{~mL}$ of acetonitrile and followed with $4 \mathrm{~mL}$ of $10 \% \mathrm{H}_{2} \mathrm{O}$ in acetonitrile. Sample was eluted with $2.5 \mathrm{~mL}$ of $25 \% \mathrm{H}_{2} \mathrm{O}$ in acetonitrile into clean $4 \mathrm{~mL}$ vial. Sample volume was reduced to approximately $200 \mu \mathrm{L}$.

Samples: S1, S2, S3, S6 


\section{HD metabolites:}

\section{Prep for GC/MS:}

Titanium Chloride reduction: $2 \mathrm{~mL}$ of sample was aliquoted for work-up in a clean $4 \mathrm{~mL}$ sample vial. To this vial, $2 \mathrm{~mL}$ of ca. $10 \%$ Titanium Chloride (in $\sim 20-30 \% \mathrm{HCl}$ ) was added and vortexed for 5 seconds. Sample vial was placed on heating block and sample heated to $70^{\circ} \mathrm{C}$ for 1 hour. Sample was then allowed to cool and liquid-liquid extraction performed using 3x $1 \mathrm{~mL}$ of DCM solvent. DCM extract was volume reduced using gentle stream of nitrogen to approximately $200 \mu \mathrm{L}$. Sample was transferred to vial insert.

Samples: S1, S2, S3, S4, S5, S6, S7

\section{Prep for LC/MS (for positive GC/MS samples only):}

C18 SPE clean-up: $2 \mathrm{~mL}$ of sample was aliquoted for work-up. An Alltech C18 column $(500 \mathrm{mg} / 3 \mathrm{cc})$ was conditioned with $3 \mathrm{~mL}$ of methanol, followed by $3 \mathrm{~mL}$ of HPLC water. $2 \mathrm{~mL}$ sample was taken through column and washed with $3 \mathrm{~mL}$ of water. Column was allowed to go to dryness and then proceeded to elute with $4 \mathrm{~mL}$ of DCM into clean sample vial. Elutant was taken to dryness and taken back up in 200uL of HPLC $\mathrm{H}_{2} \mathrm{O}$. Sample was transferred to vial insert. 


\section{DETAILED ANALYTICAL METHODS}

\section{GC-GC-MS (EI) parameters}

TOF-GC/MS Method Name: OPCW 2D CW122809

Instrument name: LECO Pegasus IV GCxGC

Carrier gas type: Helium

Flow rate: $1.20 \mathrm{~mL} / \mathrm{min}$

$\mathrm{X}$ Corrected constant flow via pressure ramps

Inlet type: Split/Splitless

Injection mode: Pulsed-splitless

Inlet purge time: $35 \mathrm{sec}$

Inlet purge flow: $30 \mathrm{~mL} / \mathrm{min}$

Inlet pulse pressure: $40 \mathrm{psi}$

Inlet pulse time: $0.5 \mathrm{~min}$

Inlet temperature: $250{ }^{\circ} \mathrm{C}$

Primary Column type/phase: Agilent HP-5MS (5\% diphenyl 95\% dimethyl polysiloxane)

Primary Column Length x ID x Film thickness: $15 \mathrm{~m}$ x $0.25 \mathrm{~mm}$ x $0.25 \mu \mathrm{m}$

Secondary Column type/phase: Restek Rxi-17 (50\% diphenyl 50\% dimethyl polysiloxane)

Secondary Column Length x ID x Film thickness: $1 \mathrm{~m}$ x $0.1 \mathrm{~mm}$ x $0.1 \mu \mathrm{m}$

Oven equilibration time: $0.5 \mathrm{~min}$

Primary Oven temperature program:

$70{ }^{\circ} \mathrm{C}(0.5 \mathrm{~min})$

$10{ }^{\circ} \mathrm{C} / \mathrm{min}$

$280{ }^{\circ} \mathrm{C}(3 \mathrm{~min})$

Secondary Oven temperature program:

$85^{\circ} \mathrm{C}(0.5 \mathrm{~min})$

$10{ }^{\circ} \mathrm{C} / \mathrm{min}$

$295^{\circ} \mathrm{C}(3 \mathrm{~min})$

Transfer line temperature: $295^{\circ} \mathrm{C}$

GCxGC parameters:

$\mathrm{X}$ Modulator enabled

Modulator temperature offset: $30^{\circ} \mathrm{C}$, relative to the $\mathrm{GC}$ oven temperature

Modulation period: $3 \mathrm{sec}$

Hot pulse time: $0.6 \mathrm{sec}$

Cool time between stages: $0.9 \mathrm{sec}$

MS parameters:

Acquisition delay: $90 \mathrm{sec}$

Scan range: $30-600 \mathrm{~m} / \mathrm{z}$

Acquisition rate: 200 spectra/second

Detector voltage: $1650 \mathrm{~V}$

Electron energy: $70 \mathrm{eV}$

Source temperature: $250{ }^{\circ} \mathrm{C}$ 


\section{GC-MS (CI) parameters}

\begin{tabular}{|c|c|}
\hline Parameter & Details \\
\hline GC instrument & Agilent 6890 \\
\hline $\begin{array}{l}\text { Column: } \\
\text { type: } \\
\text { dimensions }(\mathrm{m} \times \mathrm{mm}) \\
\text { film thickness }(\mu \mathrm{m})\end{array}$ & $\begin{array}{l}\text { Agilent HP5-MS } \\
30 \mathrm{~m} \times 0.25 \mathrm{~mm} \\
0.25 \mu \mathrm{m}\end{array}$ \\
\hline Guard column/retention gap & -- \\
\hline Carrier gas, flow rate $(\mathrm{cm} / \mathrm{s}$ or $\mathrm{psi})$ & $\mathrm{He}, 0.8 \mathrm{~mL} / \mathrm{min}, 32 \mathrm{~cm} / \mathrm{sec}$ \\
\hline Temperature program & $40^{\circ} \mathrm{C} / 3 \mathrm{~min}, 8^{\circ} \mathrm{C} / \mathrm{min}, 300^{\circ} \mathrm{C} / 3 \mathrm{~min}$ \\
\hline Injection type & Splitless \\
\hline Injector temperature $\left({ }^{\circ} \mathrm{C}\right)$ & $250^{\circ} \mathrm{C}$ \\
\hline Injection volume $(\mu \mathrm{l})$ & $1 \mu \mathrm{L}$ \\
\hline MS instrument (type) & Agilent 5973 \\
\hline Ionization type & $\mathrm{CI}-\mathrm{NH}_{3}$ for positive; $\mathrm{CH}_{4}$ for negative \\
\hline Emission current $(\mu \mathrm{A})$ & $242 \mu \mathrm{A}$ \\
\hline Electron energy $(\mathrm{eV})$ & $114 \mathrm{eV}$ \\
\hline Source temperature $\left({ }^{\circ} \mathrm{C}\right)$ & $150^{\circ} \mathrm{C}$ \\
\hline $\begin{array}{l}\text { Scan mode (MS full scan, SIM, MS-MS } \\
\text { full scan, MRM) }\end{array}$ & * \\
\hline Mass range (full scan) $(\mathrm{m} / \mathrm{z})$ & * \\
\hline Extracted ions (full scan) $(\mathrm{m} / \mathrm{z})$ & $*$ \\
\hline Selected ions (SIM) $(\mathrm{m} / \mathrm{z})$, r.t. window ${ }^{1}$ & * \\
\hline $\begin{array}{l}\text { Selected reactions }(\mathrm{MRM})(\mathrm{m} / \mathrm{z}) \text {, r.t. } \\
\text { window }^{1}\end{array}$ & * \\
\hline Resolution & $0.7 \mathrm{u}$ \\
\hline Interface/transfer line temperature $\left({ }^{\circ} \mathrm{C}\right)$ & $280^{\circ} \mathrm{C}$ \\
\hline Any additional parameters & \\
\hline
\end{tabular}

1. if appropriate

* Scan parameters are different for each analyte:

\begin{tabular}{|l|l|}
\hline Analyate & Scan parameters \\
\hline 1,1 '-Sulfonylbis[2-(methylthio)ethane] & Positive ion SIM: 232, 233, 234 \\
\hline Ethyl methylphosphonate & Negative ion SIM: 123 \\
\hline Isopropyl methylphosphonate & Negative ion SIM: 137 \\
\hline
\end{tabular}




\section{LC-MS-MS parameters}

\begin{tabular}{|c|c|c|}
\hline \multirow[t]{2}{*}{ Parameter } & \multicolumn{2}{|l|}{ Details } \\
\hline & HD metabolites & Acids \\
\hline LC instrument & \multicolumn{2}{|c|}{ LTQ XL-Orbitrap } \\
\hline $\begin{array}{l}\text { Column } \\
\text { Type } \\
\text { Dimensions }(\mathrm{mm}) \\
\text { Particle size }(\mu \mathrm{m})\end{array}$ & $\begin{array}{l}\text { Waters Sunfire } \\
\mathrm{C} 8 \\
2.1 \times 150 \mathrm{~mm} \\
3.5 \mu \mathrm{m}\end{array}$ & $\begin{array}{l}\text { Waters T3 } \\
\text { Unbonded Silica } \\
2.1 \times 150 \mathrm{~mm} \\
3 \mu \mathrm{m}\end{array}$ \\
\hline Oven temperature $\left({ }^{\circ} \mathrm{C}\right)$ & \multicolumn{2}{|l|}{$30^{\circ} \mathrm{C}$} \\
\hline Mobile phase & \multicolumn{2}{|c|}{$\begin{array}{l}\text { Solvent A: Water } \mathrm{w} / 0.1 \% \text { formic acid } \\
\text { Solvent B: ACN } / 0.1 \% \text { formic acid }\end{array}$} \\
\hline Gradient (or isocratic) & \multicolumn{2}{|c|}{$\begin{array}{l}\text { Gradient }(\% \mathrm{~B} / \mathrm{min}: 5 / 0,5 / 5,80 / 15,80 / 20 \text {, } \\
5 / 23,5 / 25)\end{array}$} \\
\hline \multicolumn{3}{|l|}{ Injection type } \\
\hline Injection volume $(\mu \mathrm{l})$ & \multicolumn{2}{|l|}{$5 \mu \mathrm{L}$} \\
\hline \multicolumn{3}{|l|}{ Loop volume $(\mu \mathrm{l})$} \\
\hline MS instrument & \multicolumn{2}{|c|}{ Thermo LTQ Orbitrap } \\
\hline Ionization, +ve / -ve & pos & Neg \\
\hline Spray voltage $(\mathrm{kV})$ & \multicolumn{2}{|l|}{$4 \mathrm{kV}$} \\
\hline \multicolumn{3}{|l|}{ Source CID voltage (V) } \\
\hline Source temperature $\left({ }^{\circ} \mathrm{C}\right)$ & \multicolumn{2}{|l|}{ Capillary $250^{\circ} \mathrm{C}$} \\
\hline \multicolumn{3}{|l|}{ Desolvation temperature $\left({ }^{\circ} \mathrm{C}\right)^{1}$} \\
\hline Desolvation gas, flow rate $^{\mid}$ & \multicolumn{2}{|l|}{20 (sheath gas) } \\
\hline Nebulizer gas, flow rate $^{1}$ & \multicolumn{2}{|l|}{5 (sweep gas) } \\
\hline $\begin{array}{l}\text { Scan mode (MS full scan, SIM, MS-MS } \\
\text { full scan, MRM) }\end{array}$ & \multicolumn{2}{|l|}{ * } \\
\hline Scan range (full scan) & \multicolumn{2}{|l|}{ * } \\
\hline Ions monitored (SIM), r.t. window $^{2}$ & \multicolumn{2}{|l|}{ * } \\
\hline Reactions monitored (MRM), r.t. window ${ }^{2}$ & \multicolumn{2}{|l|}{ * } \\
\hline Collision energy & 17 & 30 \\
\hline
\end{tabular}

1. or other relevant source parameters

2. if appropriate

* Scan parameters are different for each analyte:

\begin{tabular}{|l|l|}
\hline Analyate & Scan parameters \\
\hline $\begin{array}{l}\text { 1-Methylsulfinyl-2-[2- } \\
\text { (methylthio)ethylsulfonyl]ethane }\end{array}$ & $\begin{array}{l}\text { MS/MS } 253 \rightarrow(70 \text { to } 280) \\
\text { Products of }[\mathrm{M}+\mathrm{Na}]^{+}\end{array}$ \\
\hline $1,1^{\prime}-$ Sulfonylbis[2-(methylsulfinyl)ethane] & $\begin{array}{l}\text { MS/MS } 269 \rightarrow(70 \text { to } 280) \\
\text { Products of }[\mathrm{M}+\mathrm{Na}]^{+}\end{array}$ \\
\hline Ethyl methylphosphonate & $\begin{array}{l}\text { MS/MS } 123 \rightarrow(50 \text { to } 210) \\
\text { Products of }[\mathrm{M}-\mathrm{H}]^{-}\end{array}$ \\
\hline Isopropyl methylphosphonate & $\begin{array}{l}\text { MS/MS } 137 \rightarrow(50 \text { to } 210) \\
\text { Products of }[\mathrm{M}-\mathrm{H}]^{-}\end{array}$ \\
\hline
\end{tabular}




\section{RESULTS: URINE SAMPLE S2}

$\beta$-Lyase metabolites of sulfur mustard were not found in this sample (after sample preparation that included $\mathrm{TiCl}_{3}$ reduction and analysis by GC-GC-MS (EI)).

Ethyl methyphosophonate was found. The following few pages present the GC-MS (CI) data for the derivative and the LC-MS-MS data for the un-derivitized acid. 


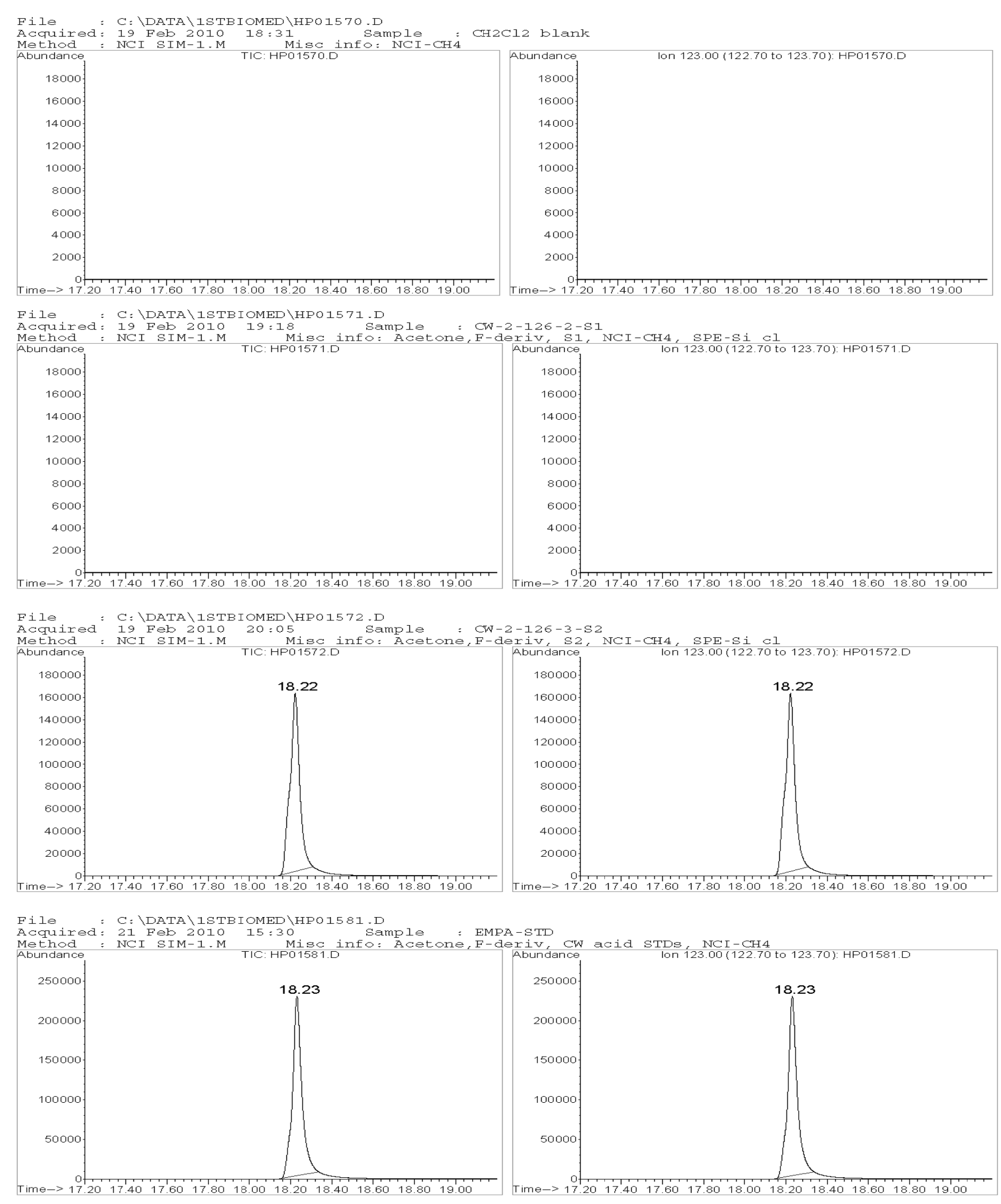

CI chromatograms supporting identification; TIC on left; EIC (m/z 123) on right.

Top: $\quad$ Chromatograms of solvent blank.

Second: Chromatograms of urine blank S1.

Third: $\quad$ Chromatograms of Urine sample S2, retention time $\mathbf{1 8 . 2 2}$ min.

Bottom: Chromatograms of reference standard of PFB derivative of Ethyl methylphosphonate retention time 18.23 min. 


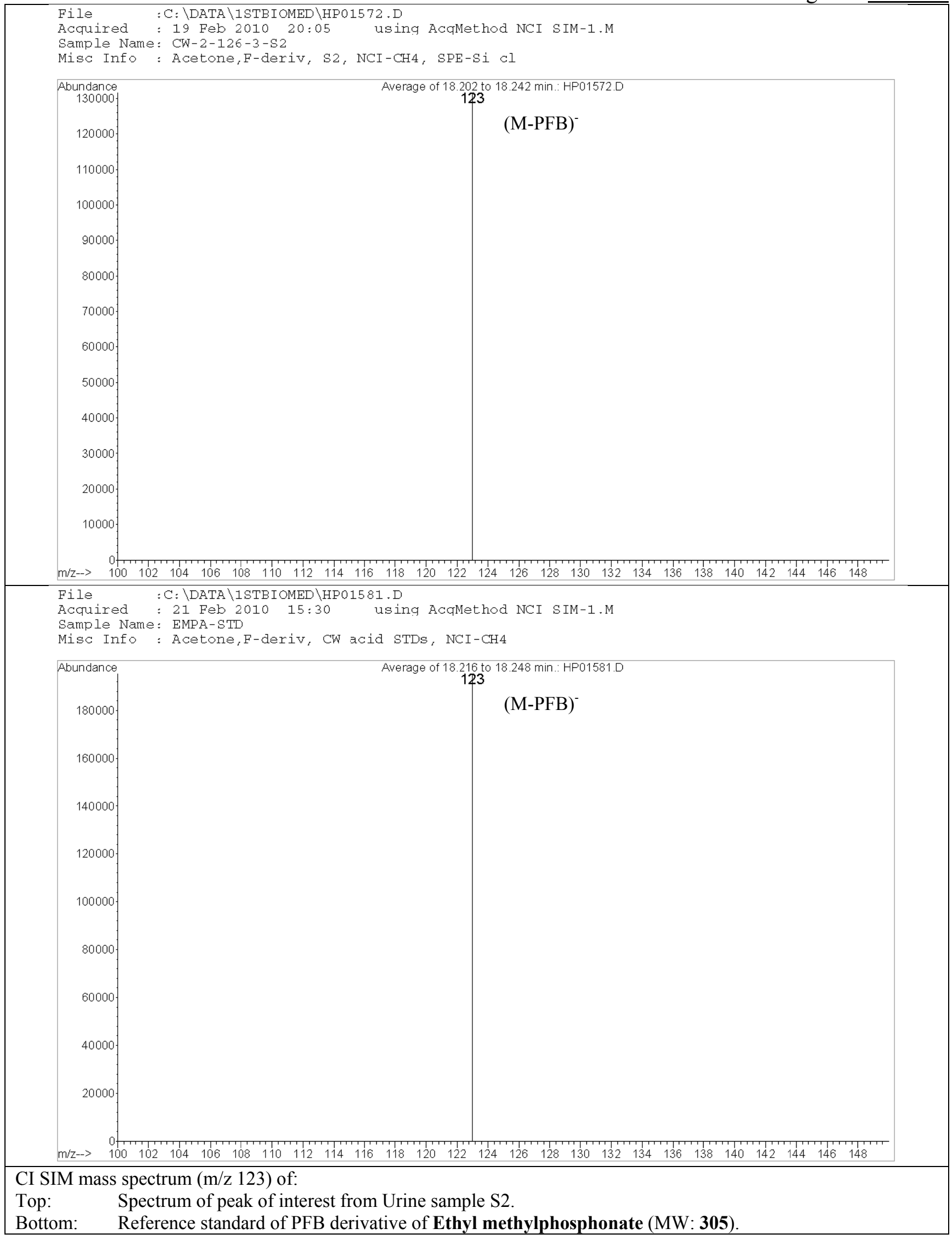


OPCW

C:IXcaliburldatalORBI000888_100221203952

Water blank

Page no.

15 Neg, MSMS, T3 column, 200uL/min

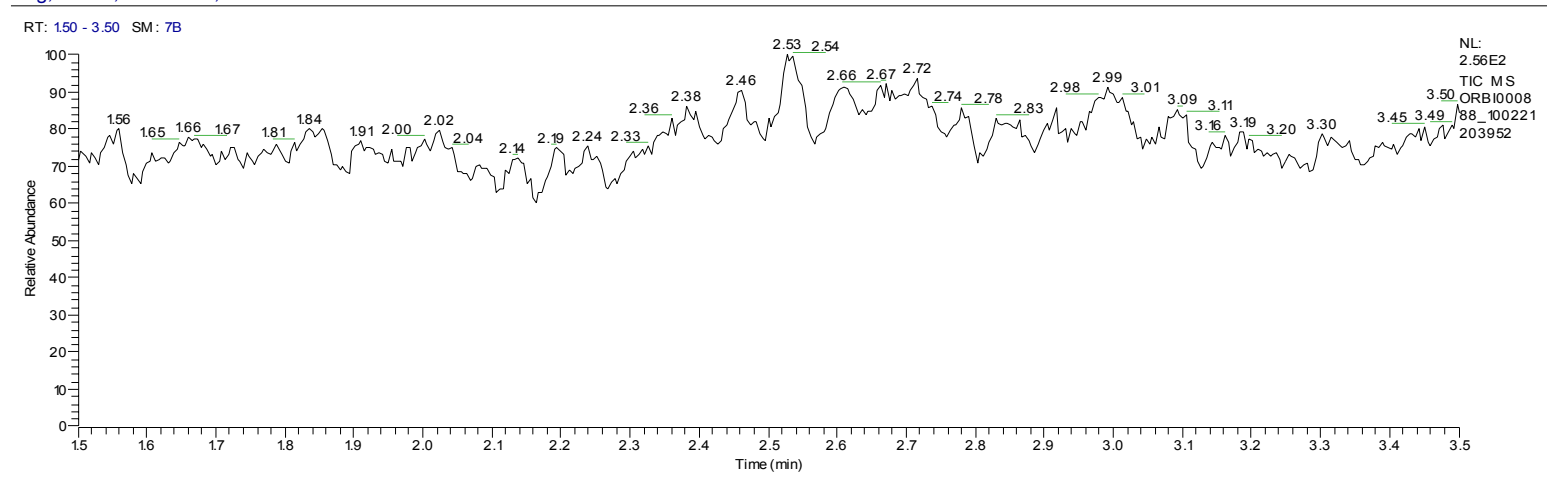

C:IXcaliburldatalORBI000889_100221210549 2/21/2010 9:05:49 PM CW-2-127-6-S

Neg, MSMS, T3 column, 200uL/min

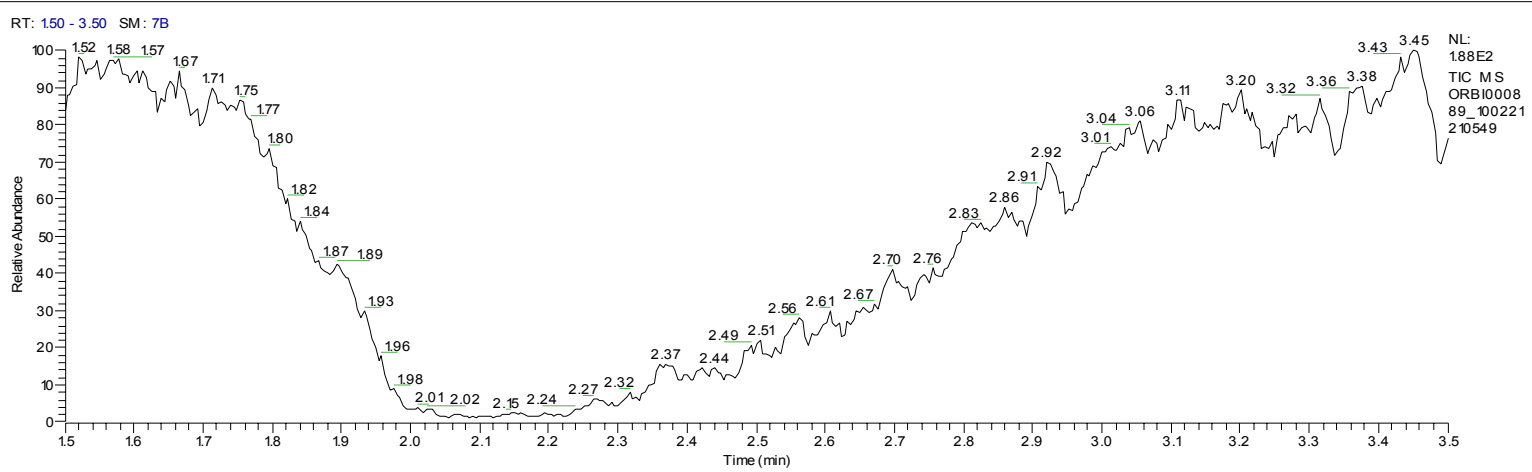

C:IXcaliburldatalORB 1000890

2/21/2010 9:31:45 PM CW-2-128-1S2

Neg, MSMS, T3 column, 200uL/min

RT: 1.50 - $3.50 \mathrm{SM}: 7 \mathrm{~B}$
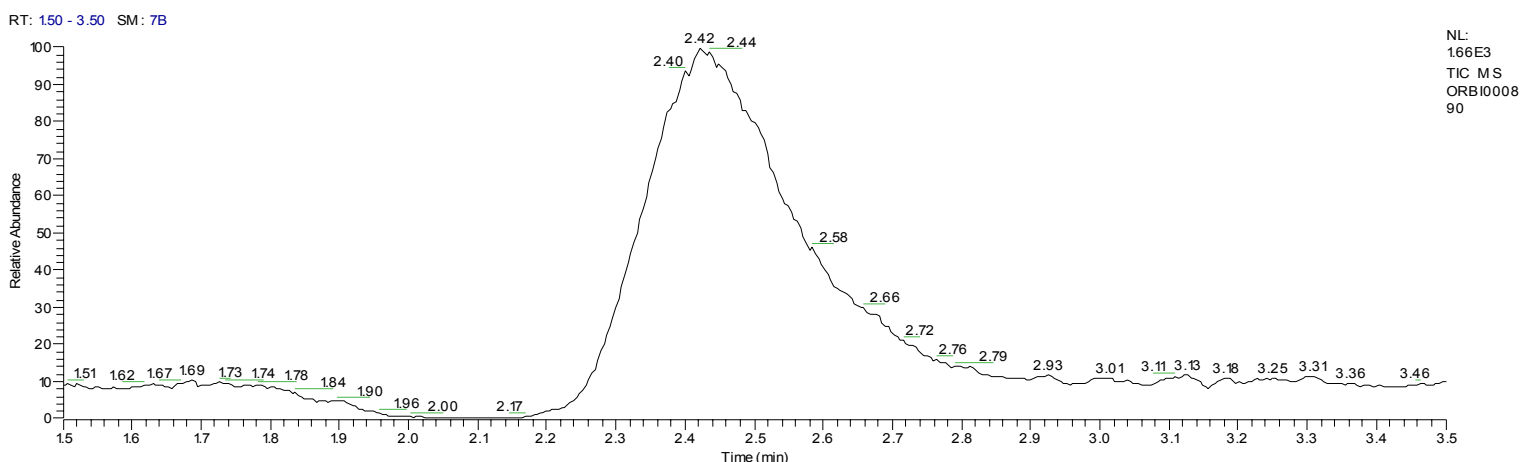

C.XCaliburIdatalORBI000891

Neg, MSMS, T3 column, 200uL/min

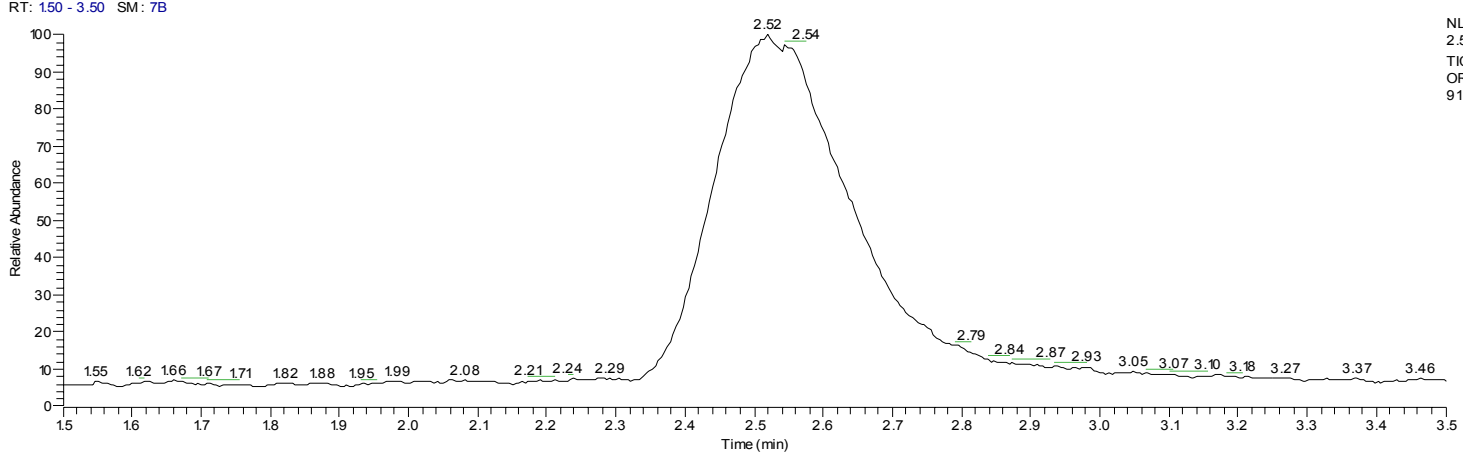

NL:

TIC MS

$\begin{array}{lllllllllll}2.76 & 2.79 & 2.93 & 3.01 & 3.11 & 3.13 & 3.18 & 3.25 & 3.31 & 3.36 & 3.46\end{array}$

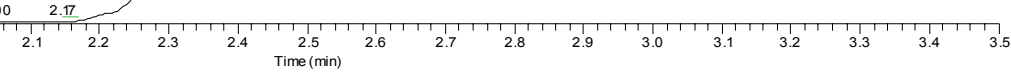

2/21/2010 9:57:41 PM

Cw-2-128-4-STD, EMPA

MS 
OPCW

C:IXcaliburldatalORBI000888_100221203952

Water blank

Page no.

(NL:

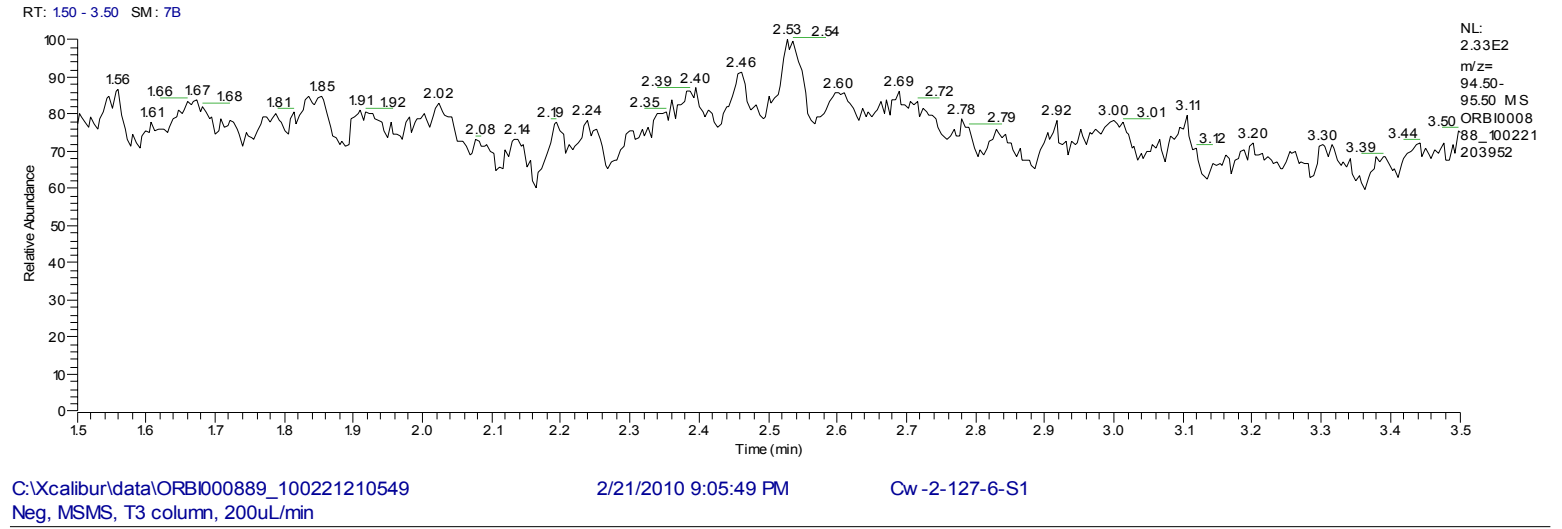

Neg, MSMS, T3 column, 200uL/min

RT: $1.50-3.50 \mathrm{SM}: 7 \mathrm{~B}$

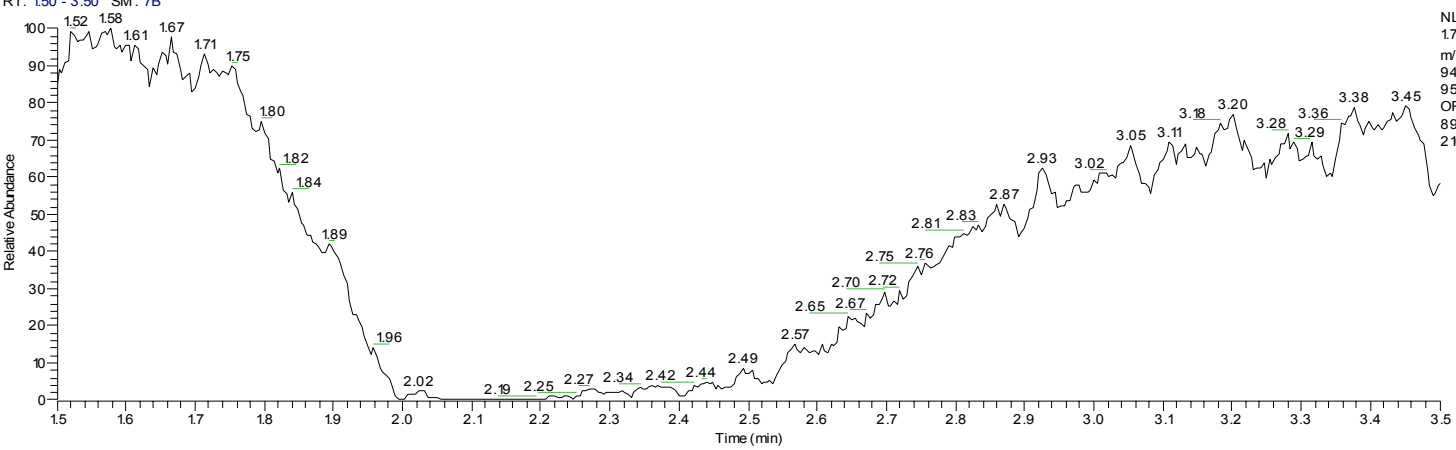

C:IXcaliburldatalORB 1000890

2/21/2010 9:31:45 PM

CW-2-128-1S2

Neg, MSMS, T3 column, 200uL/min

RT: 1.50 - 3.50 SM: $7 \mathrm{~B}$
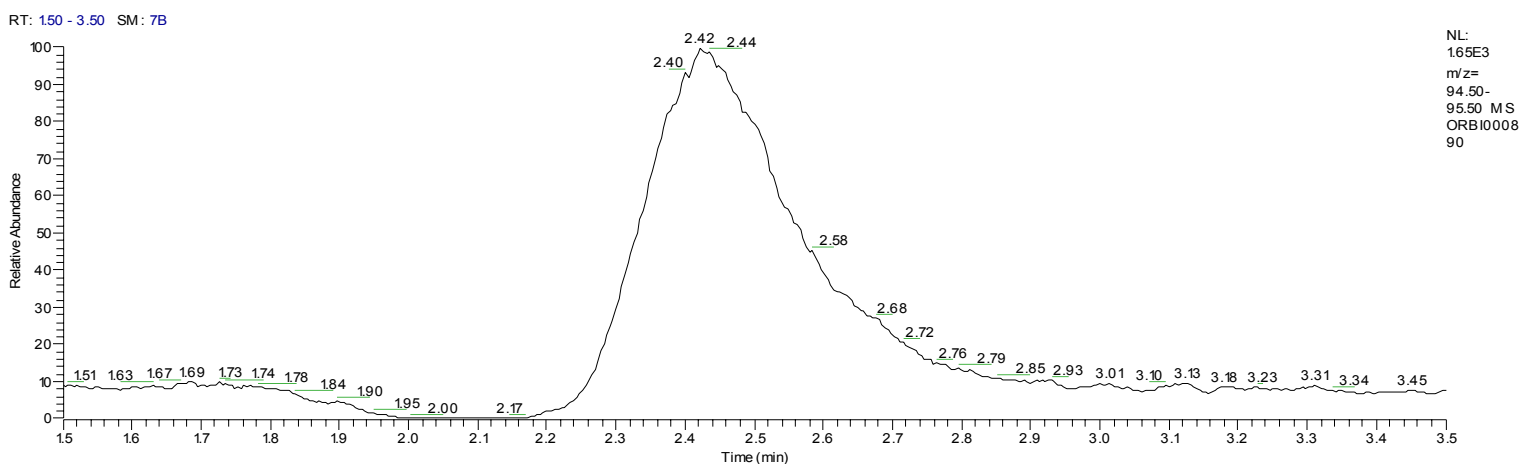

C:IXcaliburldatalORBI000891

Neg, MSMS, T3 column, 200uL/min

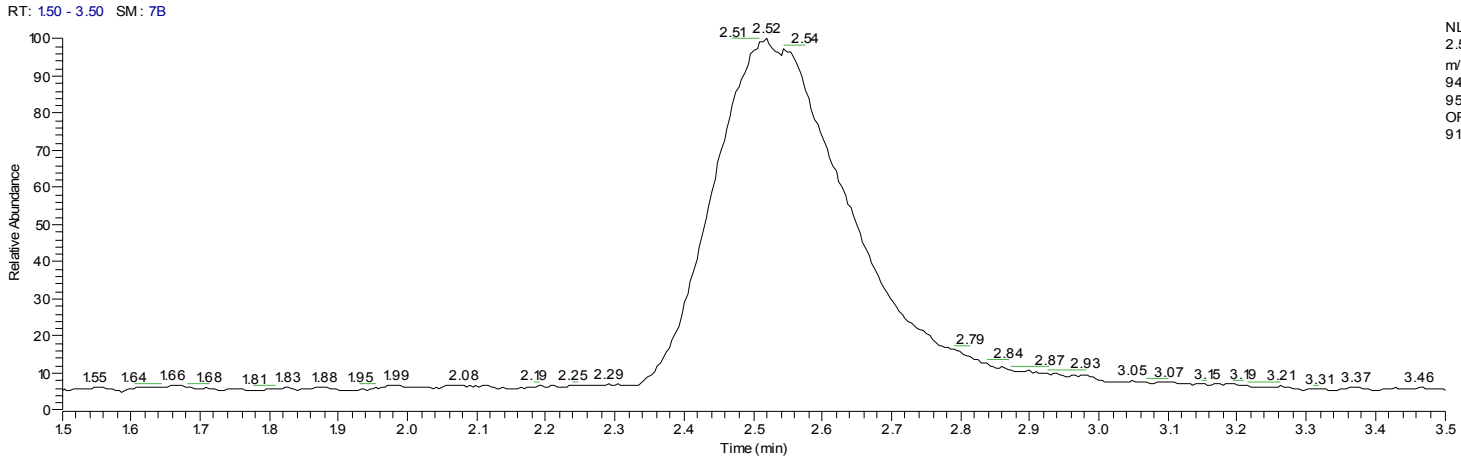

LC/MS/MS EIC (m/z 95) chromatograms. Top: water blank. Next: Urine blank S1. Third: Urine sample S2. Bottom: reference standard of Ethyl methylphosphonate 
OPCW

C:IXcaliburldatalORB1000890

Page no.

17

Neg, MSMS, T3 column, 200uL/min

ORBI000890 \#536-581 RT: 2.34-2.54 AV: $46 \quad$ NL: $1.37 E 3$

T: ITMS - c ESI Full ms2123.00@cid30.00 [50.00-210.00]

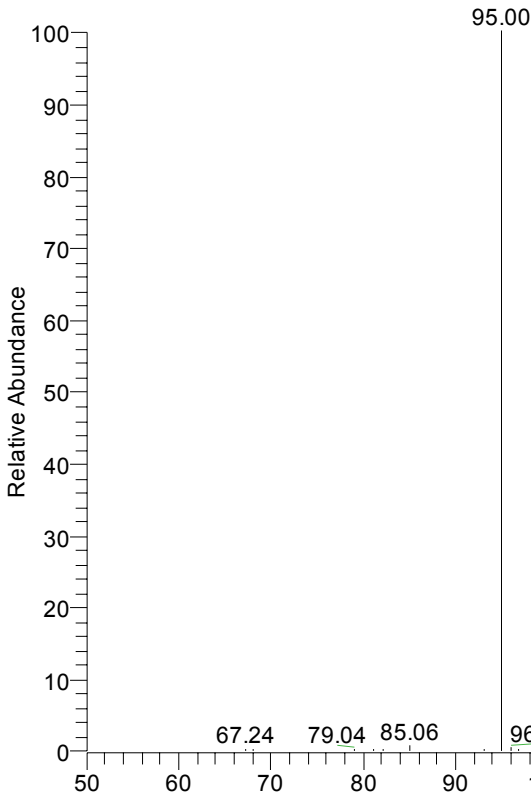

\begin{tabular}{|c|c|c|c|c|}
\hline 140 & 150 & 160 & 170 & 180 \\
\hline
\end{tabular}

C:IXcaliburldatalORBI000891 Neg, MSMS, T3 column, 200uL/min

Cw-2-128-4-STD, EMPA

ORBI000891 \#562-595 RT: 2.46-2.60 AV: 34 NL: $2.28 E 3$

T: ITMS - c ESI Full ms2123.00@cid30.00 [50.00-210.00]

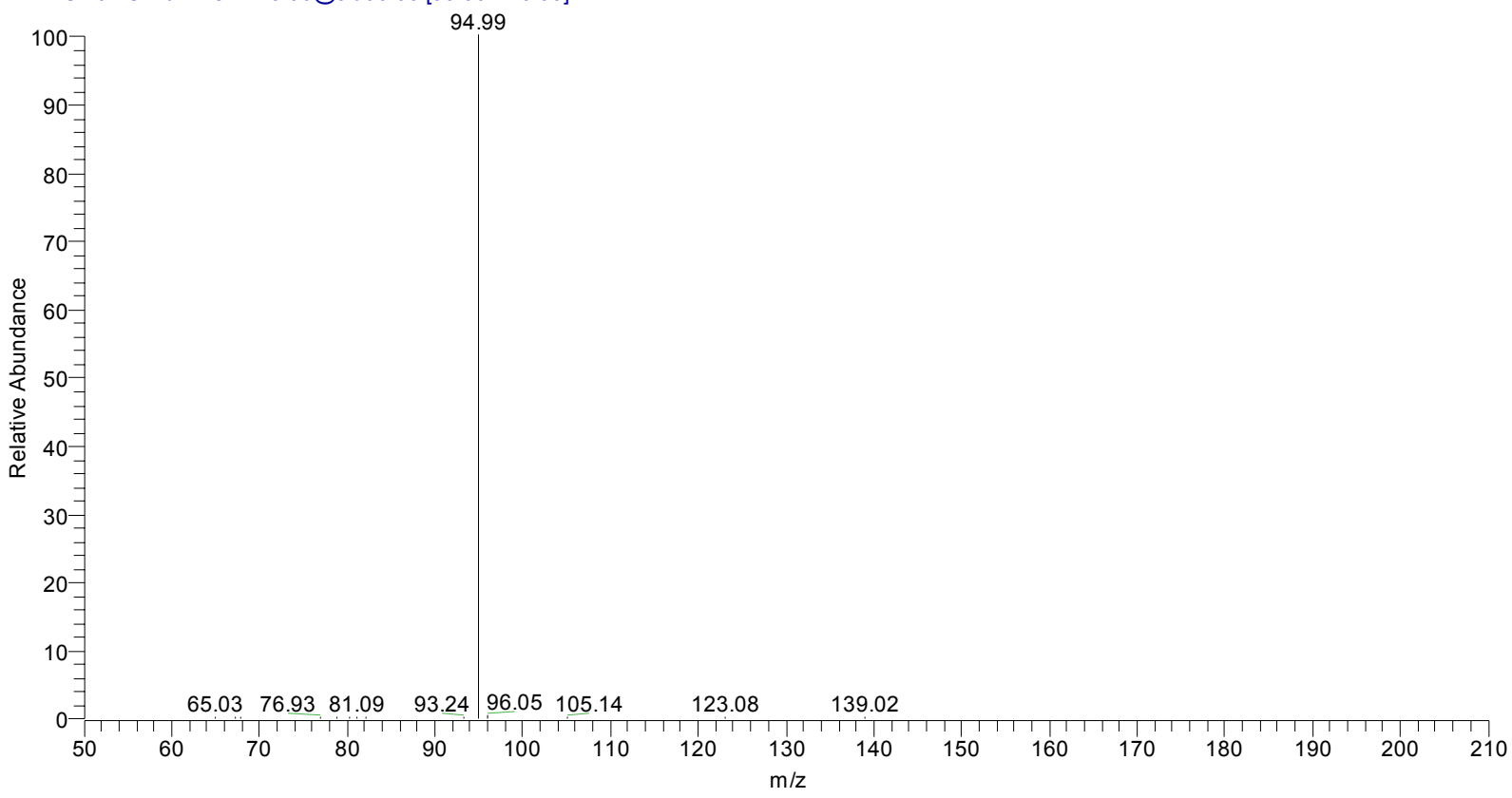

LC/MS/MS spectra - product spectra of parent $[\mathrm{M}-\mathrm{H}]^{+-} \mathrm{m} / \mathrm{z}$ 123. Top: Urine sample S2. Bottom: reference standard of Ethyl methylphosphonate 


\section{RESULTS: URINE SAMPLE S3}

$\beta$-Lyase metabolites of sulfur mustard were not found in this sample (after sample preparation that included $\mathrm{TiCl}_{3}$ reduction and analysis by GC-GC-MS (EI)).

Isopropyl methyphosophonate was found. The following few pages present the GC-MS (CI) data for the derivative and the LC-MS-MS data for the un-derivitized acid. 


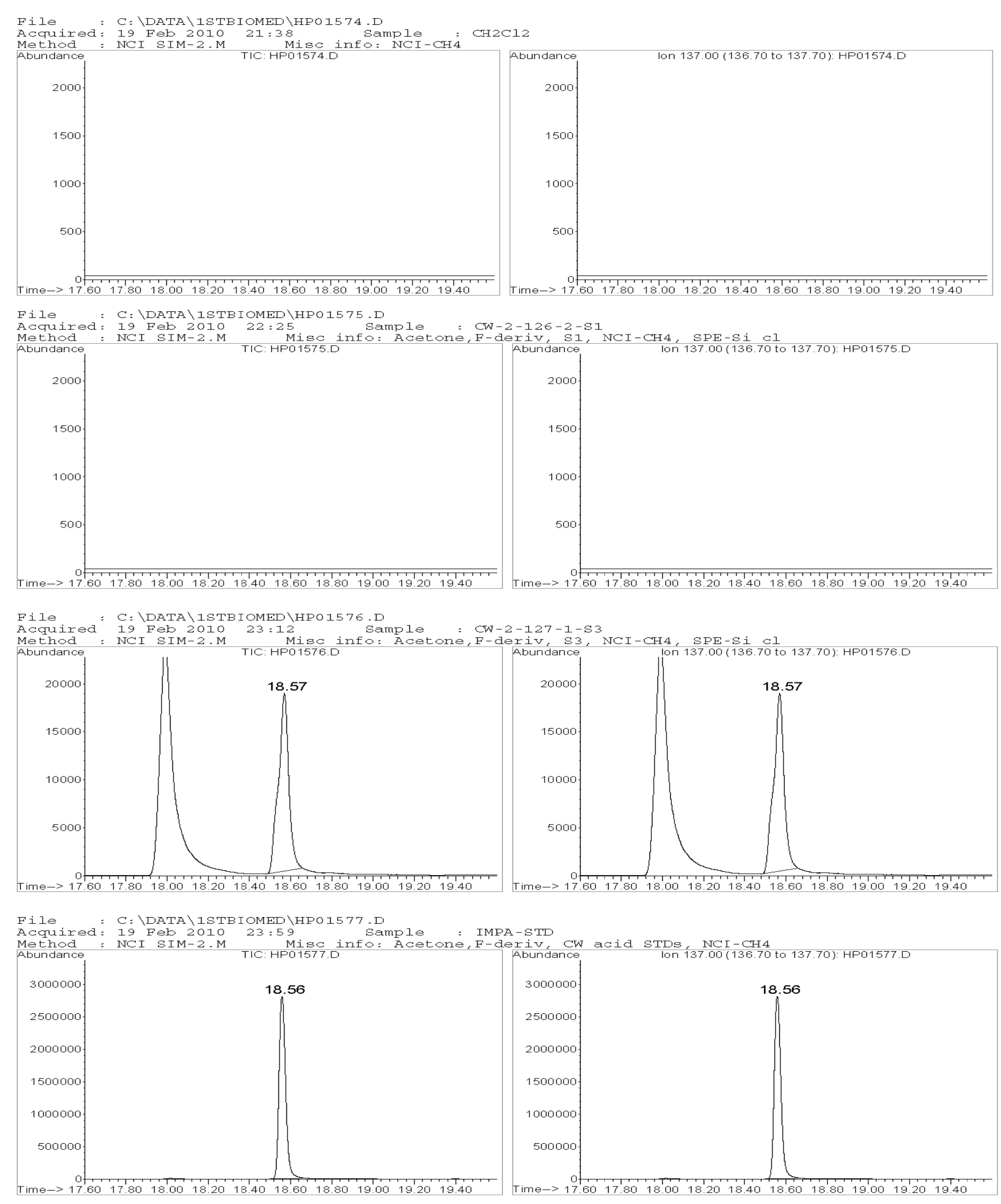

CI chromatograms supporting identification; TIC on left; EIC (m/z 137) on right.

Top: $\quad$ Chromatograms of solvent blank.

Second: Chromatograms of urine blank S1.

Third: $\quad$ Chromatograms of Urine sample S3, retention time $\mathbf{1 8 . 5 7}$ min.

Bottom: Chromatograms of reference standard of PFB derivative of Isopropyl methylphosphonate retention time $18.56 \mathrm{~min}$. 
File :C: \DATA \1STBIOMED $\backslash$ HPO 1576.D

Acquired : 19 Feb 2010 23:12 using AcqMethod NCI SIM-2.M

Sample Name: CW-2-127-1-S3

Misc Info: Acetone,F-deriv, S3, NCI-CH4, SPE-Si Cl

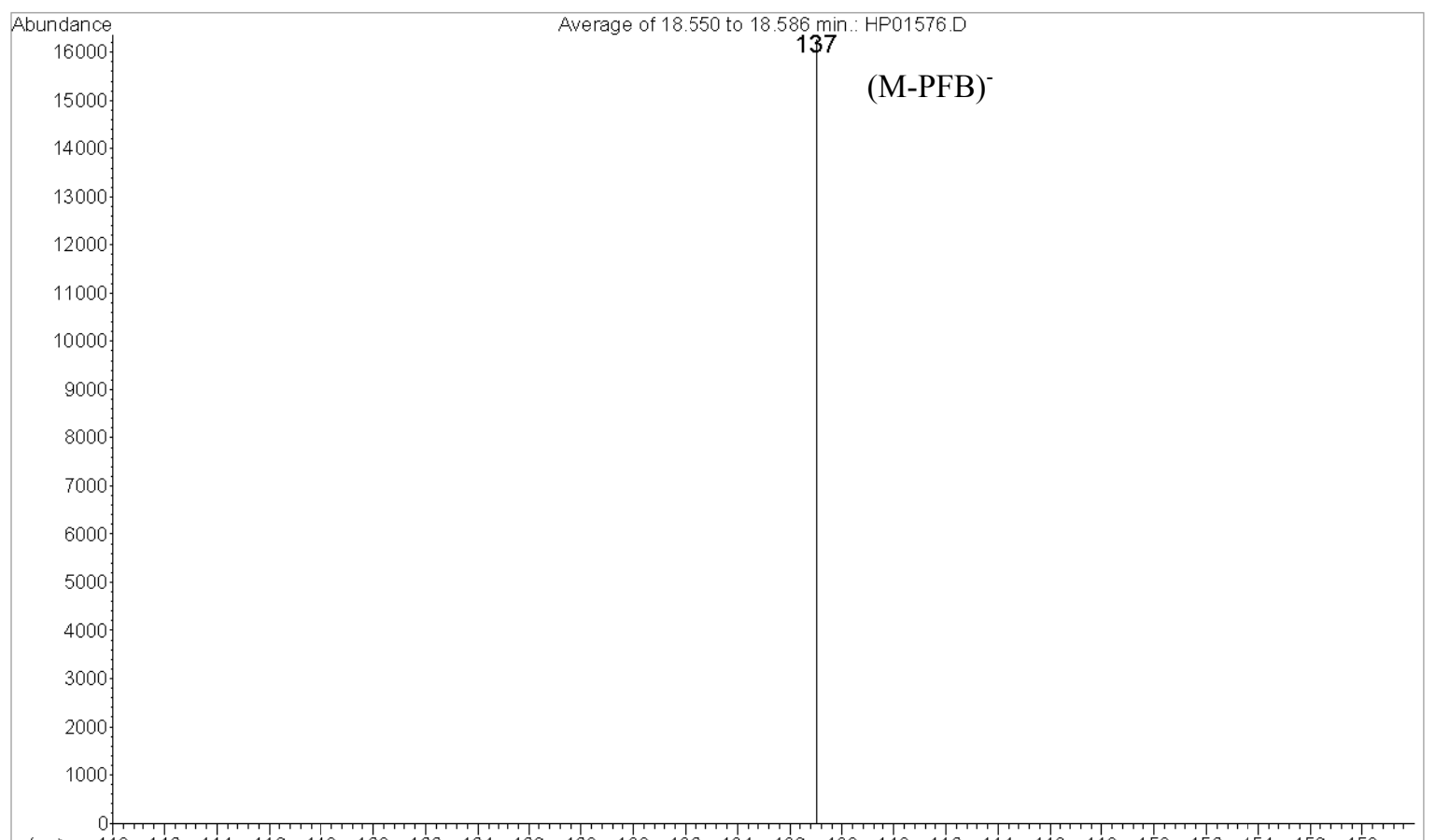

m/z--> $\begin{array}{llllllllllllllllllllllllll}110 & 112 & 114 & 116 & 118 & 120 & 122 & 124 & 126 & 128 & 130 & 132 & 134 & 136 & 138 & 140 & 142 & 144 & 146 & 148 & 150 & 152 & 154 & 156 & 158\end{array}$

File $\quad$ C: $\backslash$ DATA $\backslash$ 1STBIOMED $\backslash H P 01577 . D$

Acquired : 19 Feb 2010 23:59 using AcqMethod NCI SIM-2.M

Sample Name: IMPA-STD

Misc Info: Acetone,F-deriv, CW acid STDs, NCI-CH4

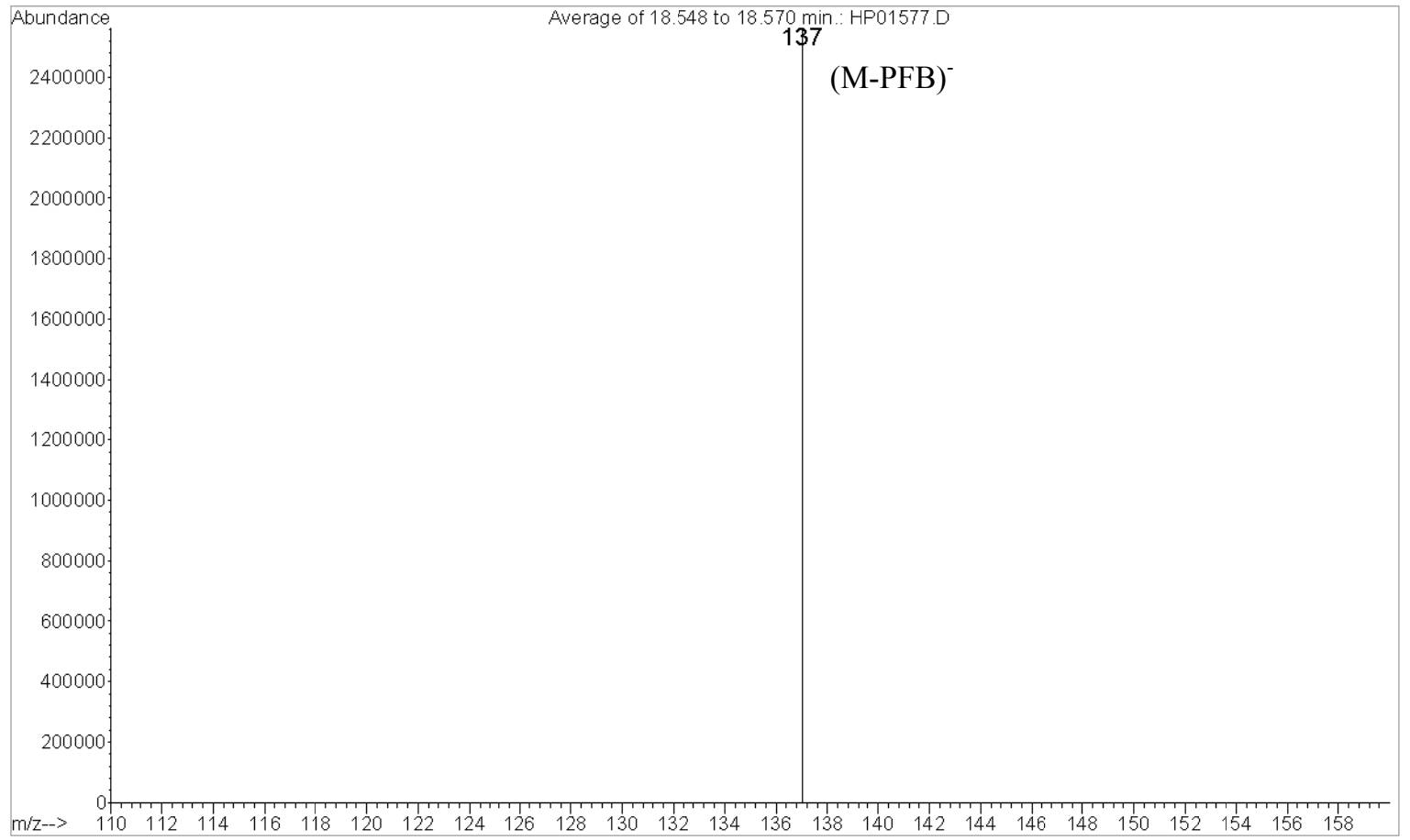

CI SIM mass spectrum (m/z 137) of:

Top: $\quad$ Spectrum of peak of interest from Urine sample S3.

Bottom: $\quad$ Reference standard of PFB derivative of Isopropyl methylphosphonate (MW: 319). 
OPCW

C:IXcaliburldatalORBI000892_100221224934

Neg, MSMS, T3 column, 200uL/min

Page no.

RT: $1.70-3.70 \mathrm{SM}: 7 \mathrm{~B}$

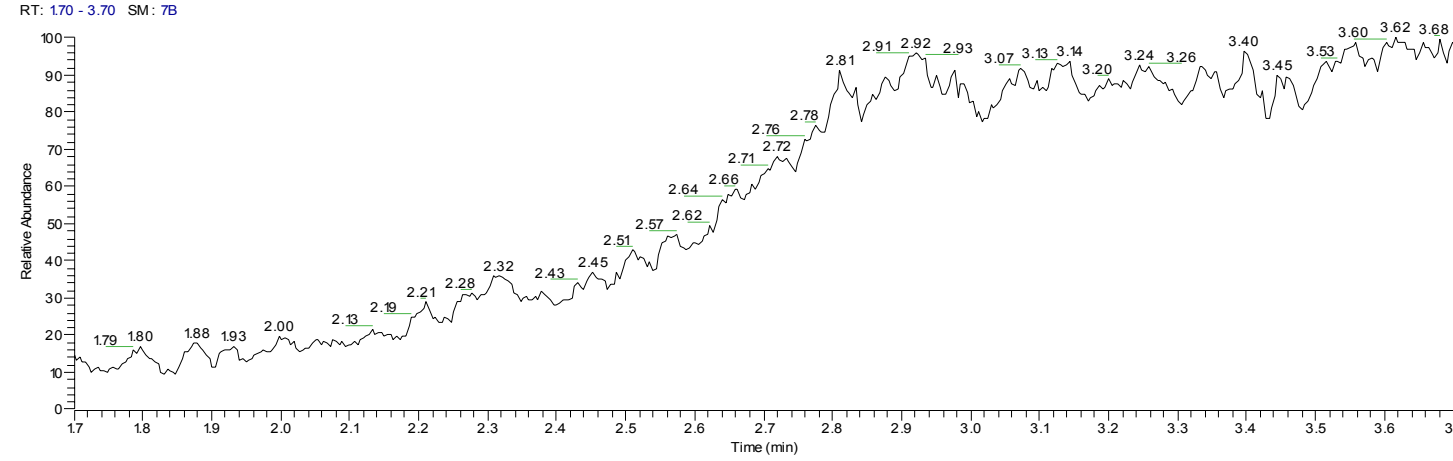

C:IXcaliburldatalORBI000893

2/21/2010 11:15:30 PM CW-2-127-6-S1

Neg, MSMS, T3 column, 200uL/min

RT: $1.70-3.70 \mathrm{SM}: 7 \mathrm{~B}$

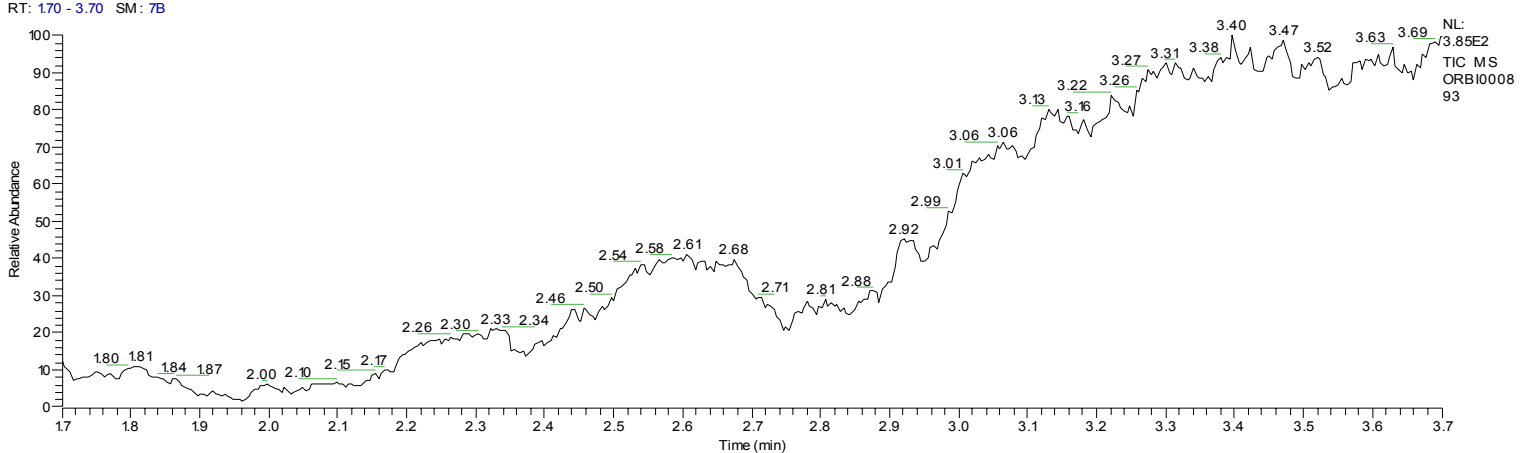

C:IXcaliburldatalORBI000894

2/21/2010 11:41:27 PM CW-2-128-2-S3

Neg, MSMS, T3 column, 200uL/min

RT: 1.70 - $3.70 \mathrm{SM}: 7 \mathrm{~B}$

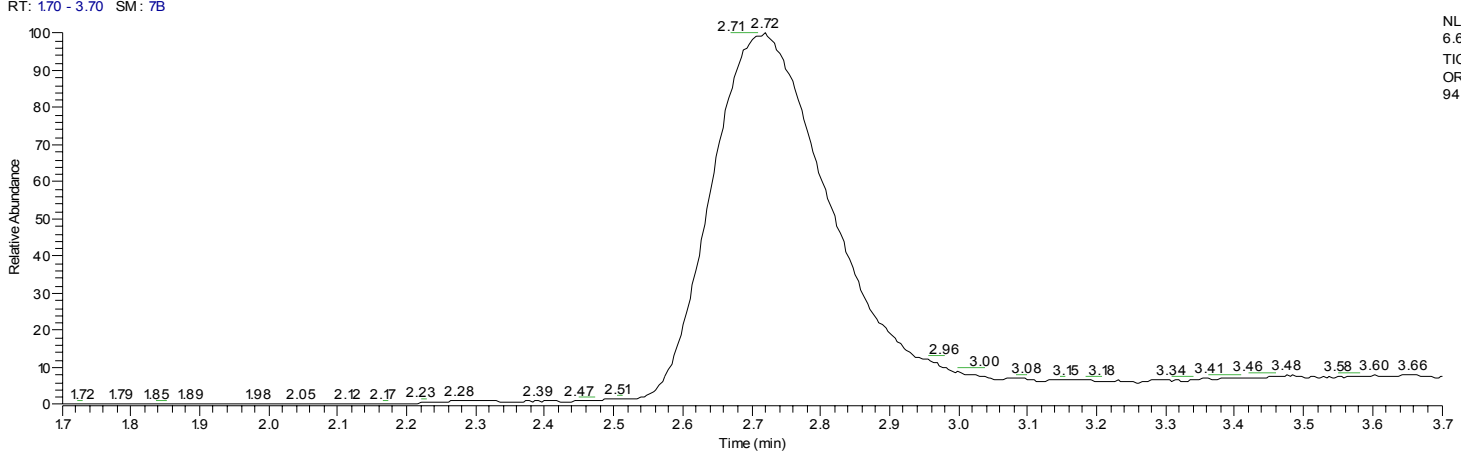

C:IXcaliburldatalORBI000895

Neg, MSMS, T3 column, 200uL/min

2/22/2010 12:07:23 AM CW-2-128-5-STD, IMPA

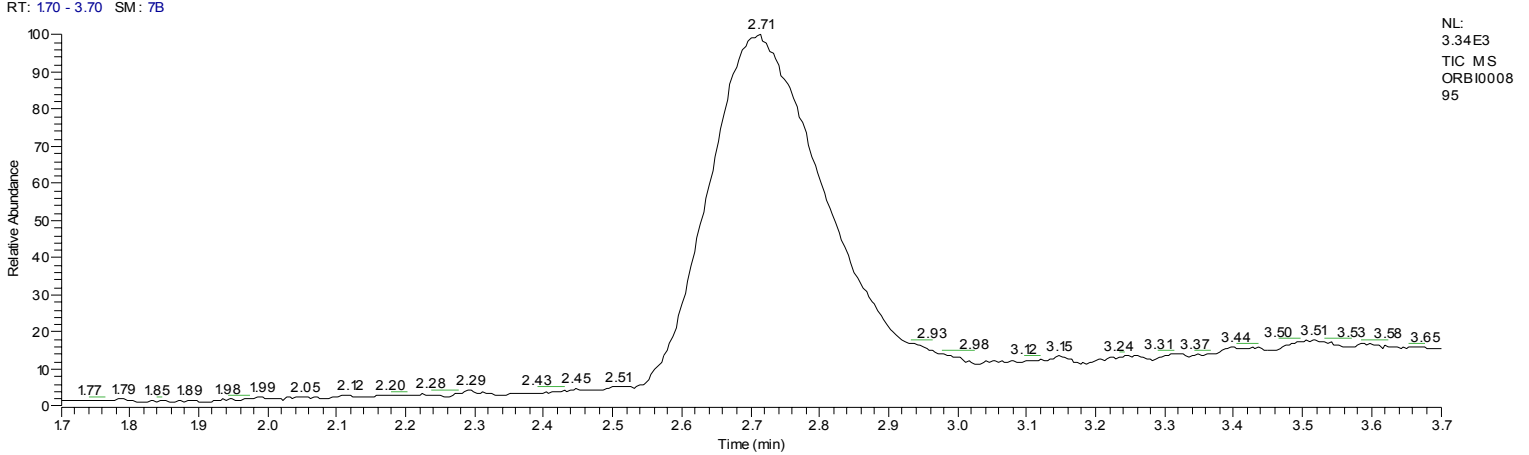

LC/MS/MS chromatograms. Top: water blank. Next: Urine blank S1. Third: Urine sample S3. Bottom: reference standard of Isopropyl methylphosphonate 


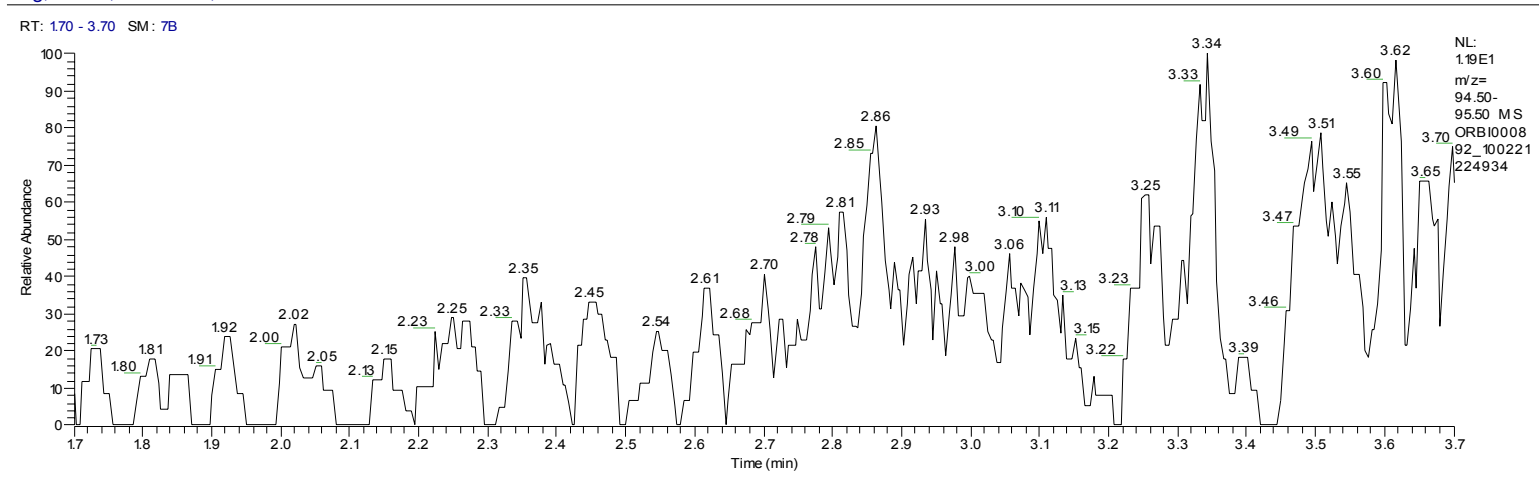

C:IXcaliburldatalORBI000893

2/21/2010 11:15:30 PM CW-2-127-6-S1

Neg, MSMS, T3 column, 200uL/min

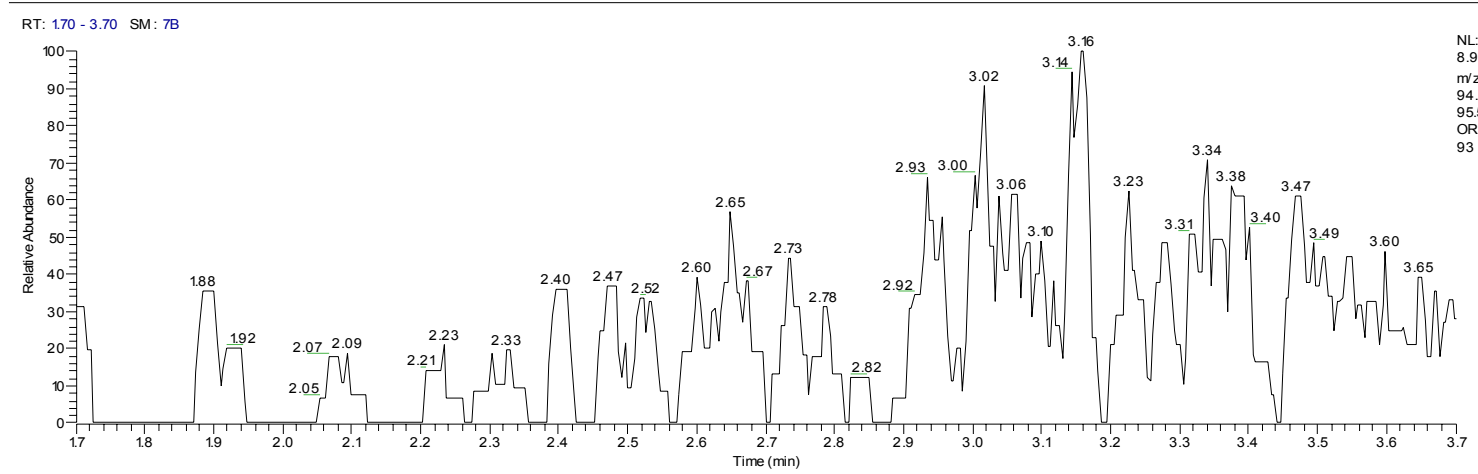

C:IXcaliburldatalORBI000894

2/21/2010 11:41:27 PM CW-2-128-2-S3

Neg, MSMS, T3 column, 200uL/min

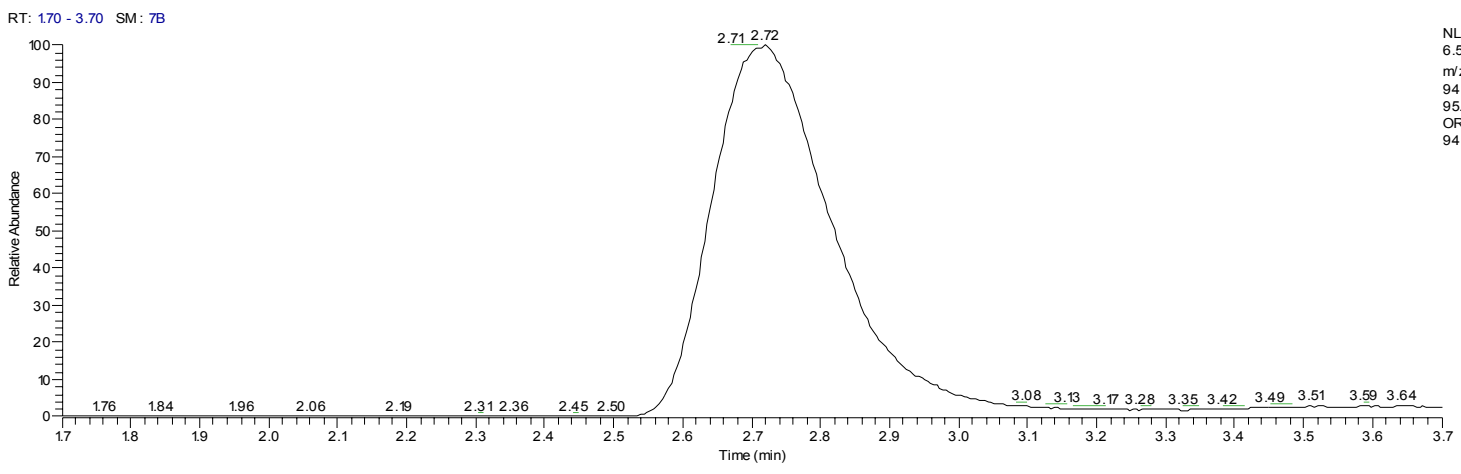

C:IXcaliburldatalORBI000895

Neg, MSMS, T3 column, 200uL/min

2/22/2010 12:07:23 AM CW-2-128-5-STD, IMPA

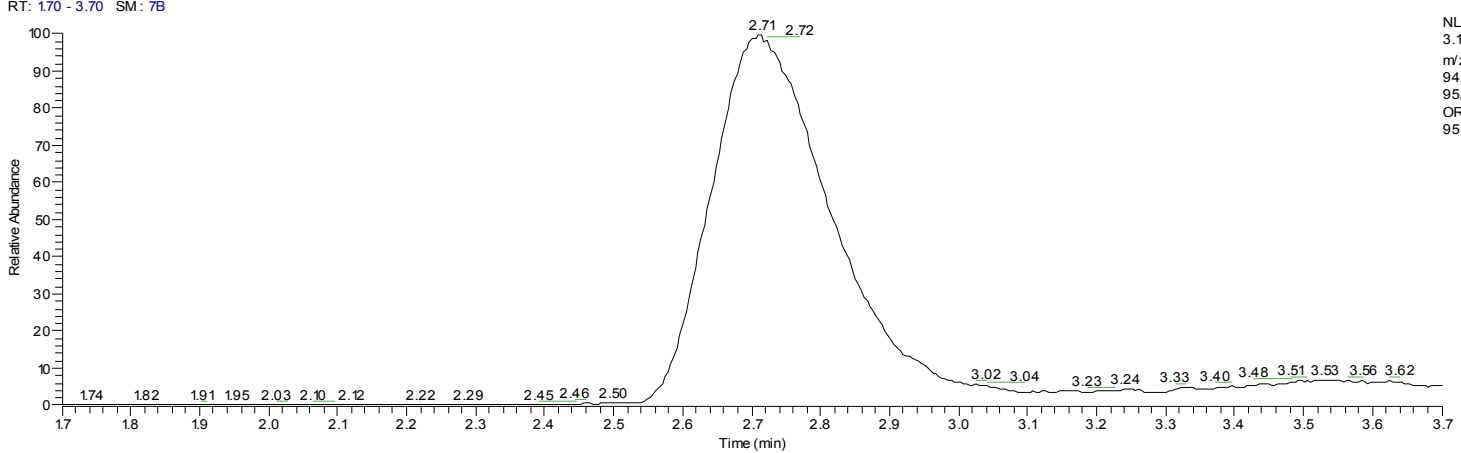

LC/MS/MS EIC (m/z 95) chromatograms. Top: water blank. Next: Urine blank S1. Third: Urine sample S3. Bottom: reference standard of Isopropyl methylphosphonate 
OPCW

C:IXcaliburldatalORB1000894

Neg, MSMS, T3 column, 200uL/min

ORBI000894 \#605-639 RT: 2.64-2.79 AV: $35 \quad$ NL: $5.69 E 3$

T: ITMS - c ESI Full ms2137.00@cid33.00 [50.00-210.00]

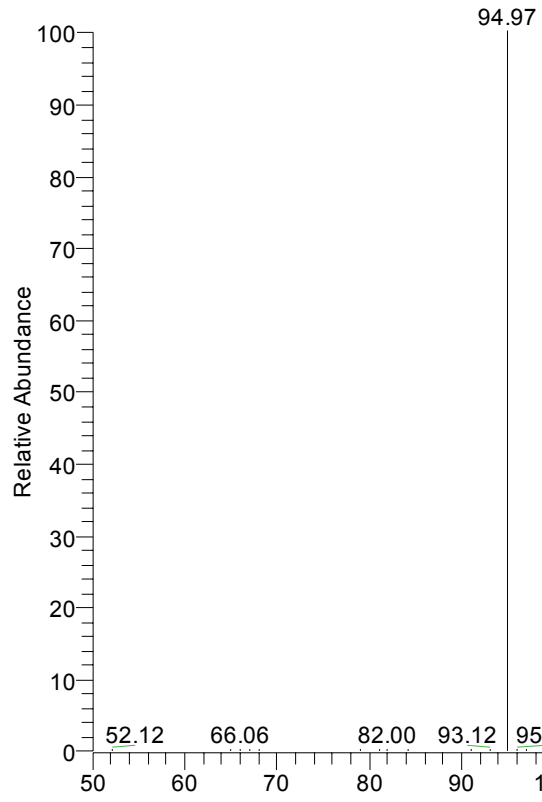

C:IXcaliburldatalORBI000895 Neg, MSMS, T3 column, 200uL/min

ORBI000895 \#606-637 RT: 2.65-2.78 AV: 32 NL: $2.78 E 3$

T: ITMS - c ESI Full ms2 137.00@cid33.00 [50.00-210.00]

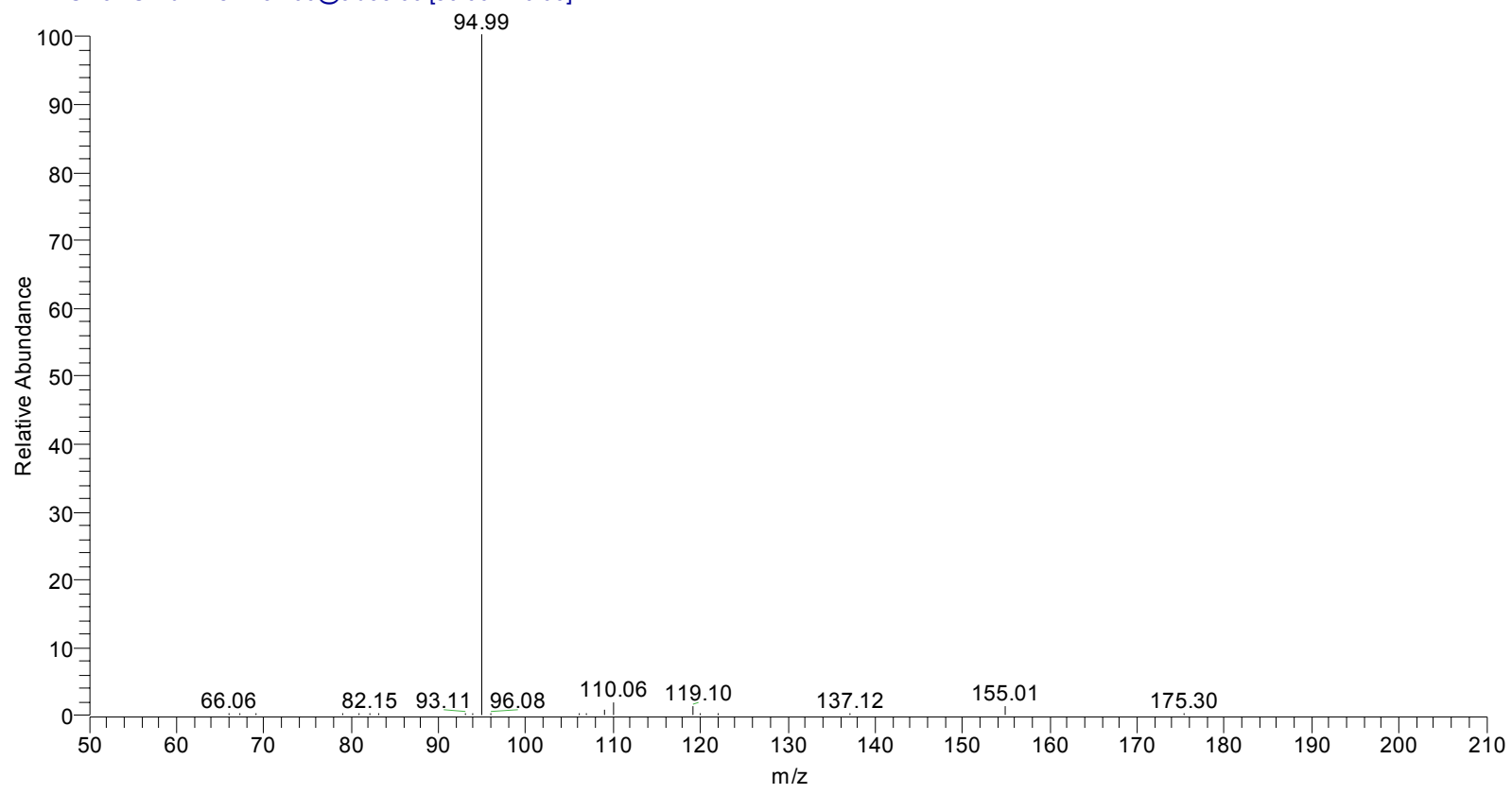

LC/MS/MS spectra - product spectra of parent $[\mathrm{M}-\mathrm{H}]^{-} \mathrm{m} / \mathrm{z}$ 137. Top: Urine sample S3. Bottom: reference standard of Isopropyl methylphosphonate 


\section{RESULTS: URINE SAMPLE S4}

$\beta$-Lyase metabolites of sulfur mustard were not found in this sample (after sample preparation that included $\mathrm{TiCl}_{3}$ reduction and analysis by GC-GC-MS (EI)).

None of the phosphonic acids were found. 


\section{RESULTS: URINE SAMPLE S5}

A $\beta$-Lyase metabolite of sulfur mustard was found in this sample (after sample preparation that included $\mathrm{TiCl}_{3}$ reduction and analysis by GC-GC-MS (EI)) and confirmed with GC-MS (CI) and LC-MS-MS.

Present was 1,1'-Sulfonylbis[2-(methylsulfinyl)ethane] (SBMSE), but no evidence of 1Methylsulfinyl-2-[2-(methylthio)ethylsulfonyl]ethane (MSMTESE) was observed.

None of the phosphonic acids were found.

The following few pages present the GC-GC-MS (EI) and GC-MS (CI) data for the derivative and the LC-MS-MS data for the un-derivitized metabolite. 
File $\quad$ : C:LLNL2010`TOF71

Acquired: 14 Jan 2009 2:35:45 PM Sample: CH2Cl2 Blank Lot\# 7284M
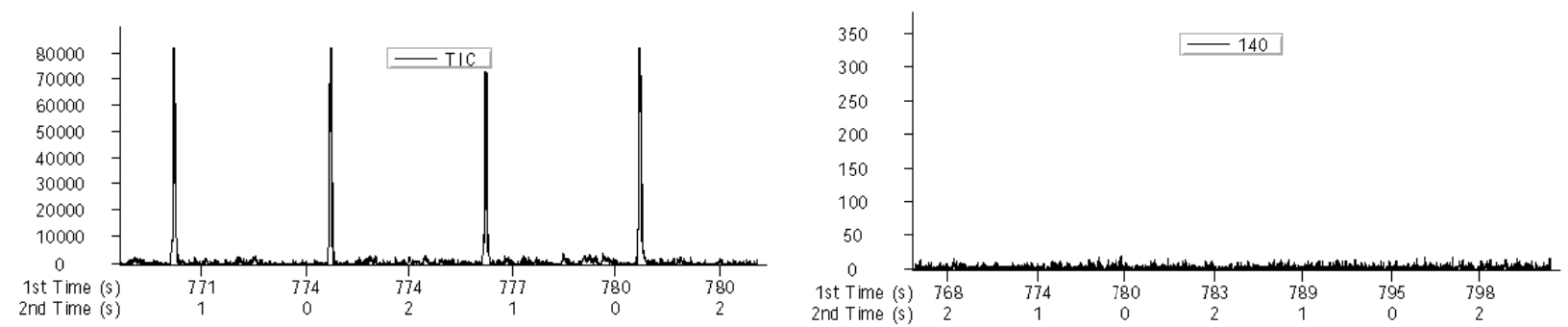

File : C:LLNL2010 \TOF72

Acquired: 14 Jan 2009 3:02:50 PM Sample: CW-2-122-3-S1
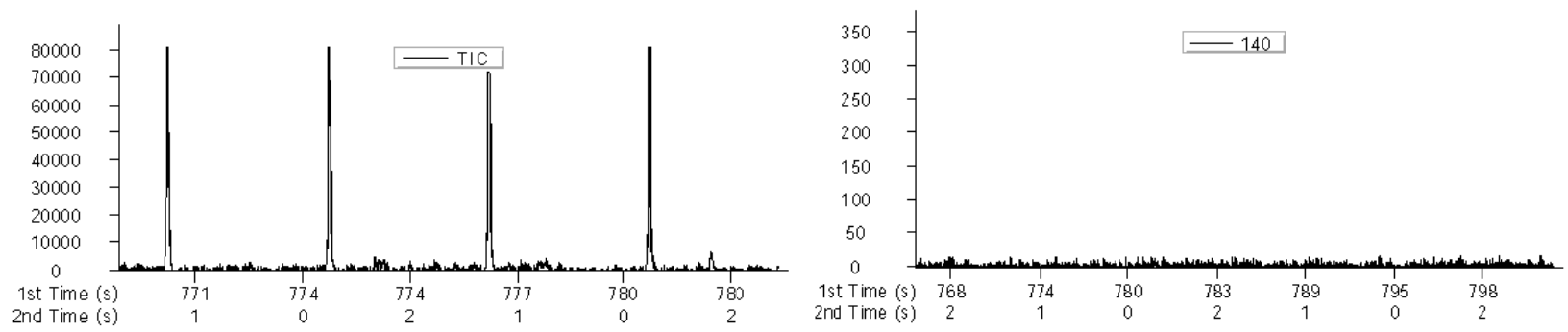

File : C:LLNL2010 1 TOF73

Acquired: 14 Jan 2009 3:39:35 PM Sample: CW-2-123-1-S5
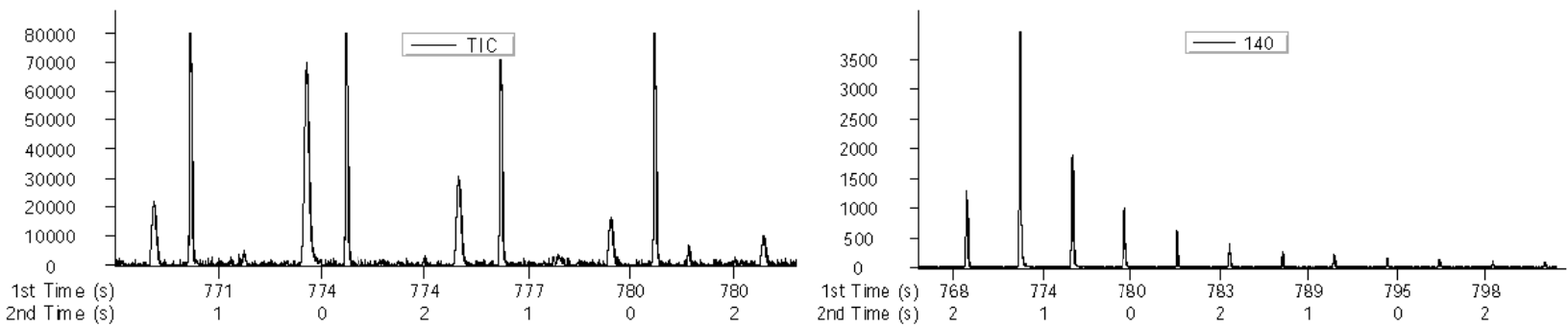

File $\quad$ : C:LLNL2010 \TOF74

Acquired: 14 Jan 2009 4:16:16 PM Sample: 0.5 ppm SBMTE
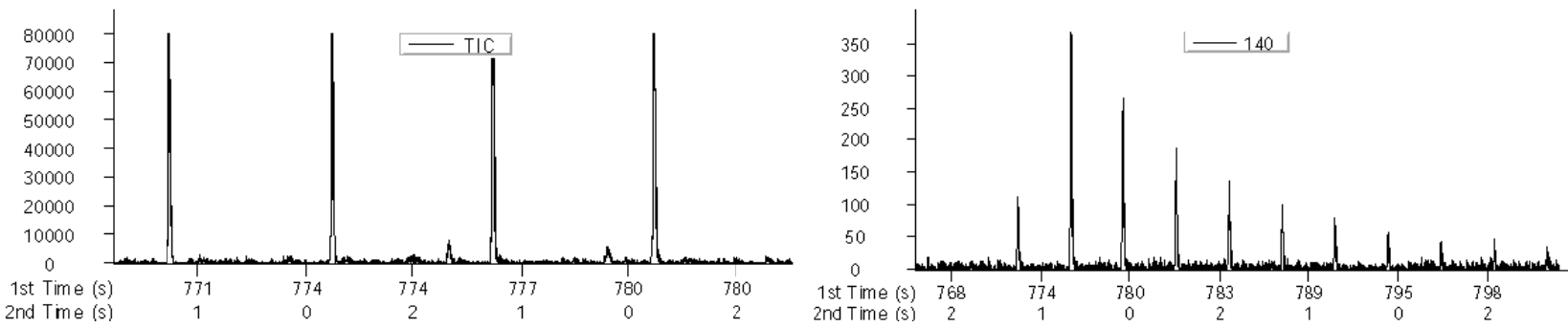

EI chromatograms supporting identification; TIC on left; EIC (m/z 140) on right

Top: Chromatograms of solvent blank.

Second: Chromatograms of urine blank S1.

Third: Chromatograms of urine sample S5, retention time 771, $2.710 \mathrm{sec}$

Bottom: Chromatograms of reference standard of 1,1'-Sulfonylbis[2-9methylthio)ethane], retention time 774, 2.660 sec 


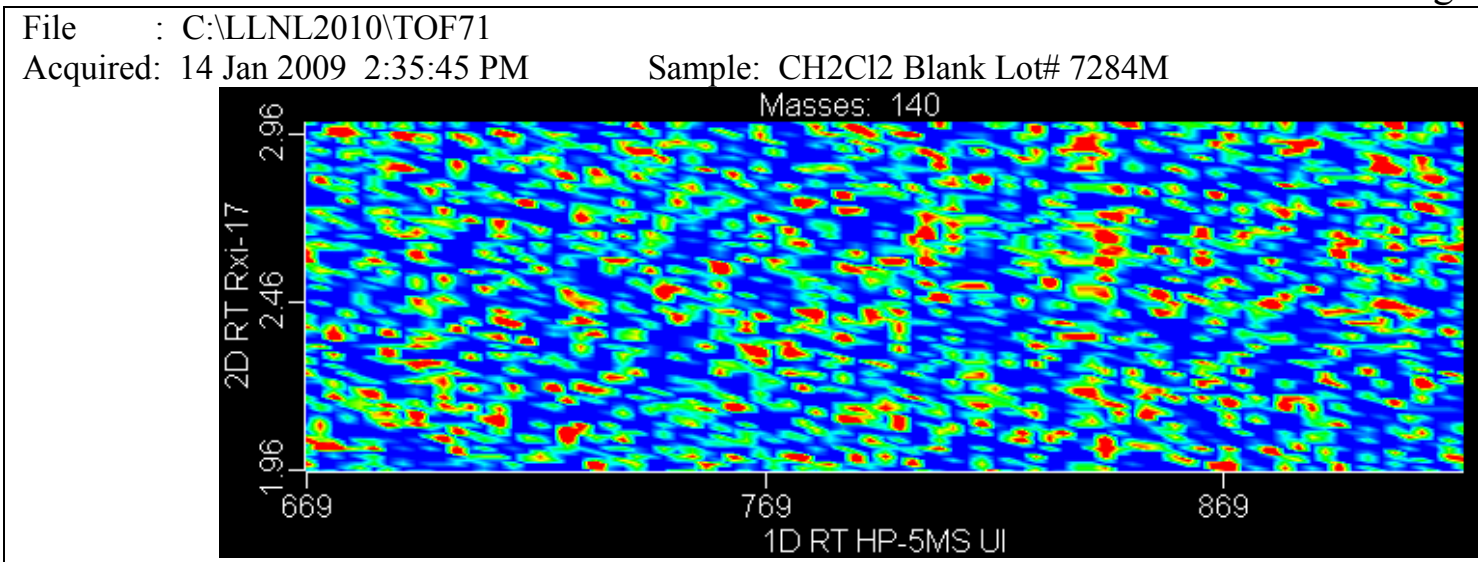

File $\quad$ : C:LLLN2010\TOF72

Acquired: 14 Jan 2009 3:02:50 PM Sample: CW-2-122-3-S1

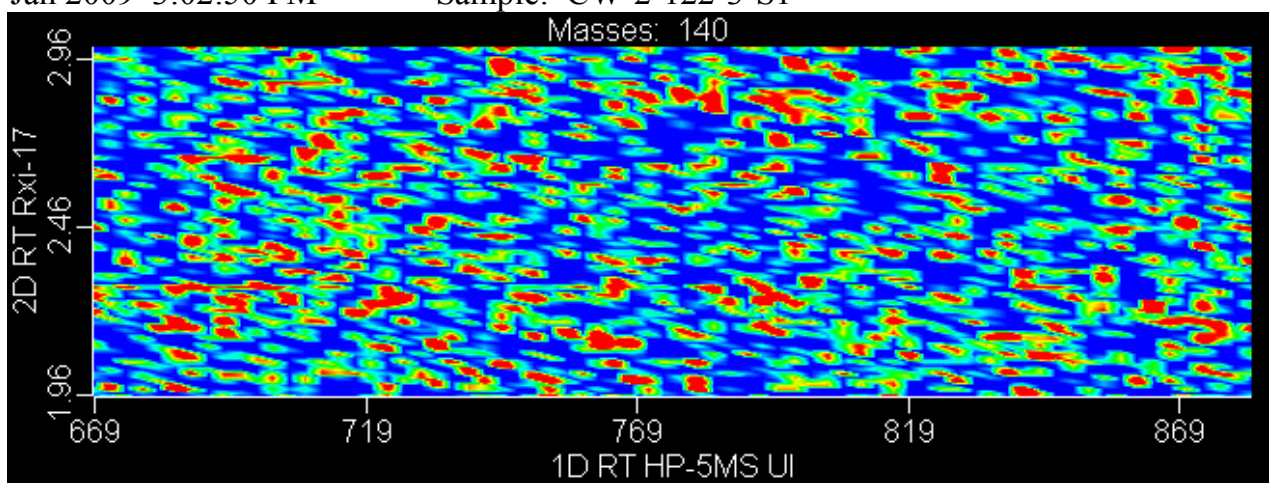

File : C:ILLNL2010\TOF73

Acquired: 14 Jan 2009 3:39:35 PM

Sample: CW-2-123-1-S5

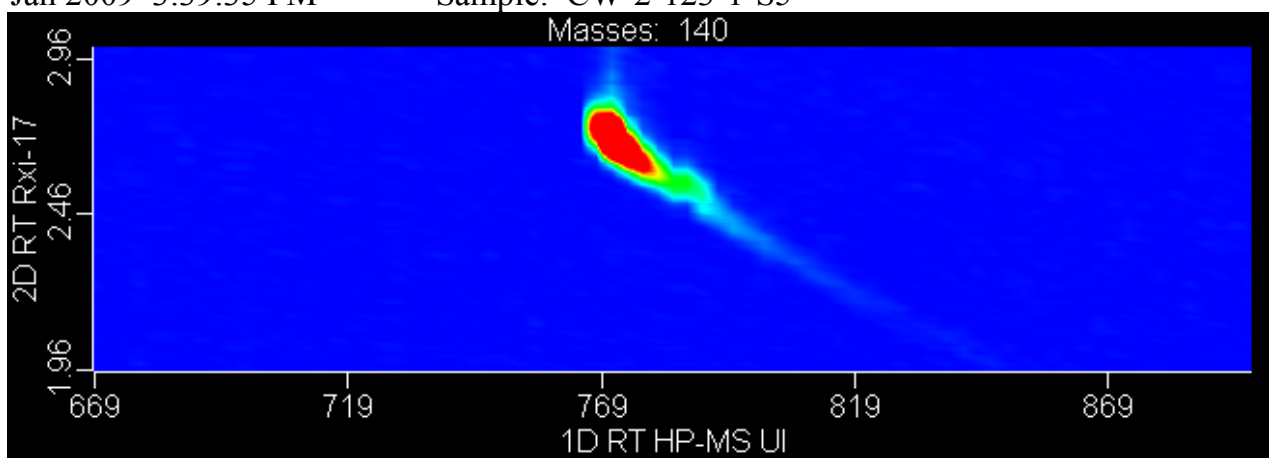

File $\quad$ : C: LLNL2010\TOF74

Acquired: 14 Jan 2009 4:16:16 PM

Sample: $0.5 \mathrm{ppm}$ SBMTE

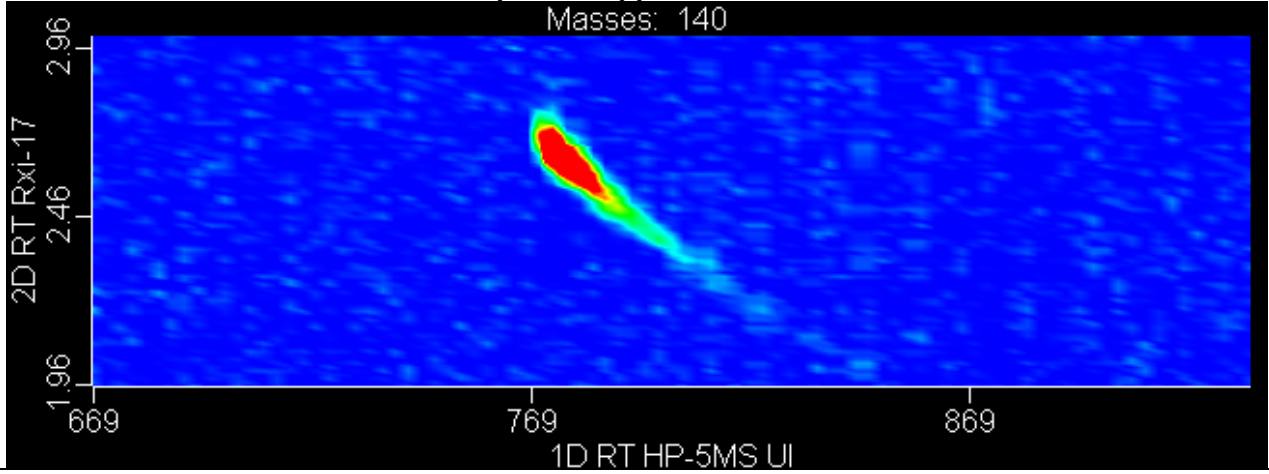

2D Contour plot supporting identification; EIC (m/z 140)

Top: Contour plot of solvent blank; Second: Contour plot of urine blank S1; Third: Contour plot of urine sample S5, retention time 771, $2.710 \mathrm{sec}$; Bottom:Contour plot of reference standard of 1,1'-Sulfonylbis[2-9methylthio)ethane], retention time $774,2.660 \mathrm{sec}$ 
File : C:\LLNL2010\TOF73

Acquired: 14 Jan 2009 3:39:35 PM

Sample: CW-2-123-1-S5

Peak True - sample "TOF:73", peak 185, at 771 , $2.710 \mathrm{sec}$, sec

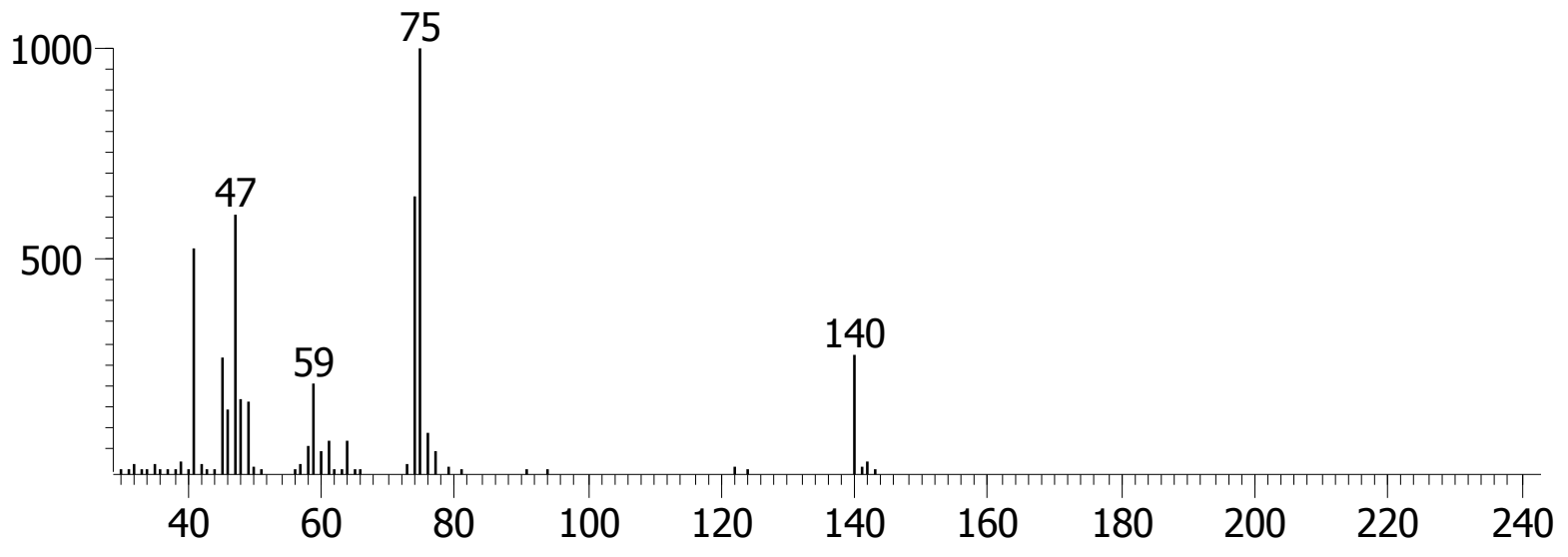

File $\quad:$ C:ILLNL2010\TOF74

Acquired: 14 Jan 2009 4:16:16 PM Sample: 0.5 ppm SBMTE

Peak True - sample "TOF:74", peak 116, at $774,2.660 \mathrm{sec}$, sec

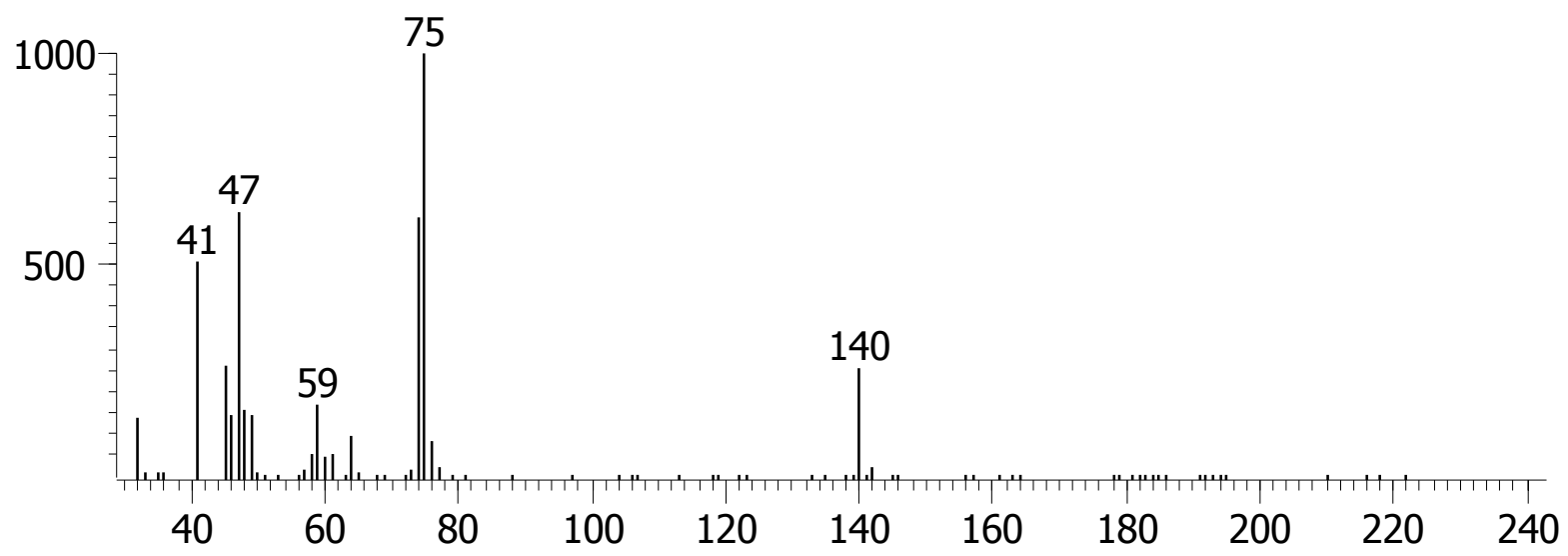

EI mass spectrum $(\mathrm{m} / \mathrm{z} 75,140)$ of:

Top: $\quad$ Spectrum of urine sample S5, retention time 771, $2.710 \mathrm{sec}$

Bottom: Reference standard of 1,1'-Sulfonylbis[2-9methylthio)ethane]. (MW: 232). 

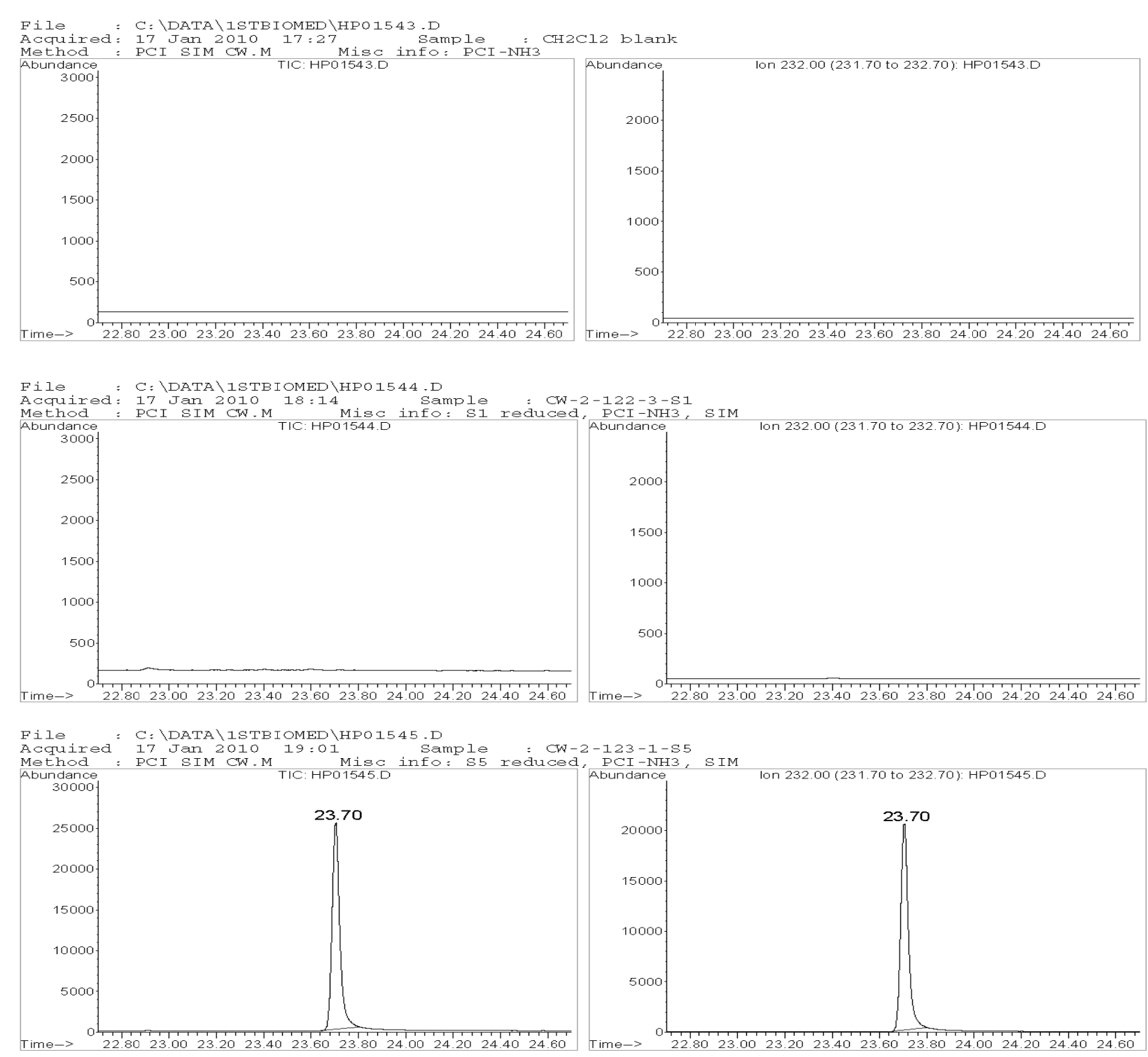

File : C: \DATA \ISTBIOMED $\backslash H P O 1546 . D$

Acquired: 17 Jar 2010 19:48
Method: PCI SIM CW.M Sample, SBMTE STD

Method : PCI SIM CW.M TIC:HP01546.D info: STD, PCI-NH3, SIM
Abundance
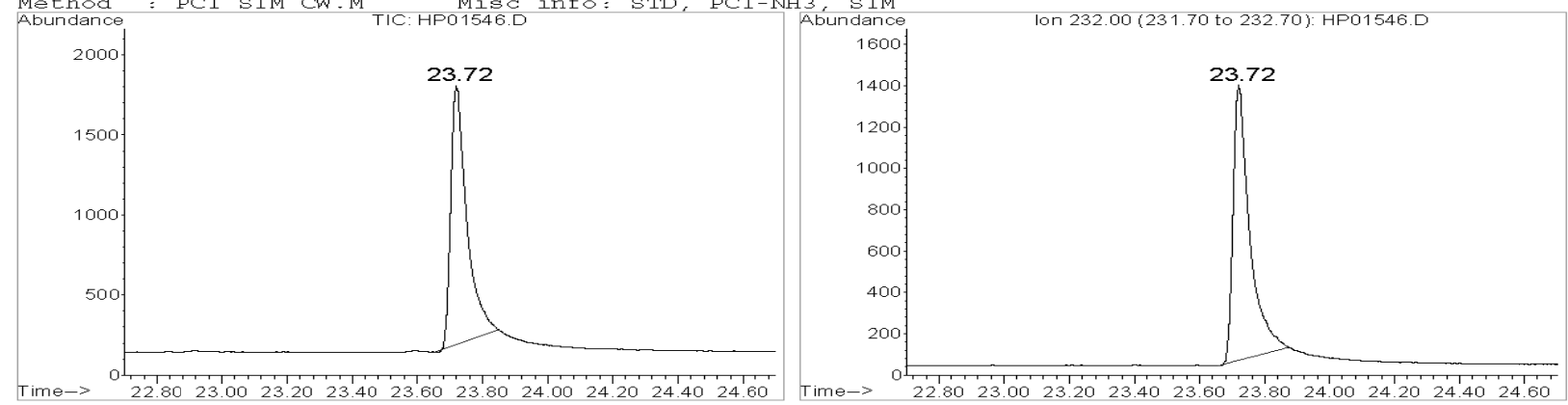

CI chromatograms supporting identification; TIC on left; EIC (m/z 232) on right.

Top: $\quad$ Chromatograms of solvent blank.

Second: Chromatograms of urine blank S1.

Third: $\quad$ Chromatograms of Urine sample S5, retention time $\mathbf{2 3 . 7 0}$ min.

Bottom: Chromatograms of reference standard of 1,1'-Sulfonylbis[2-(methylthio)ethane], retention time $23.72 \mathrm{~min}$. 


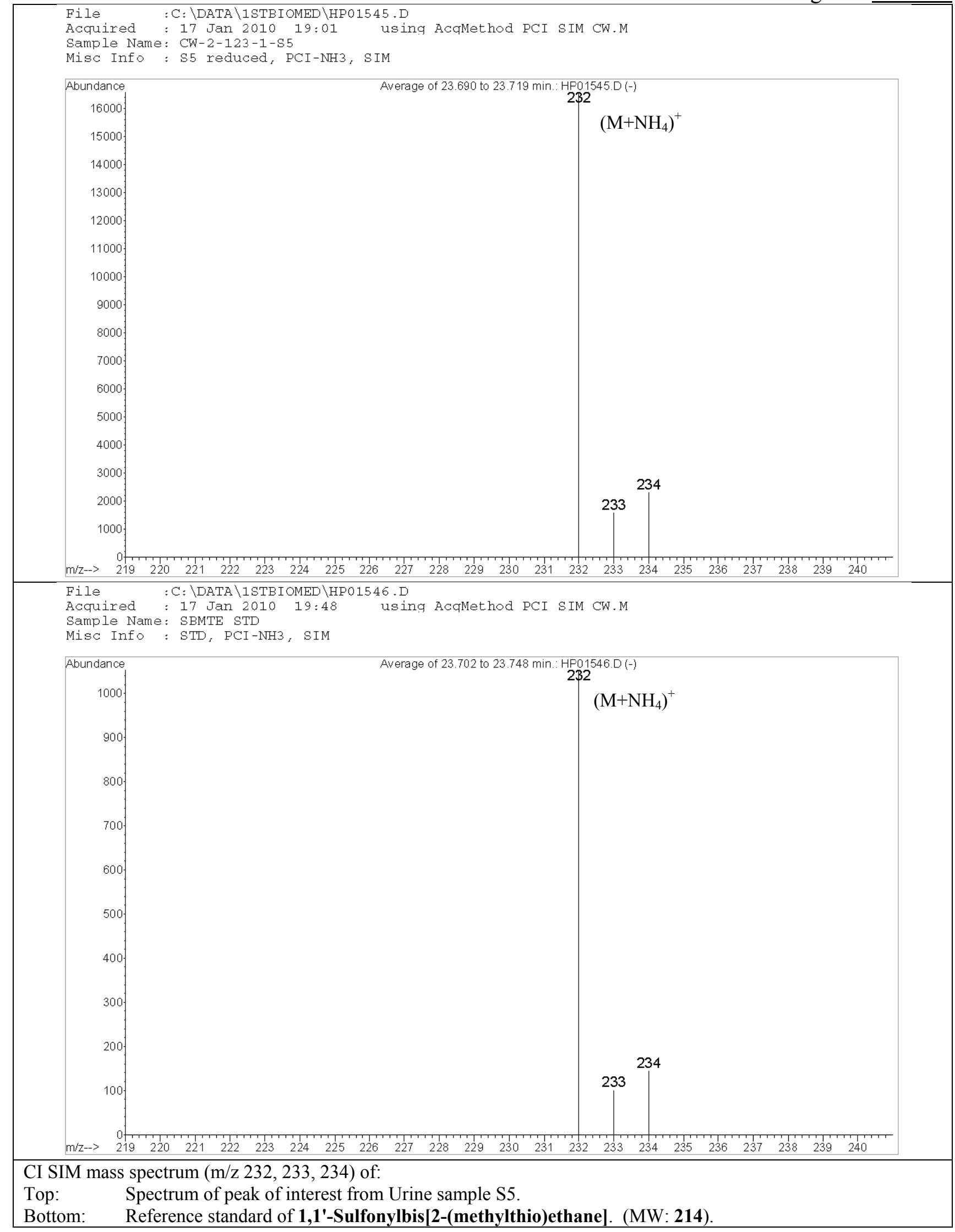


OPCW

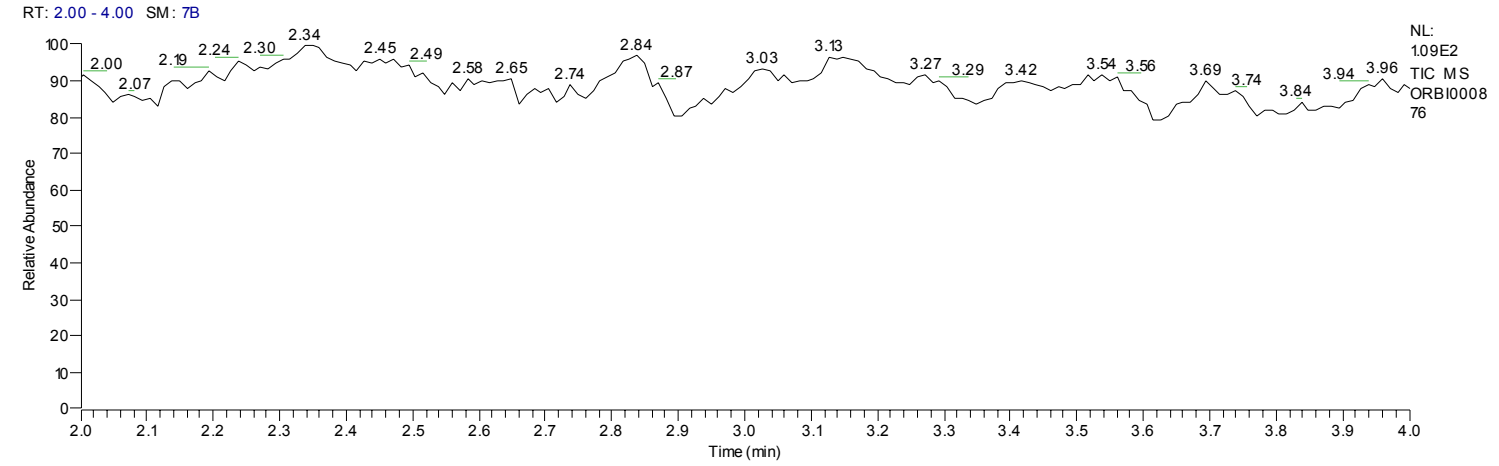

C:IXcaliburldatalORBI000877

1/17/2010 3:43:19 PM

CW-2-124-4-S

Pos, MSMS, Sun column, 200uL/min

RT: $2.00-4.00$ SM: $7 B$

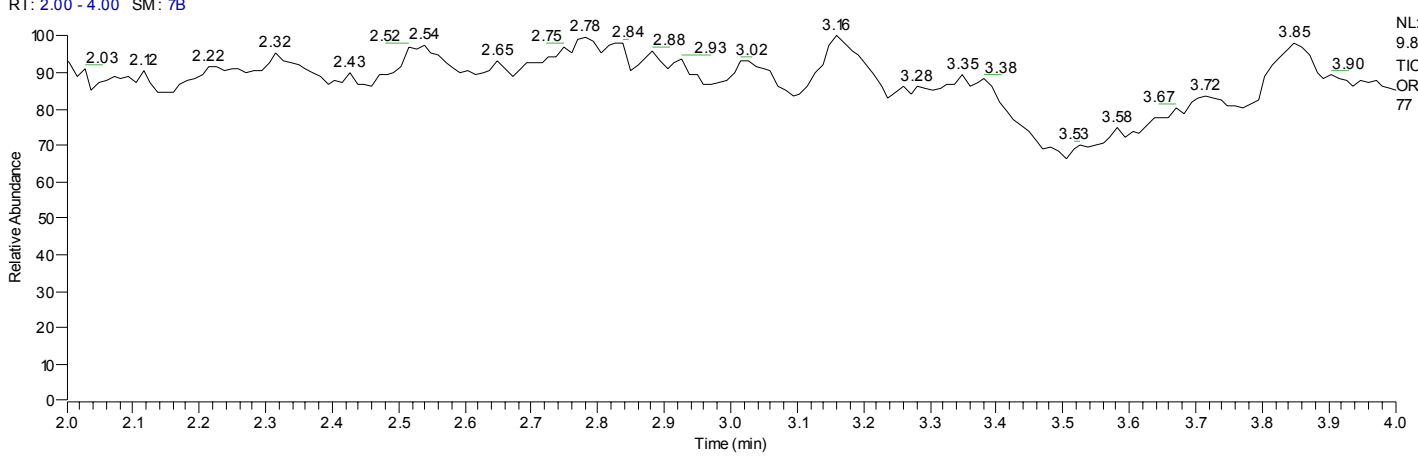

C:IXcaliburldatalORBI000878

1/17/2010 4:14:19 PM

CW-2-123-5-S5

Pos, MSMS, Sun column, 200uL/min

RT: $2.00-4.00 \mathrm{SM}: 7 \mathrm{~B}$

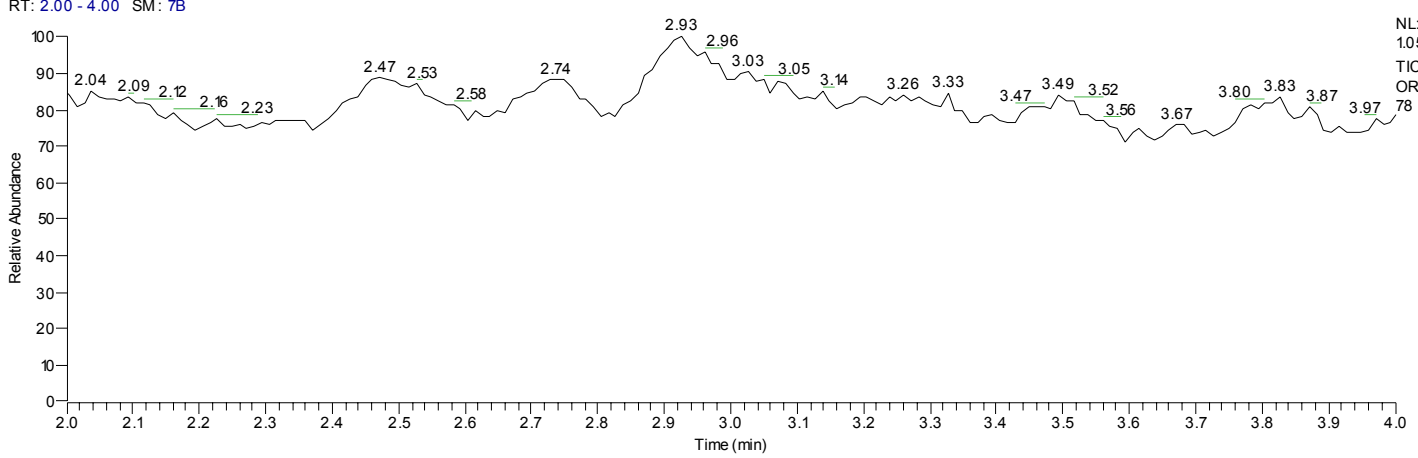

C:IXcaliburldatalORBI000879

1/17/2010 4:45:18 PM CW-2-114-3-STD (SBMSE)

Pos, MSMS, Sun column, 200uL/min

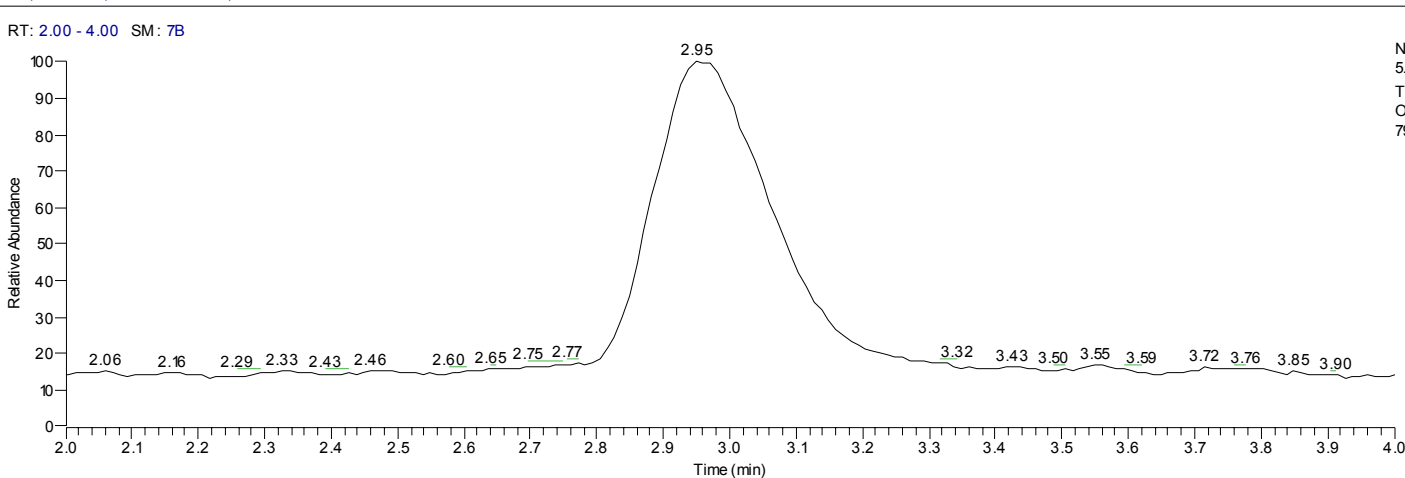

LC/MS/MS chromatograms. Top: water blank. Next: Urine blank S1. Third: Urine sample S5. Bottom: reference standard of 1,1'-Sulfonylbis[2-(methylsulfinyl)ethane]. 
OPCW

C:IXcaliburldatalORBI000876

Pos, MSMS, Sun column, 200uL/min

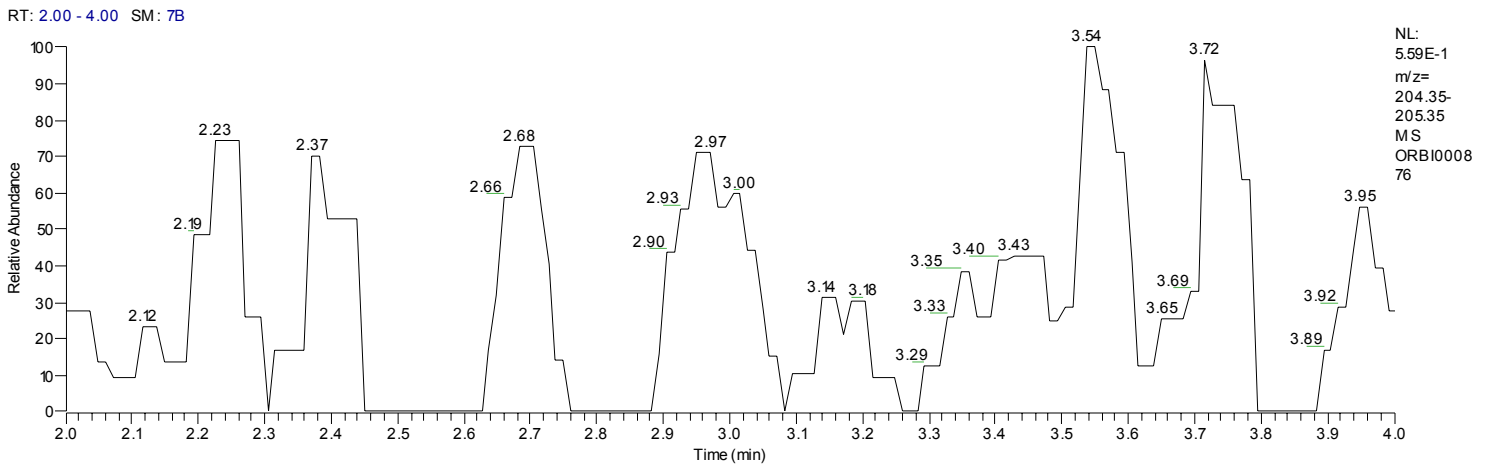

C:IXcaliburldatalORBI000877

Pos, MSMS, Sun column, 200uL/min

1/17/2010 3:43:19 PM

CW-2-124-4-S

RT: $2.00-4.00$ SM: $7 \mathrm{~B}$

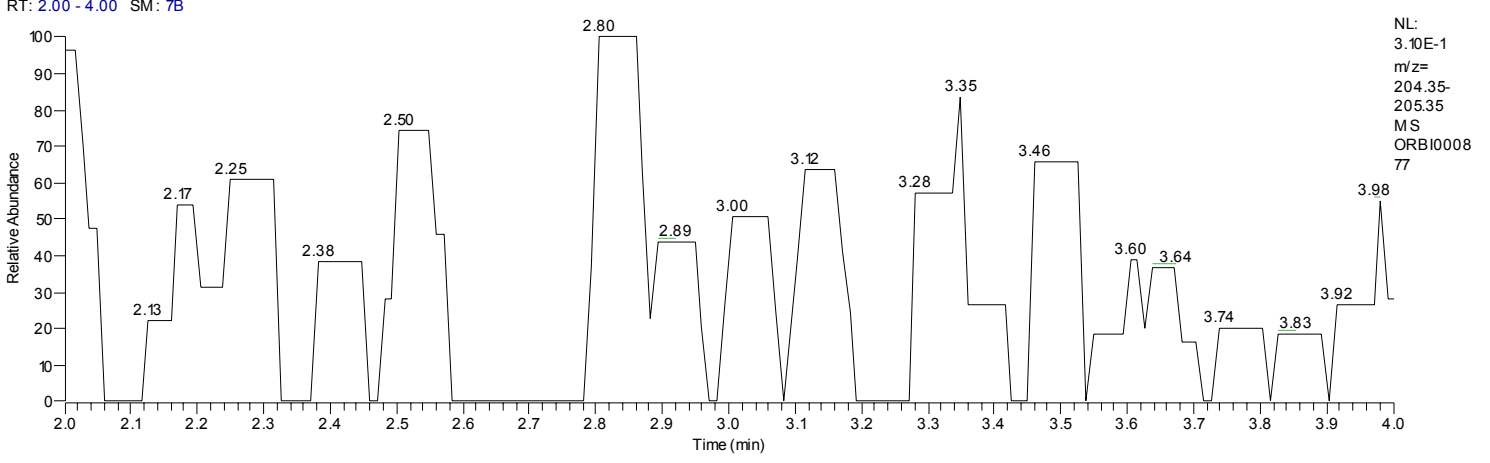

C:IXcaliburldatalORBI000878

Pos, MSMS, Sun column, 200uL/min

1/17/2010 4:14:19 PM

CW-2-123-5-S5

Page no.

32

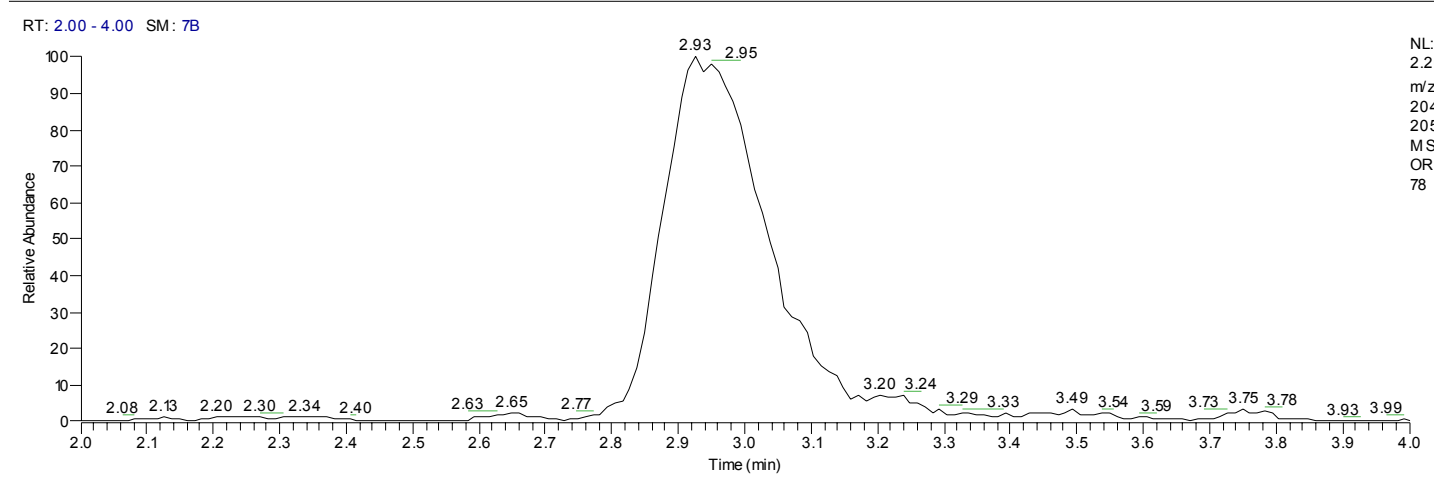

C:IXcaliburldatalORBI000879

1/17/2010 4:45:18 PM

CW-2-114-3-STD (SBMSE)

Pos, MSMS, Sun column, 200uL/min

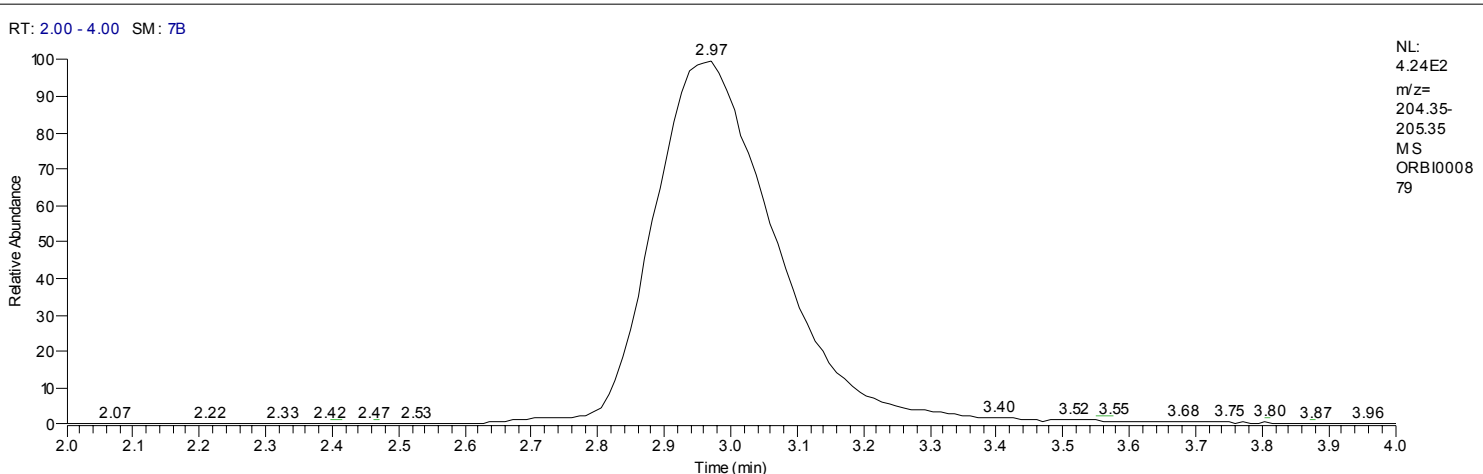

LC/MS/MS EIC (m/z 205) chromatograms. Top: water blank. Next: Urine blank S1. Third: Urine sample S5. Bottom: reference standard of 1,1'-Sulfonylbis[2-(methylsulfinyl)ethane]. 
ORBI000878 \#261-270 RT: 2.89-2.99 AV: 10 NL: $2.99 E 1$

T: ITMS + c ESI Full ms2 269.00@cid17.00 [70.00-280.00]

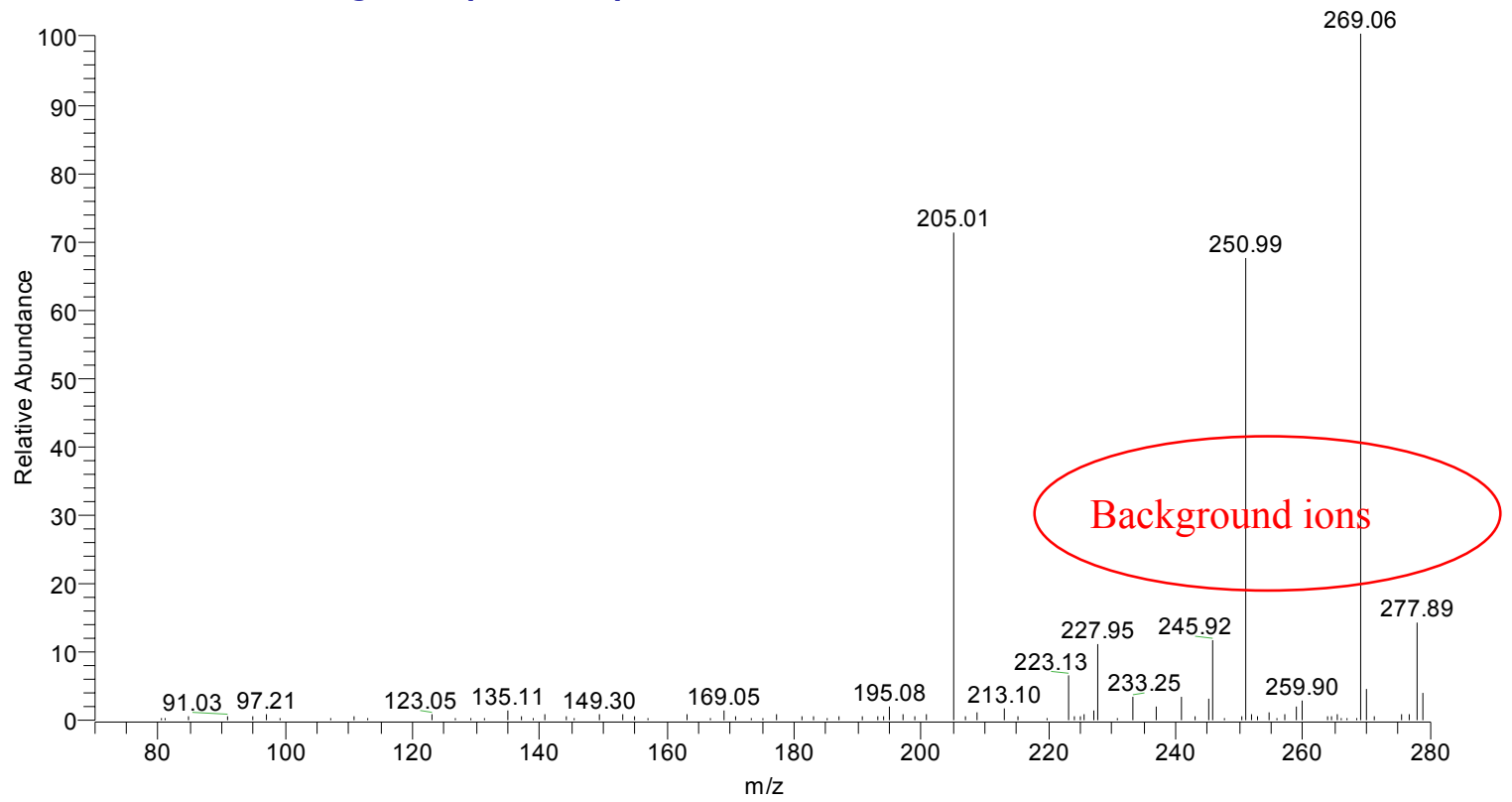

C:IXcaliburldatalORBI000879

1/17/2010 4:45:18 PM CW-2-114-3-STD (SBMSE)

Pos, MSMS, Sun column, 200uL/min

ORBI000879 \#261-274 RT: 2.89-3.04 AV: 14 NL: 3.76E2

T: ITMS + c ESI Full ms2 269.00@cid17.00 [70.00-280.00]

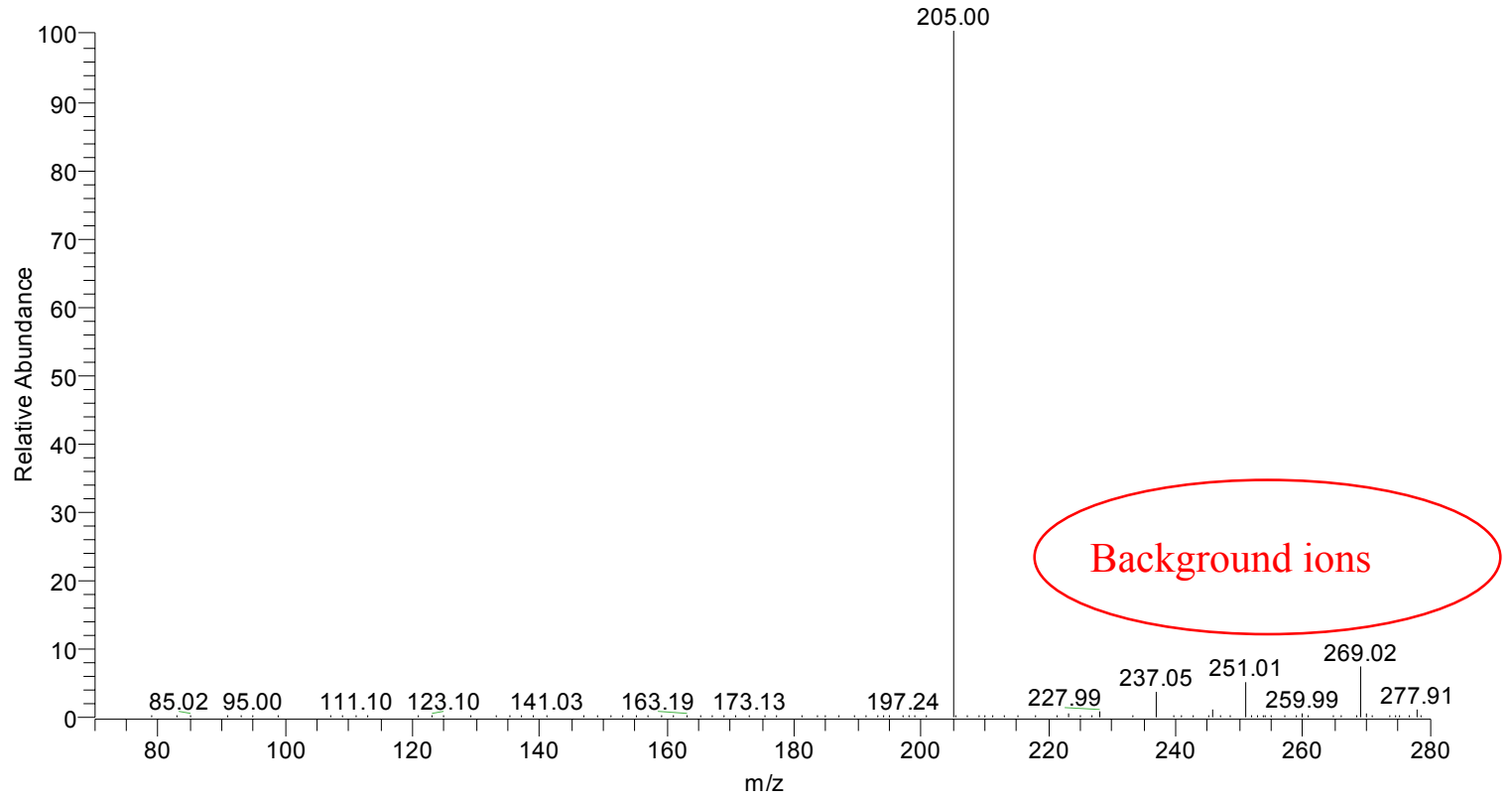

LC/MS/MS spectra - product spectra of parent $[\mathrm{M}+\mathrm{Na}]^{+} \mathrm{m} / \mathrm{z}$ 269. Top: Urine sample S5. Bottom: reference standard of 1,1'-Sulfonylbis[2-(methylsulfinyl)ethane]. 


\section{RESULTS: URINE SAMPLE S6}

$\beta$-Lyase metabolites of sulfur mustard were not found in this sample (after sample preparation that included $\mathrm{TiCl}_{3}$ reduction and analysis by GC-GC-MS (EI)).

Isopropyl methyphosophonate was found. The following few pages present the GC-MS (CI) data for the derivative and the LC-MS-MS data for the un-derivitized acid. 


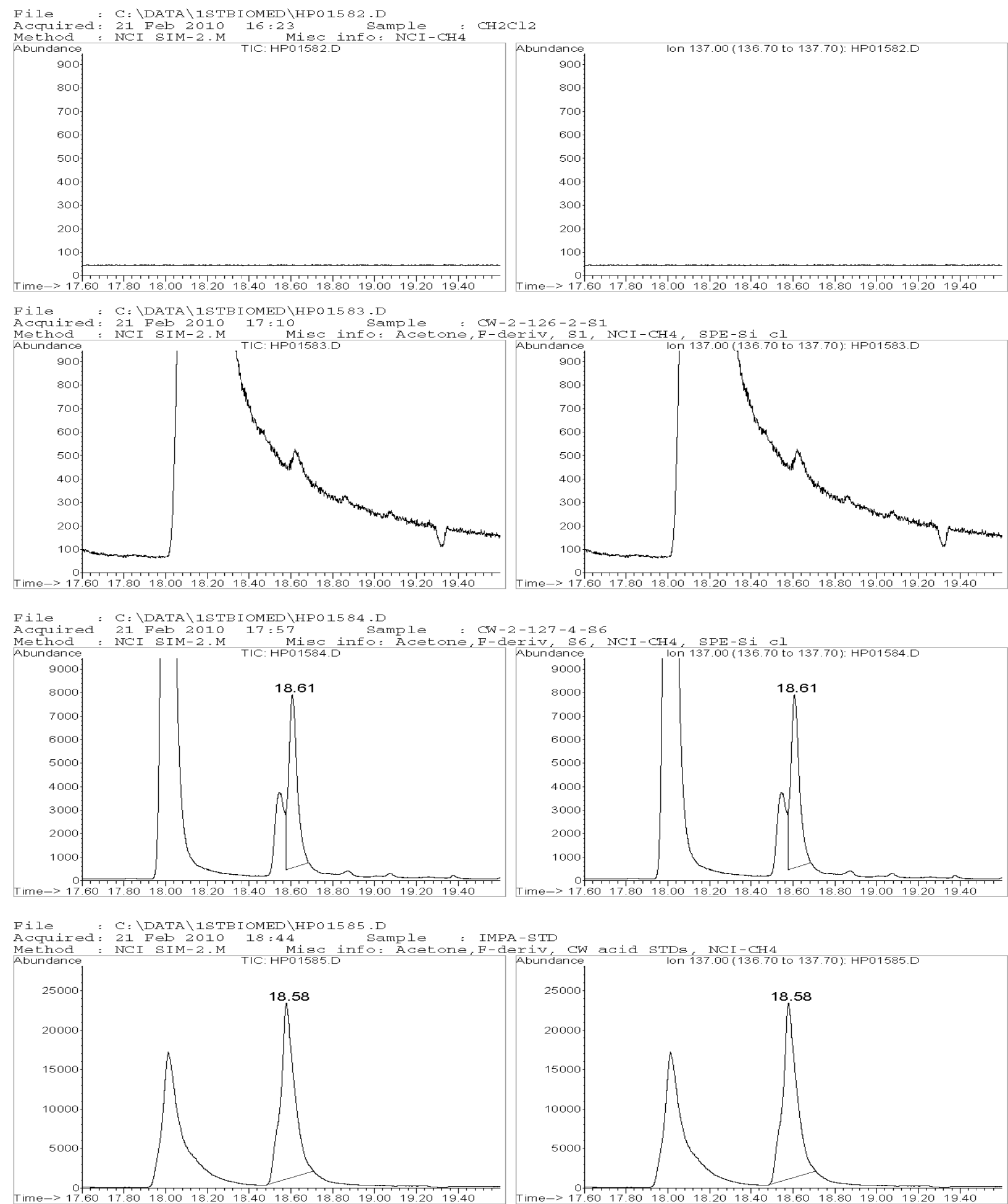

CI chromatograms supporting identification; TIC on left; EIC (m/z 137) on right.

Top: $\quad$ Chromatograms of solvent blank.

Second: Chromatograms of urine blank S1.

Third: $\quad$ Chromatograms of Urine sample S6, retention time $\mathbf{1 8 . 6 1}$ min.

Bottom: Chromatograms of reference standard of PFB derivative of Isopropyl methylphosphonate retention time $18.58 \mathrm{~min}$. 


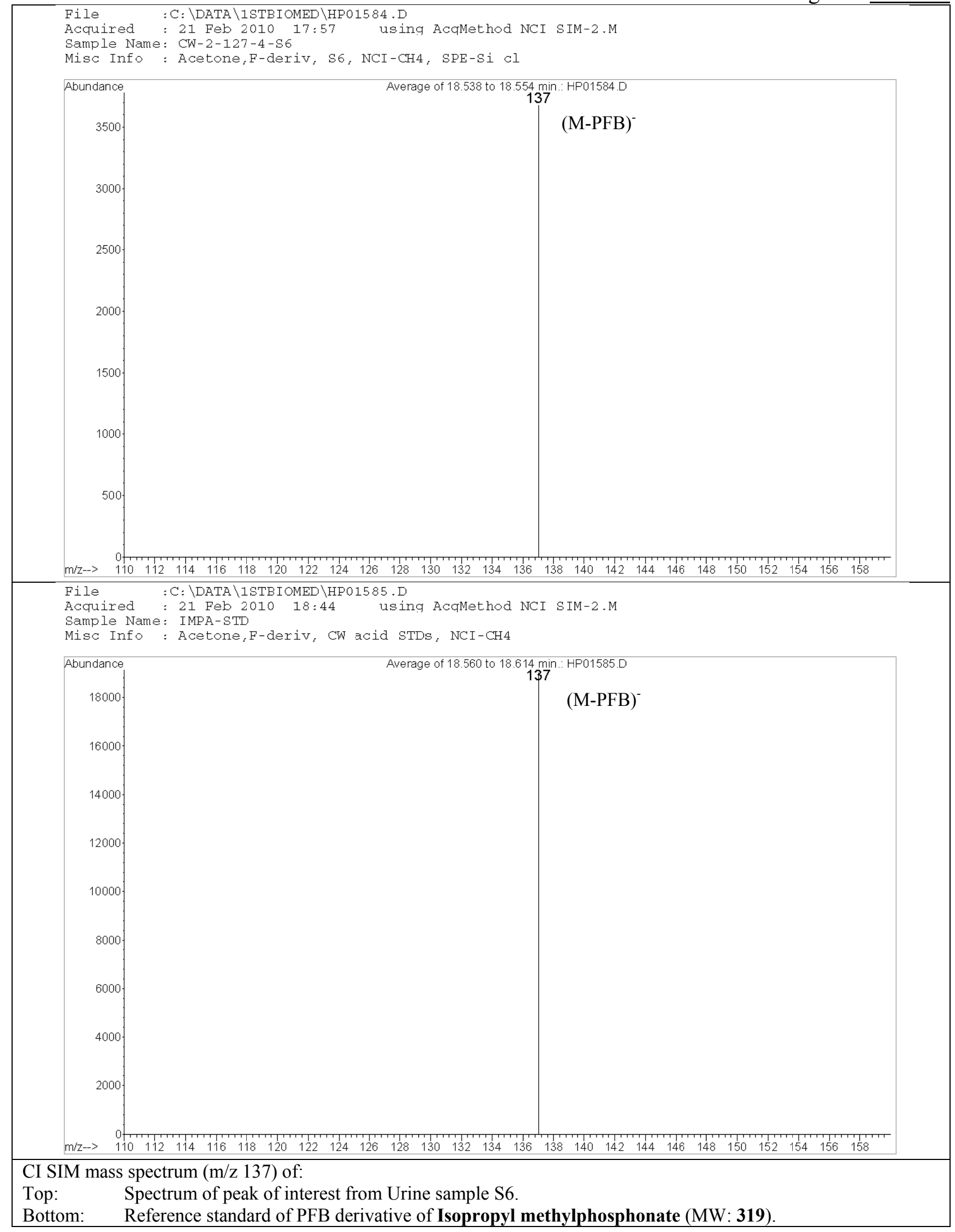




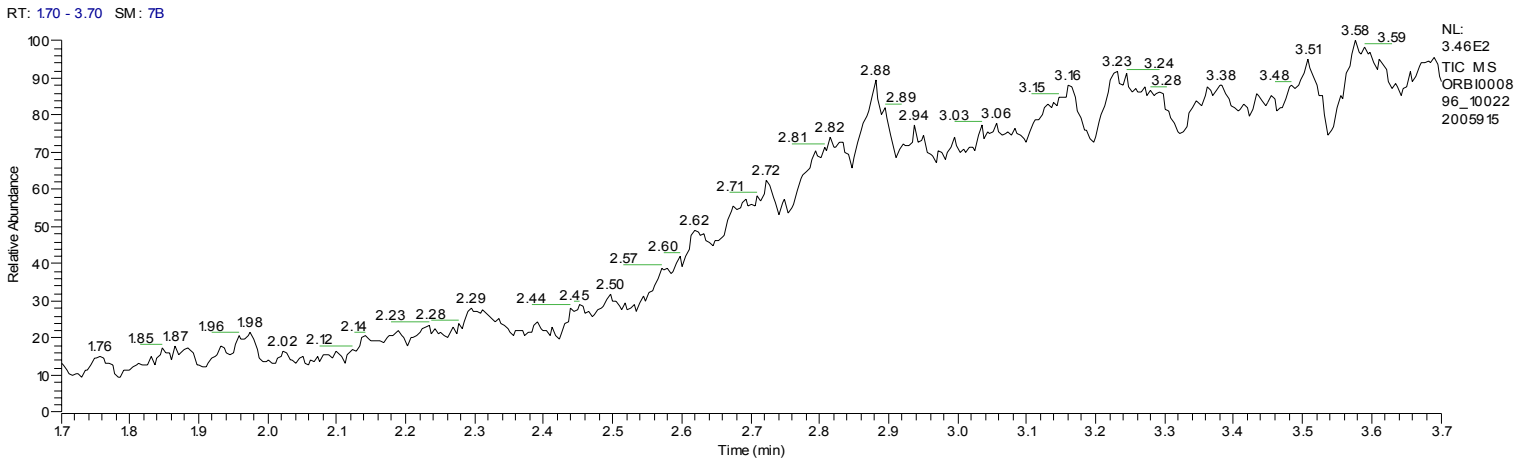

C:IXcaliburldatalORBI000897

2/22/2010 1:25:12 AM CW-2-127-6-S1

Neg, MSMS, T3 column,

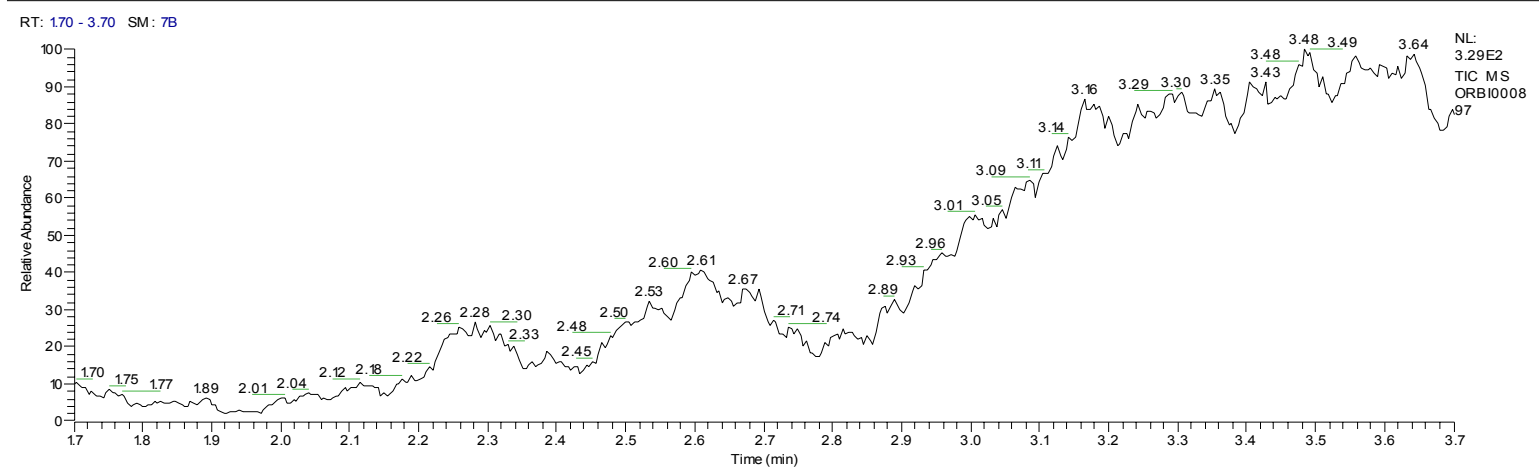

C:IXcaliburldatalORBI000898

2/22/2010 1:51:14 AM CW-2-128-3-S6

Neg, MSMS, T3 column, 200uL/min

RT: $1.70-3.70 \mathrm{SM}: 7 \mathrm{~B}$

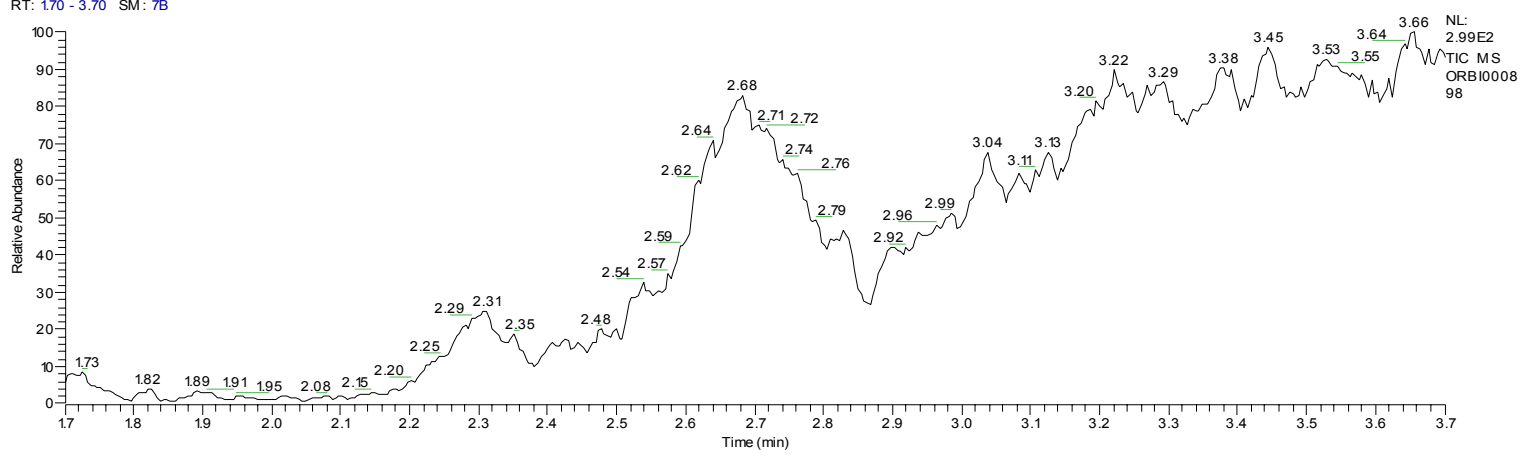

C:IXcaliburldatalORBI000899

2/22/2010 2:17:10 AM OW-2-128-5-STD, IMPA

Neg, MSMS, T3 column, 200uL/min

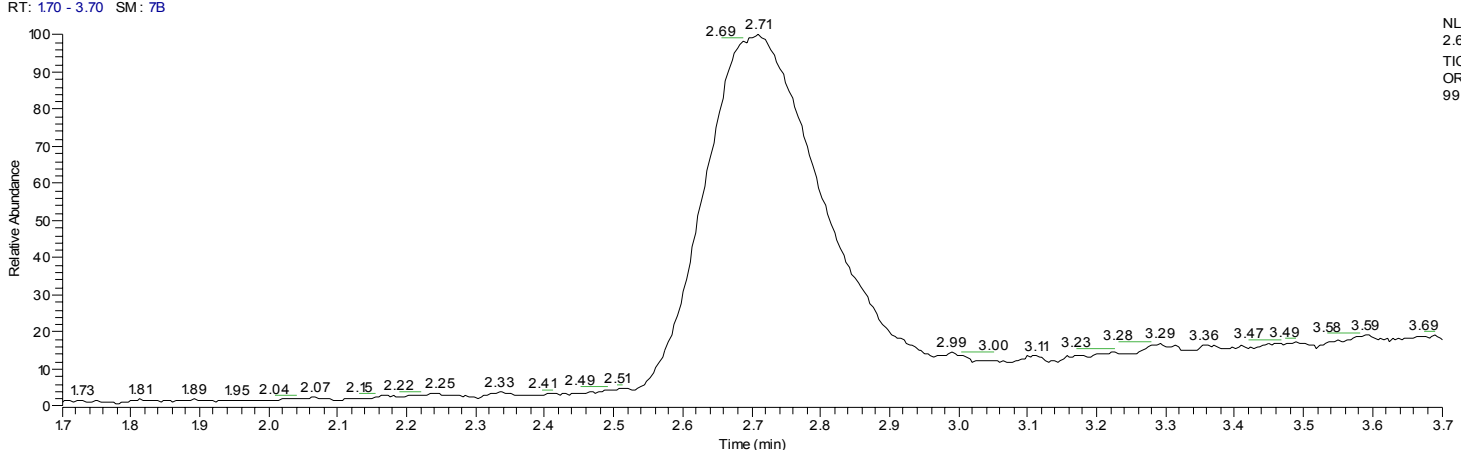

LC/MS/MS chromatograms. Top: water blank. Next: Urine blank S1. Third: Urine sample S6. Bottom: reference standard of Isopropyl methylphosphonate 


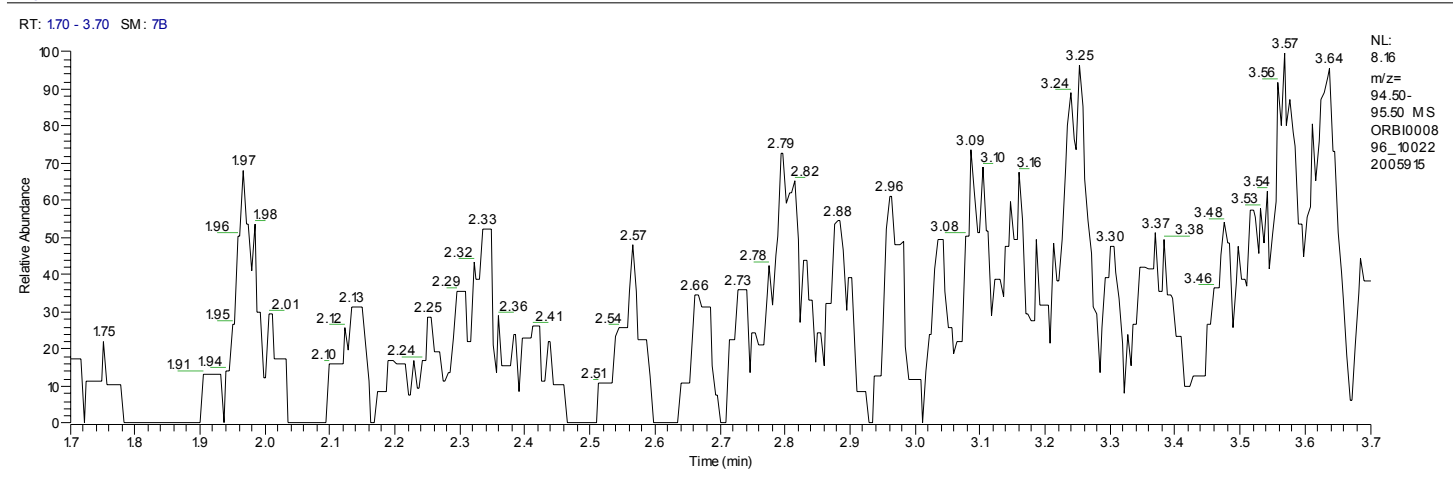

C:IXcaliburldatalORBI000897

2/22/2010 1:25:12 AM

CW-2-127-6-S1

Neg, MSMS, T3 column, 200uL/min

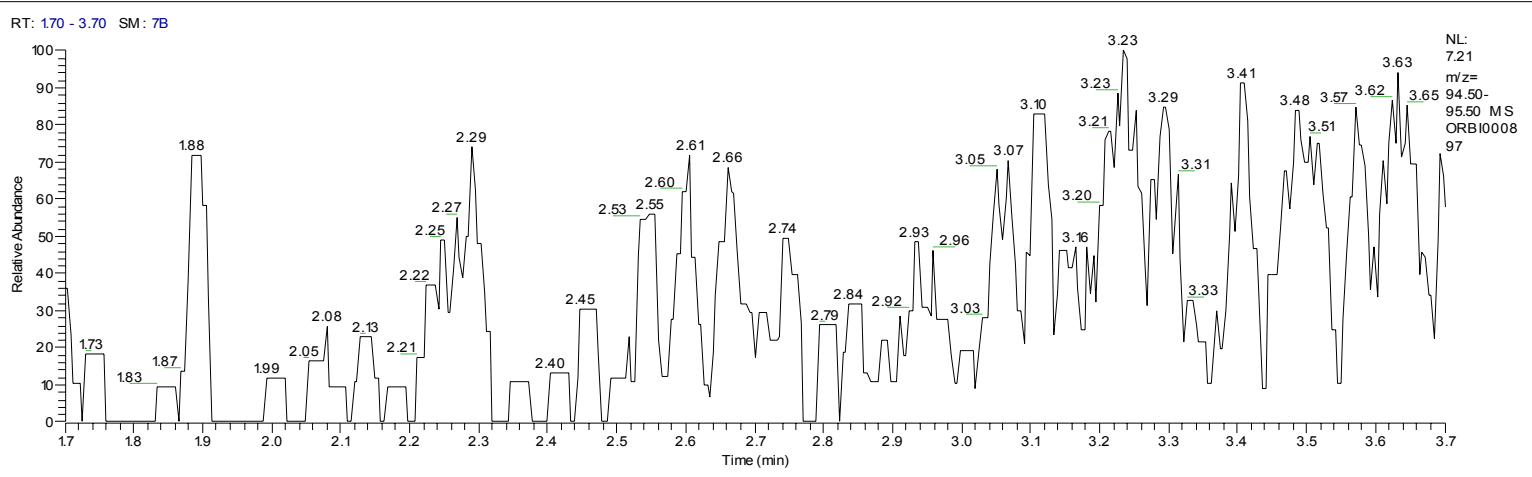

C:IXcaliburldatalORBI000898

2/22/2010 1:51:14 AM CW-2-128-3-S6

Neg, MSMS, T3 column, 200uL/min

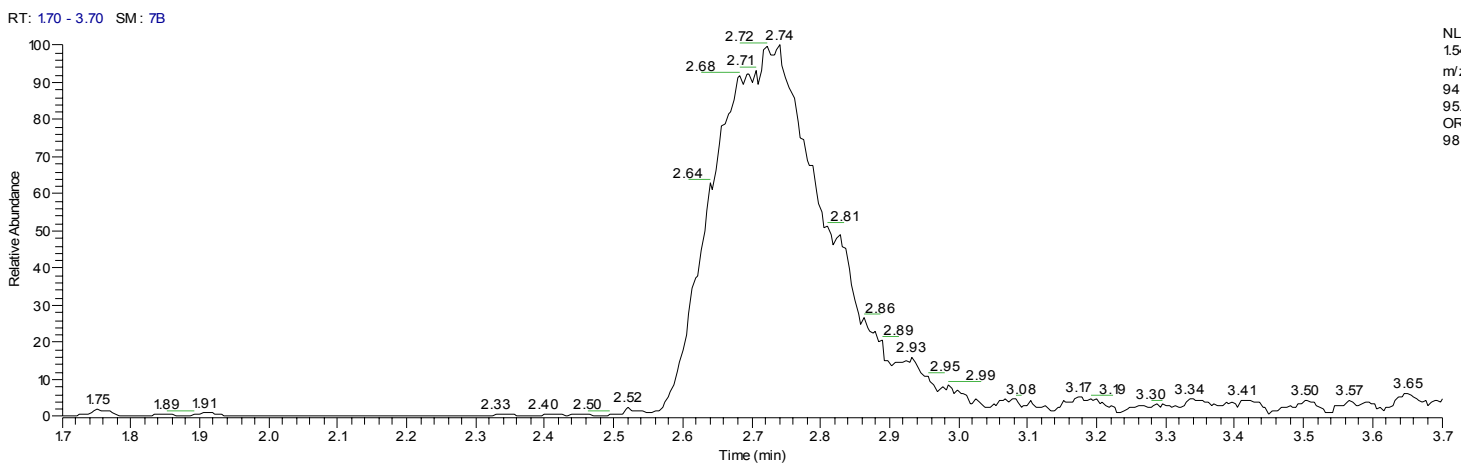

C:IXcaliburldatalORBI000899

2/22/2010 2:17:10 AM

Ww-2-128-5-STD, IMPA

Neg, MSMS, T3 column, 200uL/min

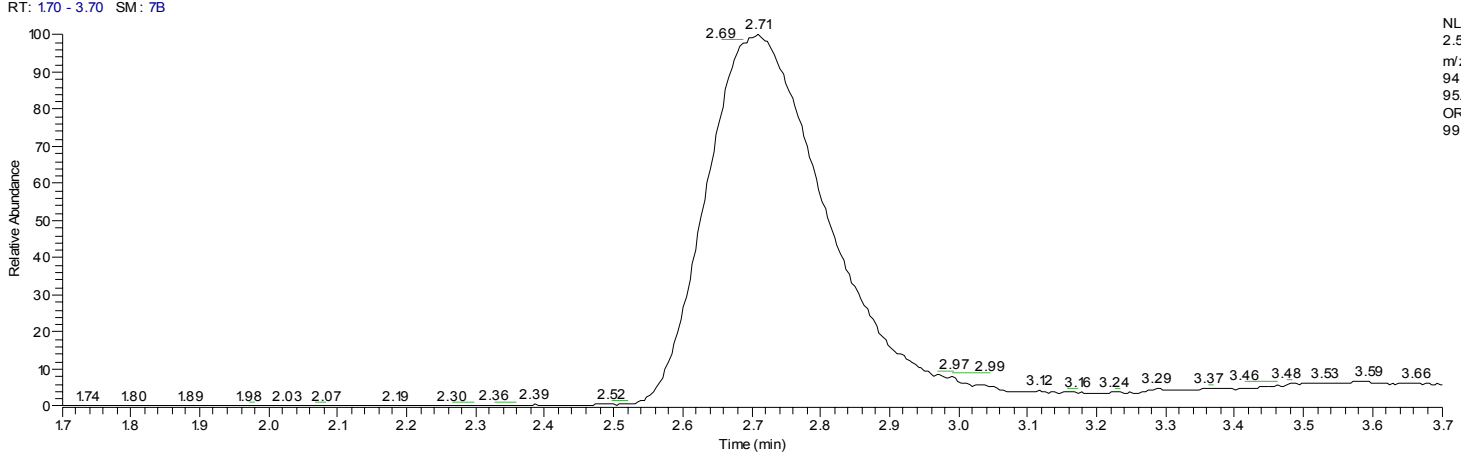

LC/MS/MS EIC (m/z 95) chromatograms. Top: water blank. Next: Urine blank S1. Third: Urine sample S6. Bottom: reference standard of Isopropyl methylphosphonate 
ORBI000898 \#603-626 RT: 2.64-2.74 AV: 24 NL: $1.32 E 2$

T: ITMS - c ESI Full ms2 137.00@cid33.00 [50.00-210.00]
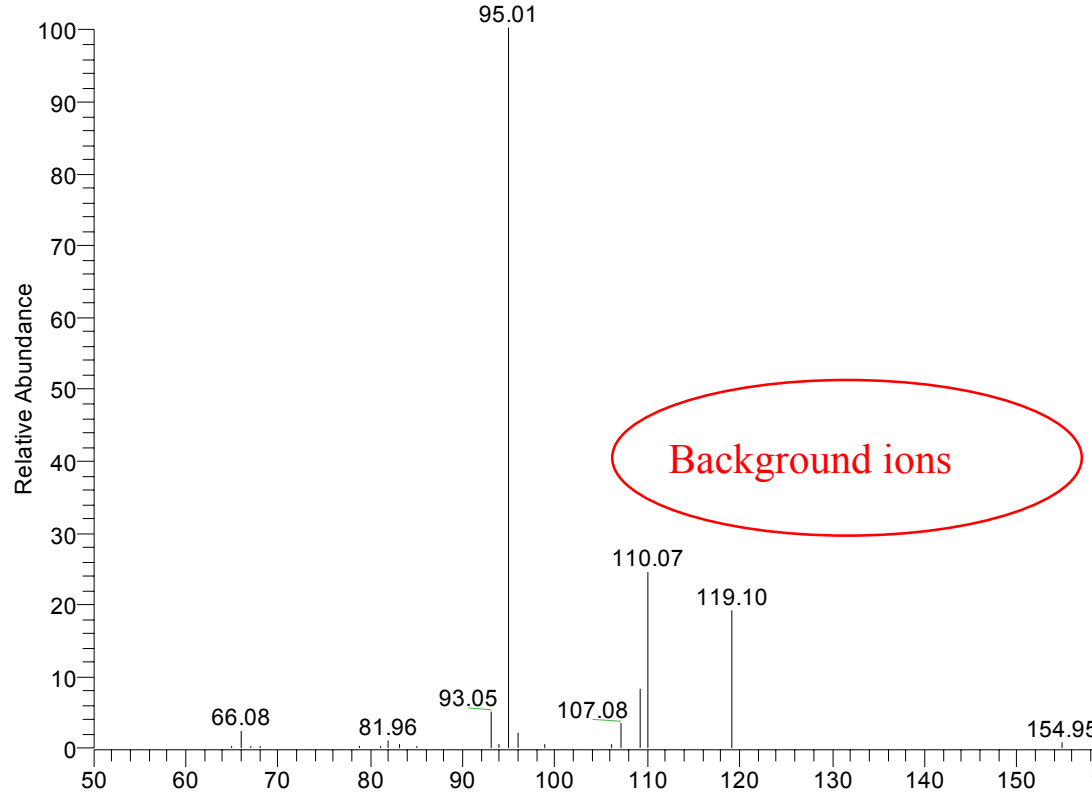

110.07

$\left.\right|^{110.07} 119.10$

119.10
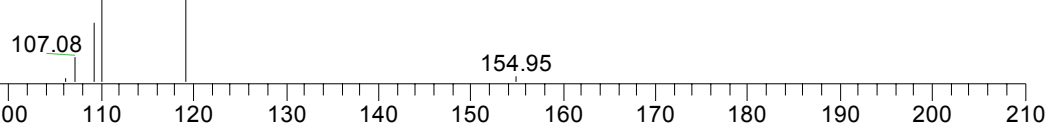

C:IXcaliburldatalORBI000899 Neg, MSMS, T3 column, 200uL/min

2/22/2010 2:17:10 AM CW-2-128-5-STD, IMPA

ORBI000899 \#604-637 RT: 2.64-2.78 AV: 34 NL: 2.22E3

T: ITMS - c ESI Full ms2137.00@cid33.00 [50.00-210.00]

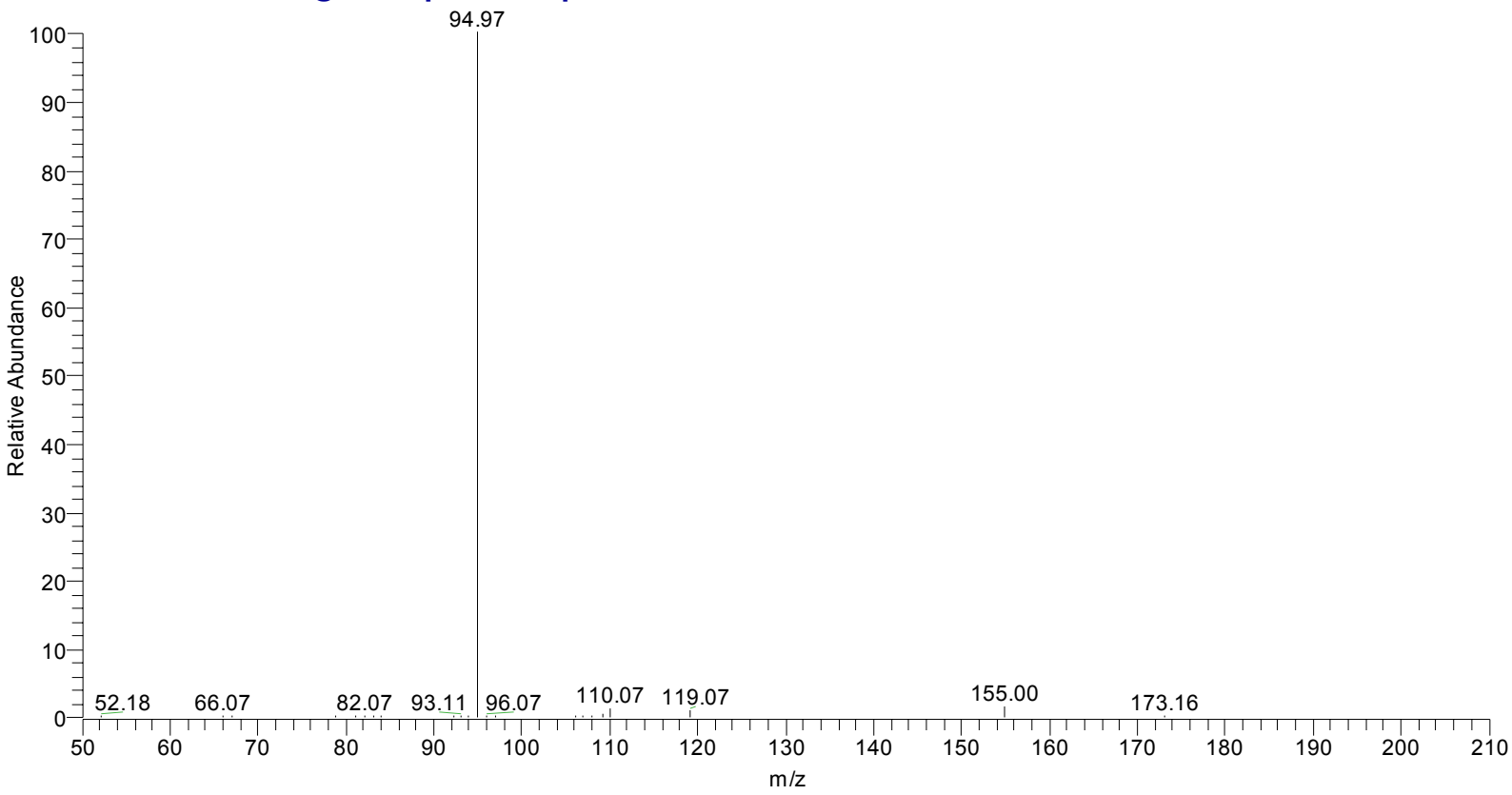

LC/MS/MS spectra - product spectra of parent $[\mathrm{M}-\mathrm{H}]^{-} \mathrm{m} / \mathrm{z}$ 137. Top: Urine sample S6. Bottom: reference standard of Isopropyl methylphosphonate 


\section{RESULTS: URINE SAMPLE S7}

A $\beta$-Lyase metabolite of sulfur mustard was found in this sample (after sample preparation that included $\mathrm{TiCl}_{3}$ reduction and analysis by GC-GC-MS (EI)) and confirmed with GC-MS (CI) and LC-MS-MS.

Present was 1,1'-Sulfonylbis[2-(methylsulfinyl)ethane] (SBMSE), but no evidence of 1Methylsulfinyl-2-[2-(methylthio)ethylsulfonyl]ethane (MSMTESE) was observed.

None of the phosphonic acids were found.

The following few pages present the GC-GC-MS (EI) and GC-MS (CI) data for the derivative and the LC-MS-MS data for the un-derivitized metabolite. 
File : C:LLNL2010\TOF75

Acquired: 14 Jan 2009 4:52:51 PM Sample: CH2Cl2 Blank Lot\# 7284M

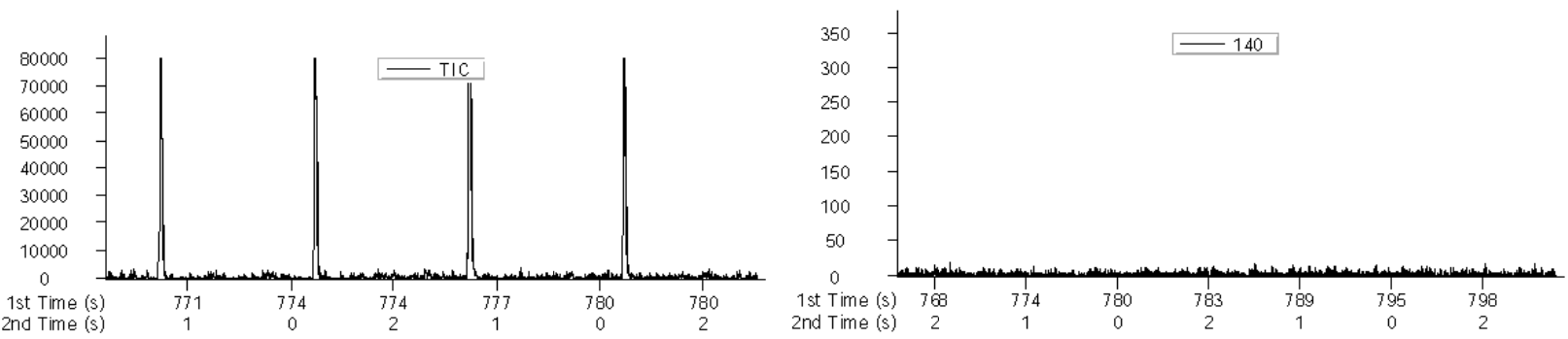

File : C:LLNL2010 \TOF76

Acquired: 14 Jan 2009 5:29:26 PM Sample: CW-2-122-3-S1

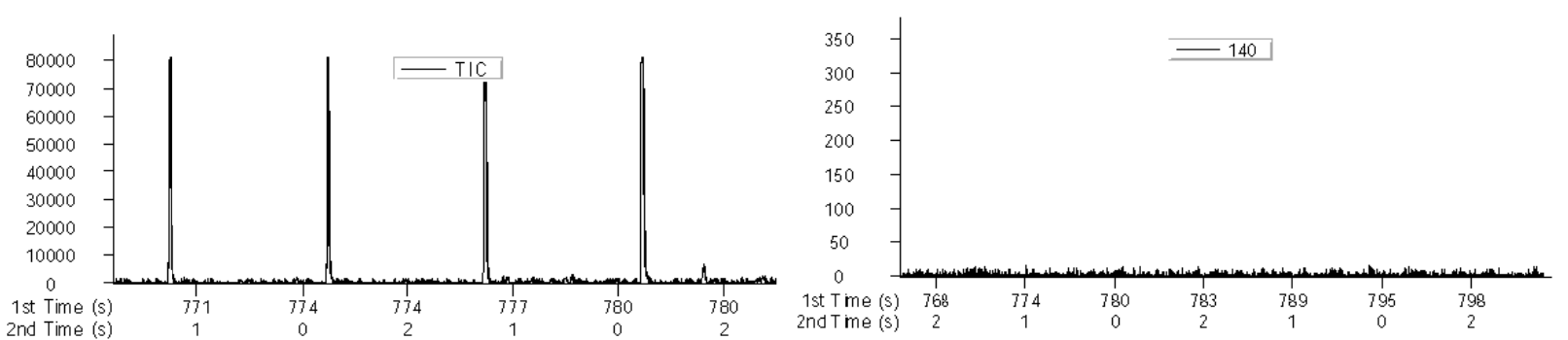

File : C:LLNL2010 \TOF77

Acquired: 14 Jan 2009 6:05:59 PM Sample: CW-2-123-3-S7

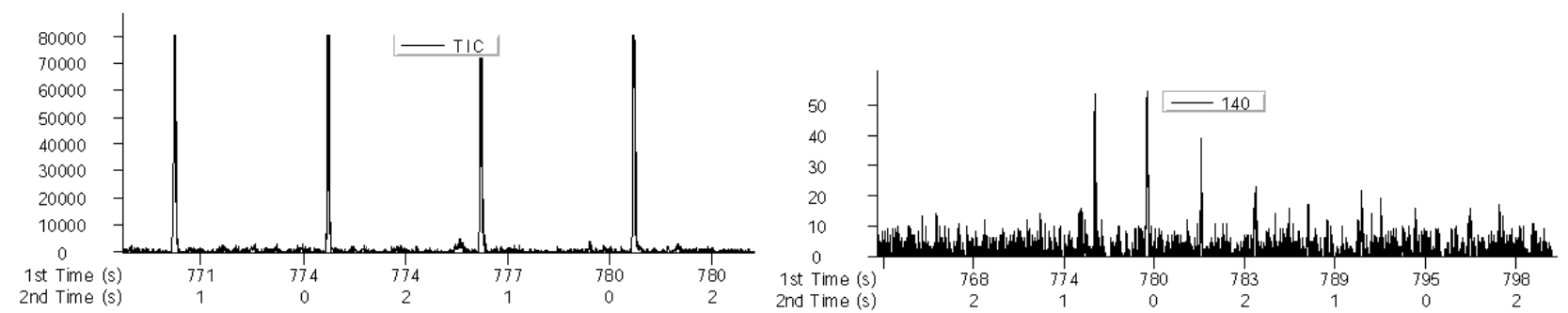

File $\quad$ : C:LLNL2010\TOF78

Acquired: 14 Jan 2009 4:16:16 PM Sample: 0.5 ppm SBMTE

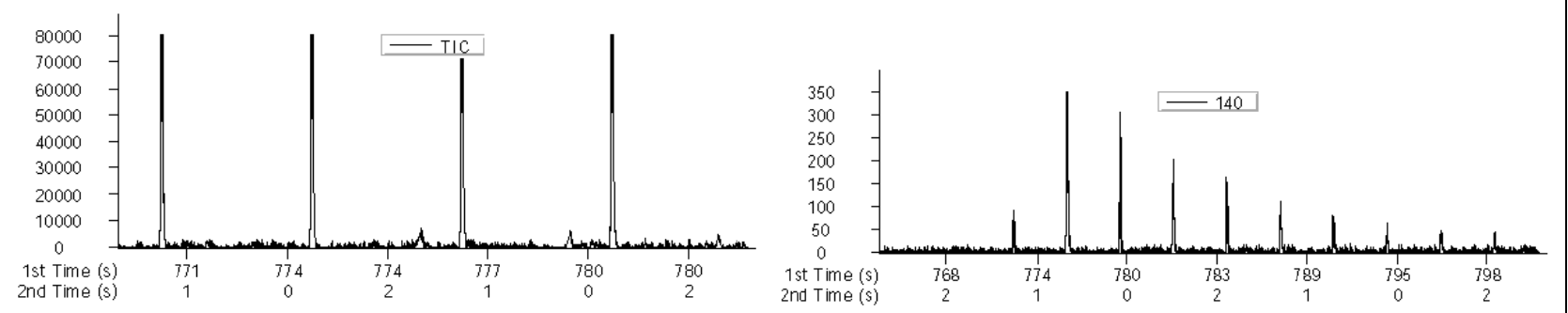

EI chromatograms supporting identification; TIC on left; EIC (m/z 140) on right

Top: Chromatograms of solvent blank.

Second: Chromatograms of urine blank S1.

Third: Chromatograms or urine sample S7, retention time 774, $2.658 \mathrm{sec}$

Bottom: Chromatograms of reference standard of 1,1'-Sulfonylbis[2-9methylthio)ethane], retention time 774, 2.655 sec 


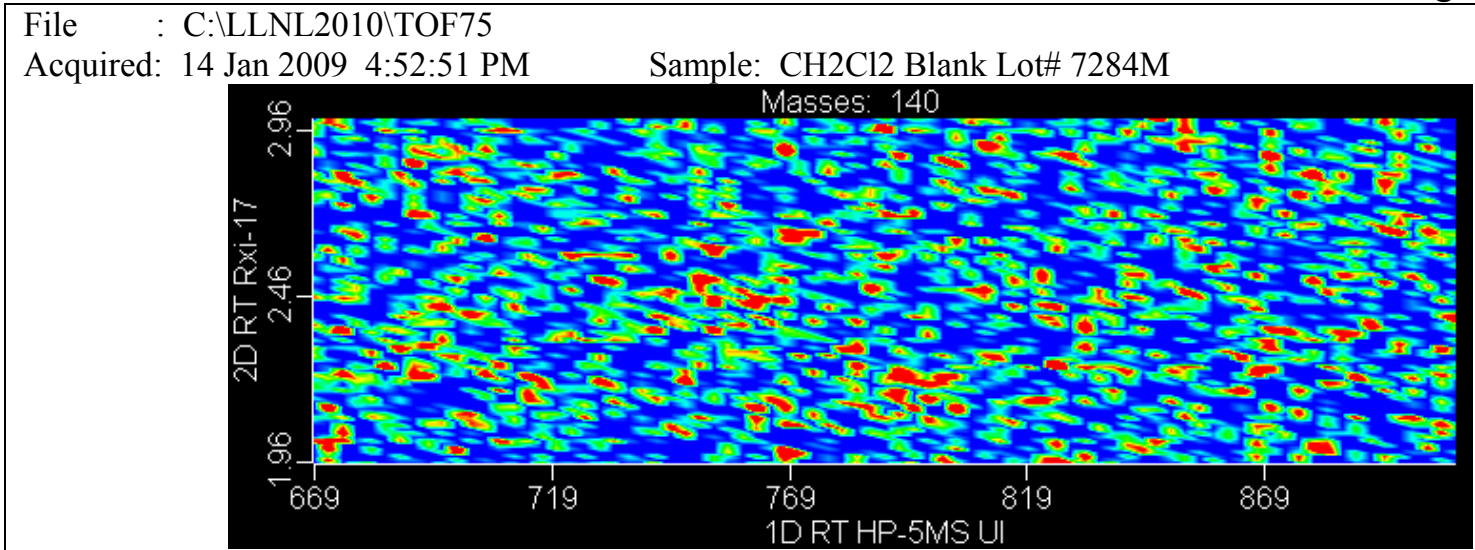

File : C:ILLNL2010\TOF76

Acquired: 14 Jan 2009 5:29:26 PM Sample: CW-2-122-3-S1

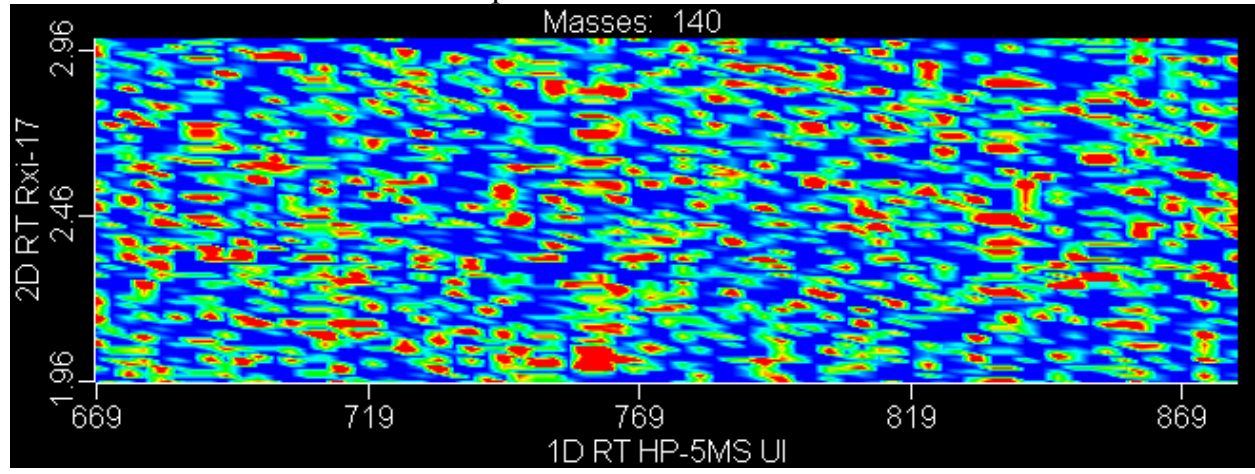

File $\quad$ : C:ILLNL2010\TOF77

Acquired: 14 Jan 2009 6:05:59 PM Sample: CW-2-123-3-S7

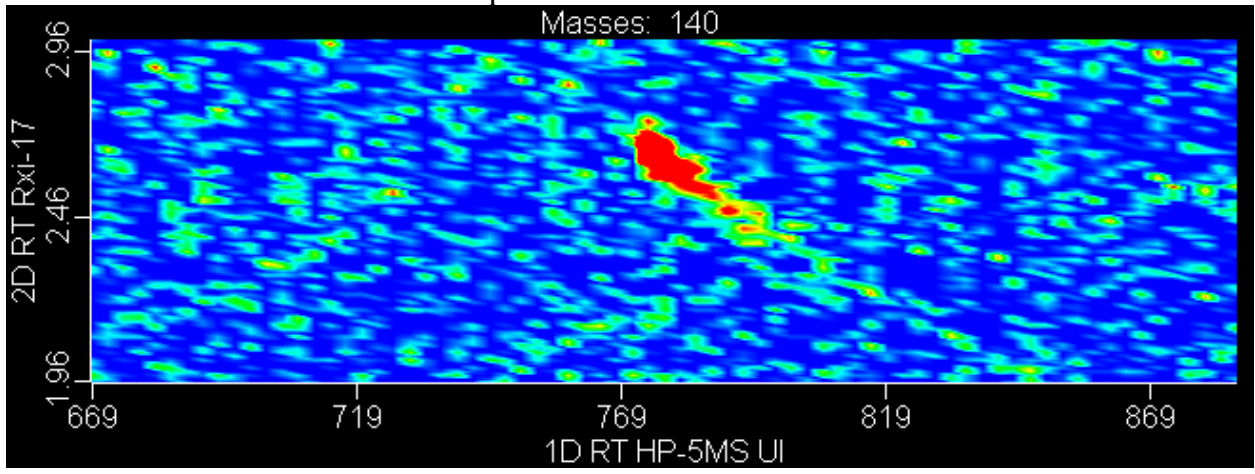

File $\quad$ : C:ILLNL2010\TOF78

Acquired: 14 Jan 2009 4:16:16 PM Sample: 0.5 ppm SBMTE

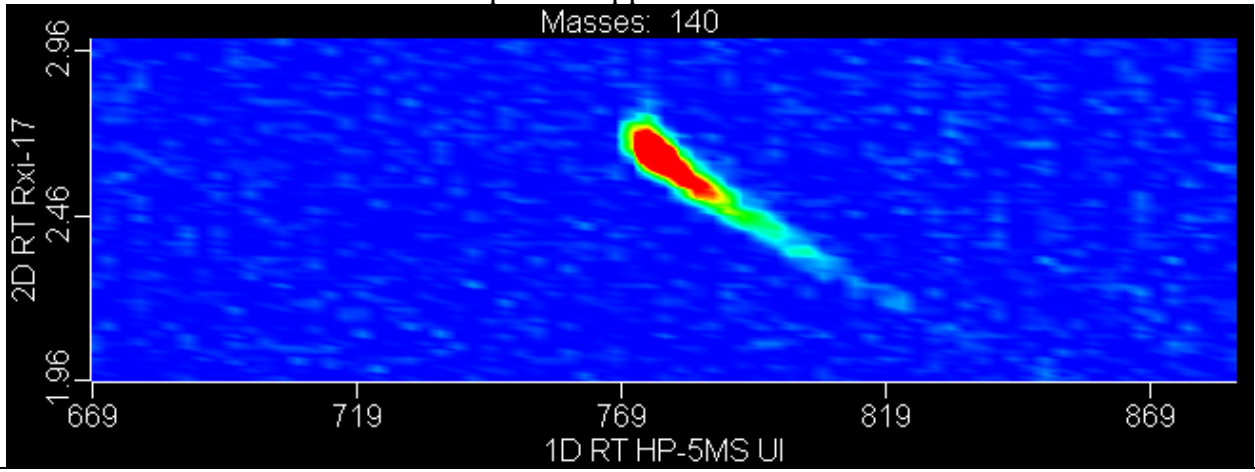

2D Contour plot supporting identification; EIC (m/z 140)

Top: Contour plot of solvent blank; Second: Contour plot of urine blank S1; Third: Contour plot of urine sample S7, retention time 774, $2.658 \mathrm{sec}$; Bottom: Contour plot of reference standard of 1,1'-Sulfonylbis[2-9methylthio)ethane], retention time $774,2.655 \mathrm{sec}$ 
File : C: \LLNL2010\TOF77

Acquired: 14 Jan 2009 6:05:59 PM

Sample: CW-2-123-3-S7

Peak True - sample "TOF:77", peak 150, at 774 , $2.658 \mathrm{sec}$, sec

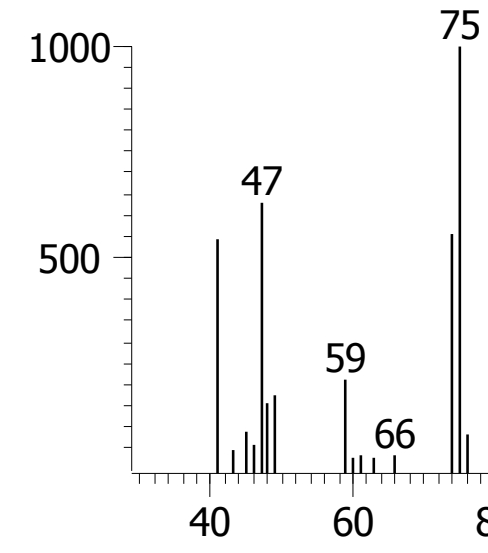

75

107

$\begin{array}{llll}80 & 100 & 120 & 140\end{array}$
File : C: $:$ LLNL2010\TOF78

Acquired: 14 Jan 2009 4:16:16 PM

Peak True - sample "TOF:78", peak 120, at 774 , 2.655 sec , sec

Sample: 0.5 ppm SBMTE $\begin{array}{lllll}160 & 180 & 200 & 220 & 240\end{array}$

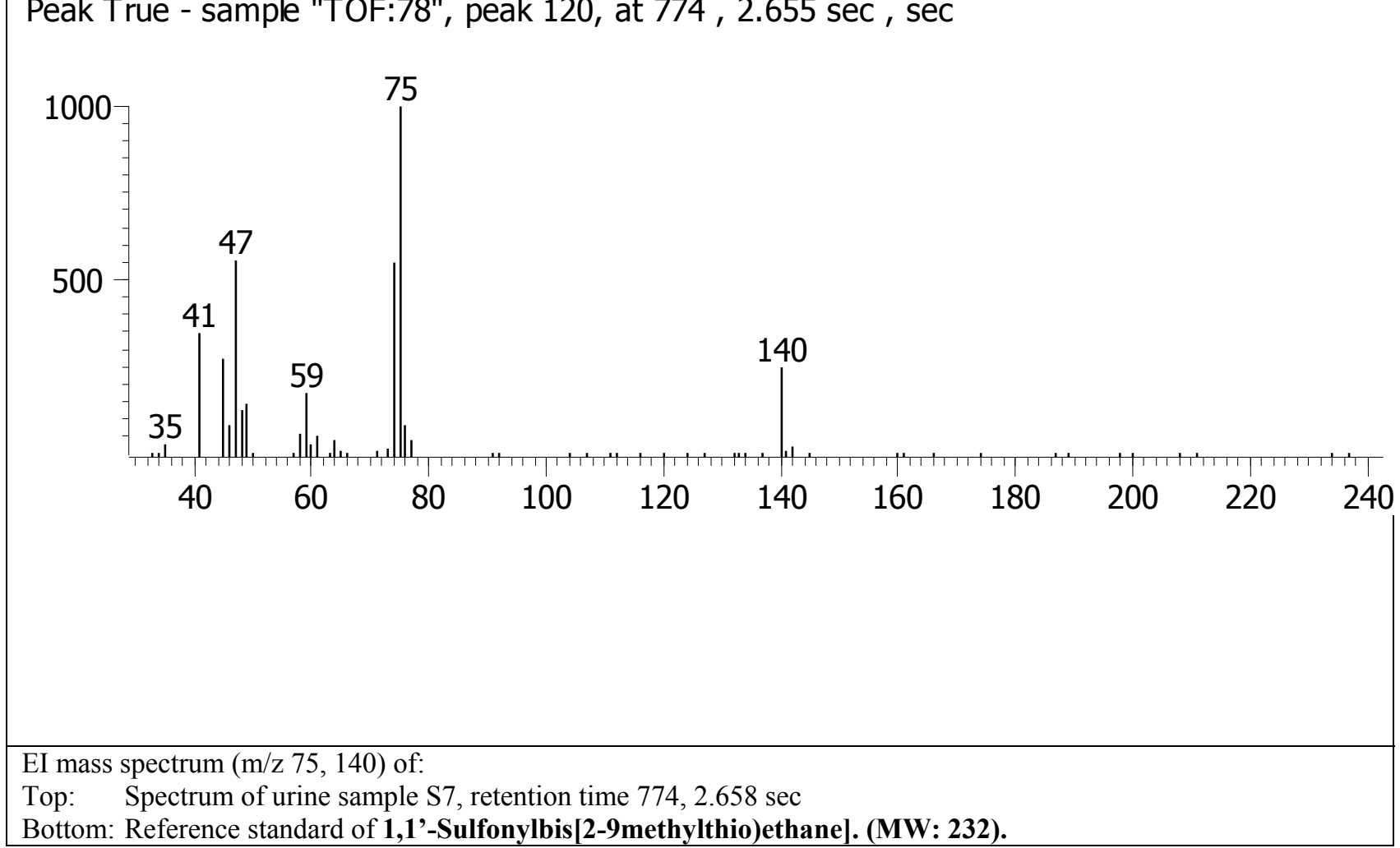



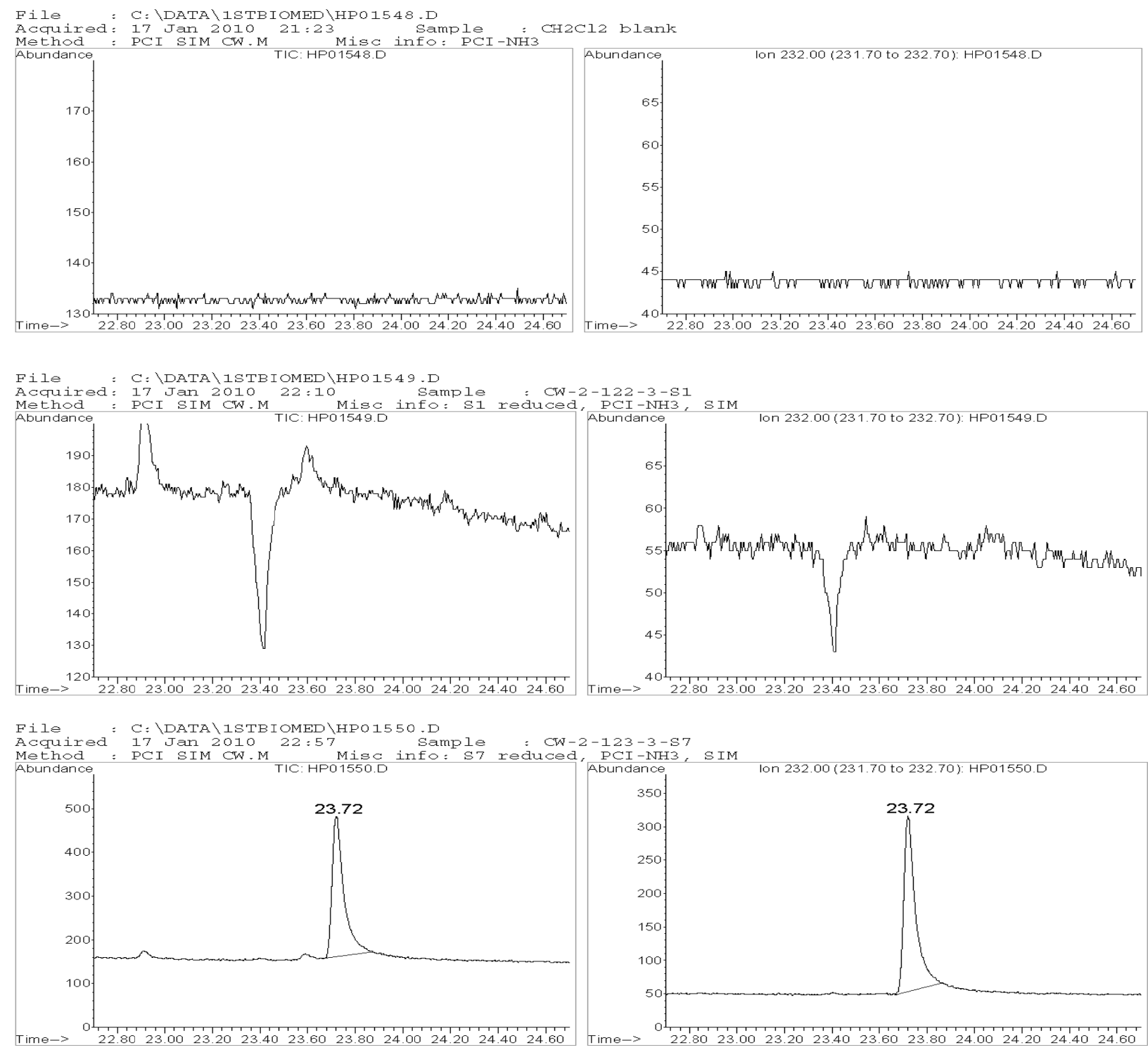

File : C: \DATA \STBIOMED \HPOL551.D

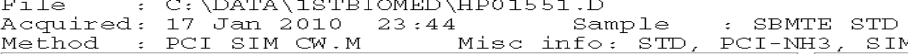

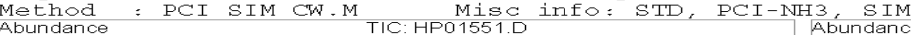

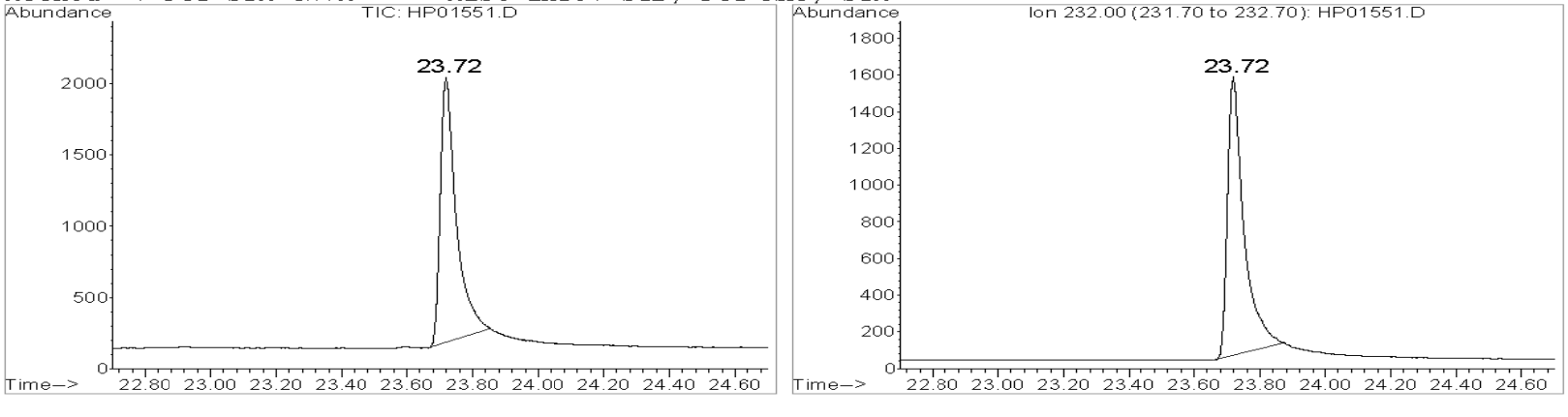

CI chromatograms supporting identification; TIC on left; EIC $(\mathrm{m} / \mathrm{z}$ 232) on right.

Top: $\quad$ Chromatograms of solvent blank.

Second: Chromatograms of urine blank S1.

Third: $\quad$ Chromatograms of Urine sample S7, retention time $\mathbf{2 3 . 7 2}$ min.

Bottom: Chromatograms of reference standard of 1,1'-Sulfonylbis[2-(methylthio)ethane], retention time $23.72 \mathrm{~min}$. 


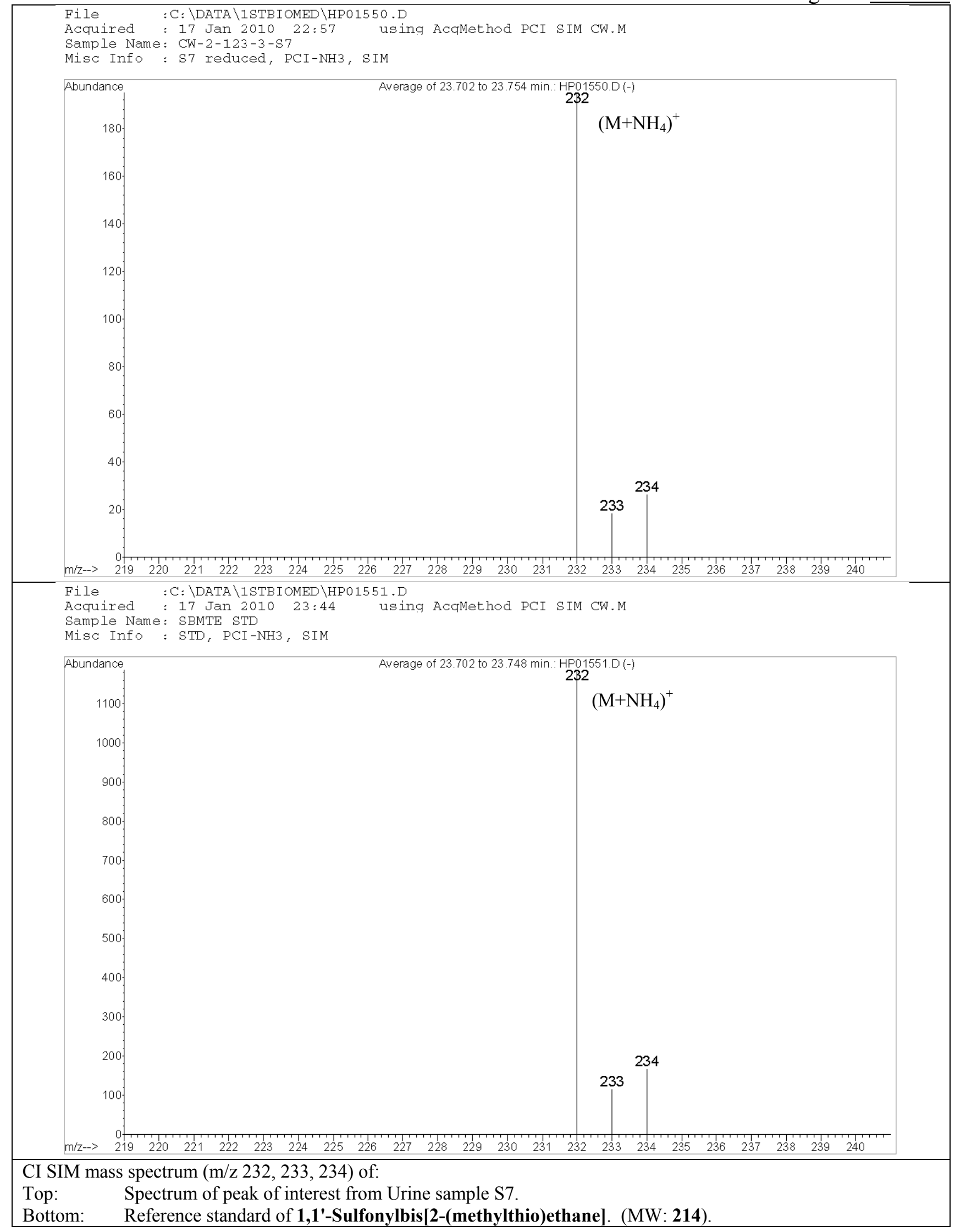


OPCW

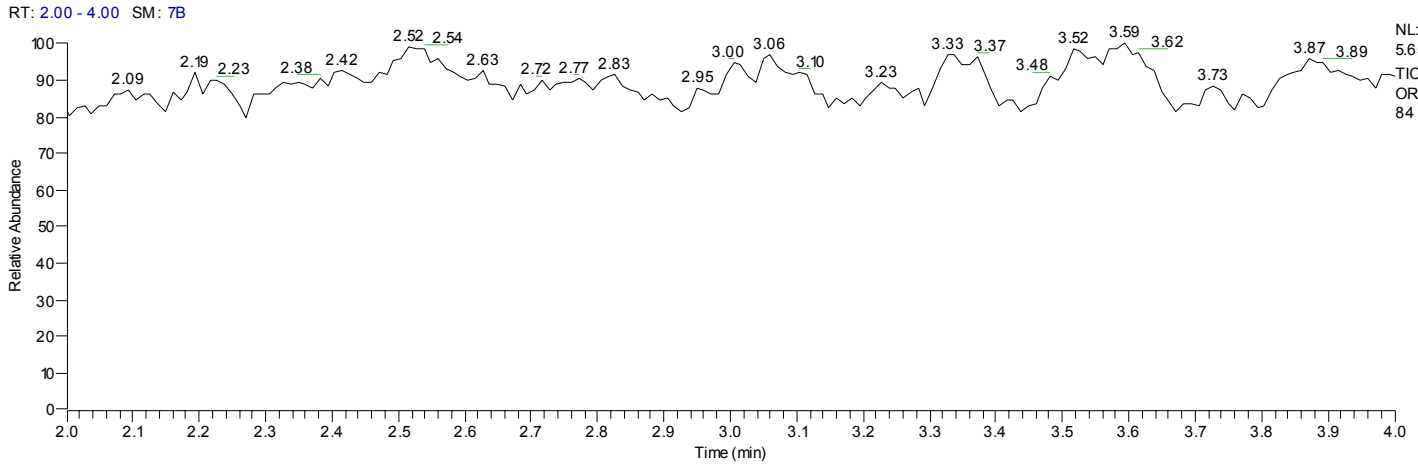

C:IXcaliburldatalORBI000885

1/17/2010 8:22:03 PM

CW-2-124-4-S1

Pos, MSMS, Sun column, 200uL/min

RT: $2.00-4.00$ SM: $7 \mathrm{~B}$

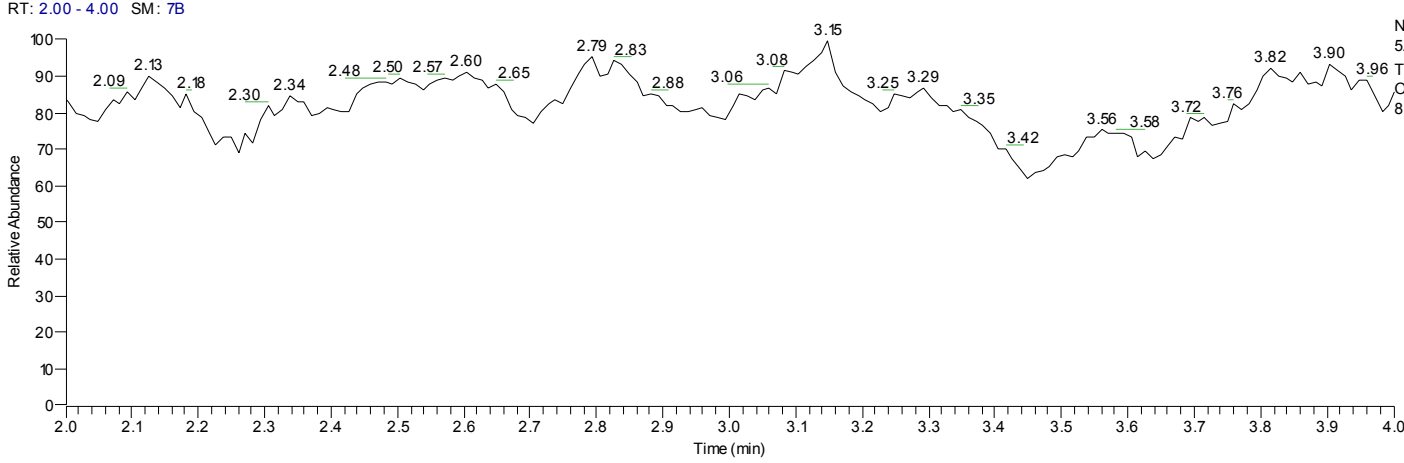

C:IXcaliburldatalORBI000886

1/17/2010 8:53:01 PM CW-2-123-6-S7

Pos, MSMS, Sun column, 200uL/min

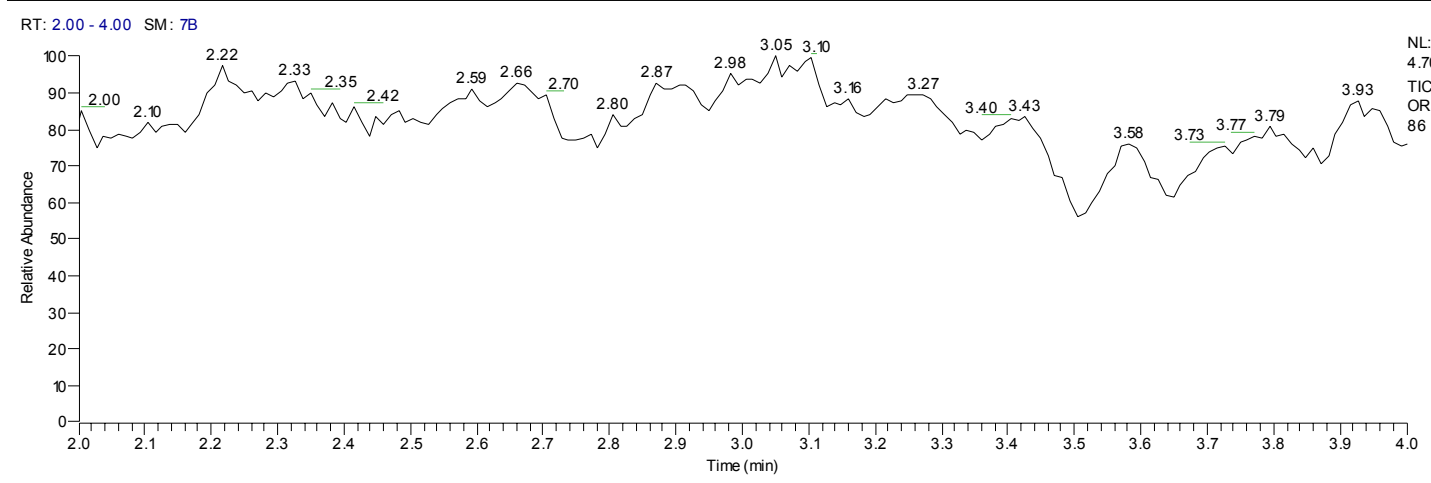

C:IXcaliburldatalORBI000887

1/17/2010 9:24:00 PM CW-2-114-3-STD (SBMSE)

Pos, MSMS, Sun column, 200uL/min

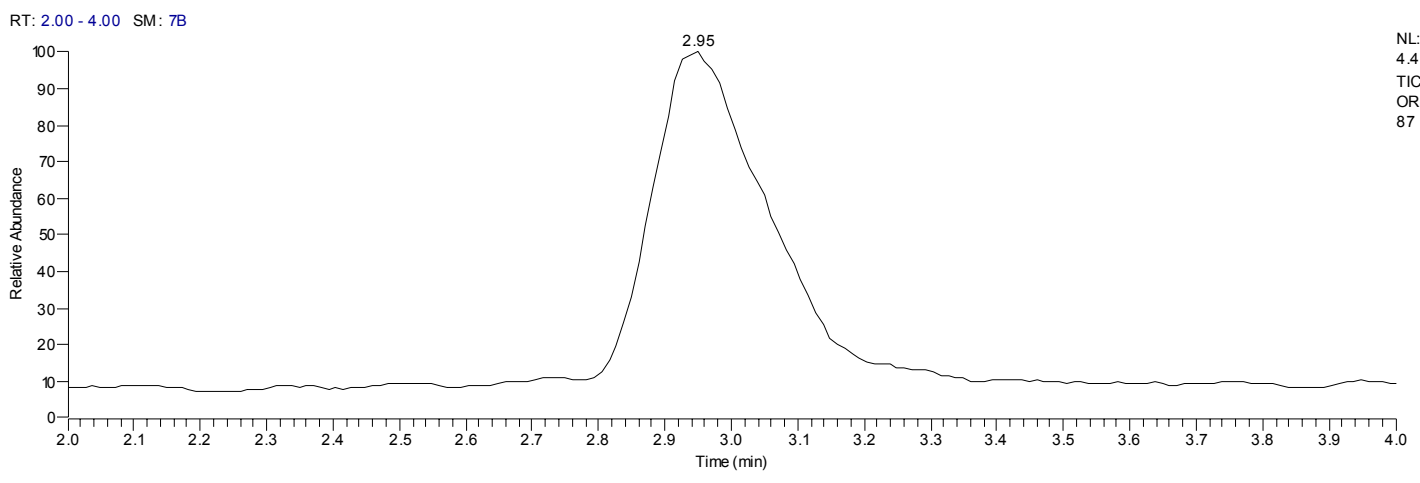

LC/MS/MS chromatograms. Top: water blank. Next: Urine blank S1. Third: Urine sample S7. Bottom: reference standard of 1,1'-Sulfonylbis[2-(methylsulfinyl)ethane]. 


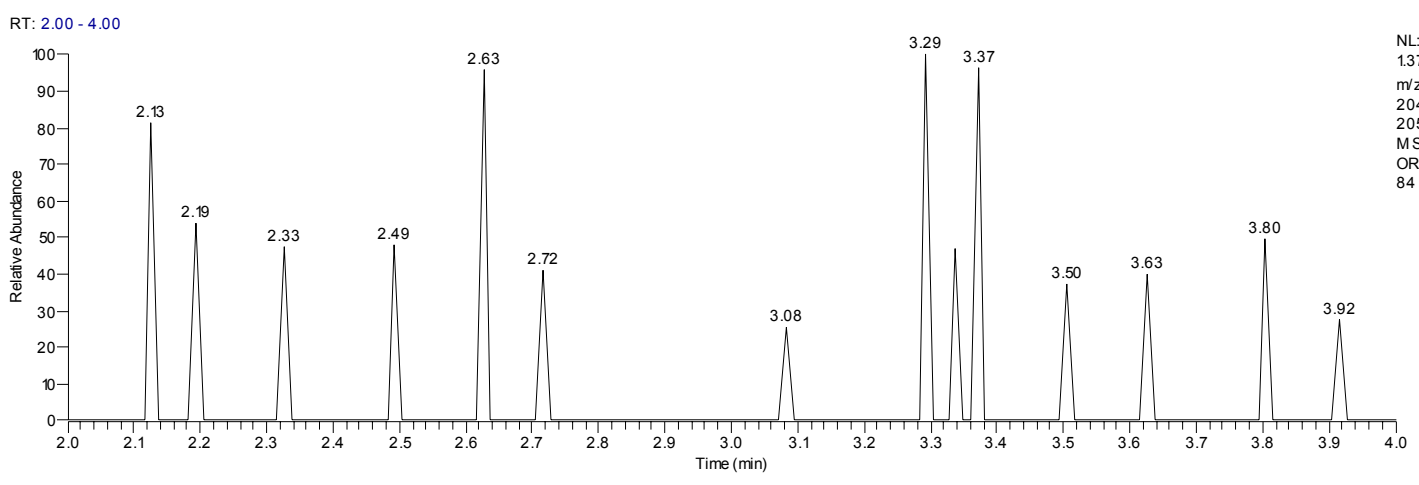

C:IXcaliburldatalORBI000885

1/17/2010 8:22:03 PM

CW-2-124-4-S1

Pos, MSMS, Sun column, 200uL/min

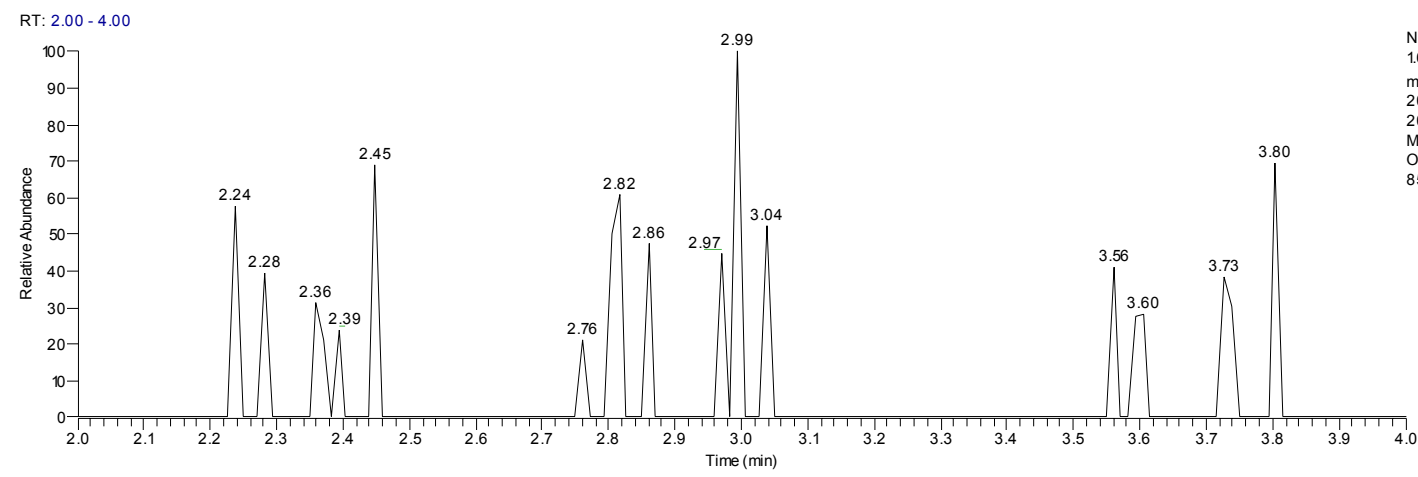

C:IXcaliburldatalORBI000886

1/17/2010 8:53:01 PM CW-2-123-6-S7

Pos, MSMS, Sun column, 200uL/min

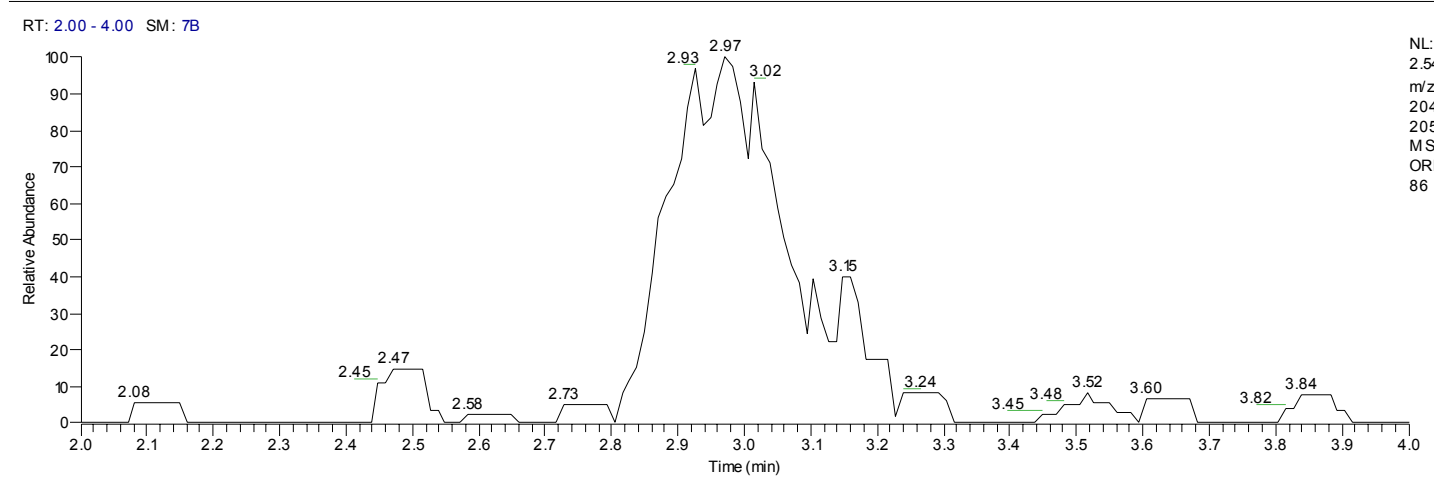

C:IXcaliburldatalORBI000887

1/17/2010 9:24:00 PM

CW-2-114-3-STD (SBMSE)

Pos, MSMS, Sun column, 200uL/min

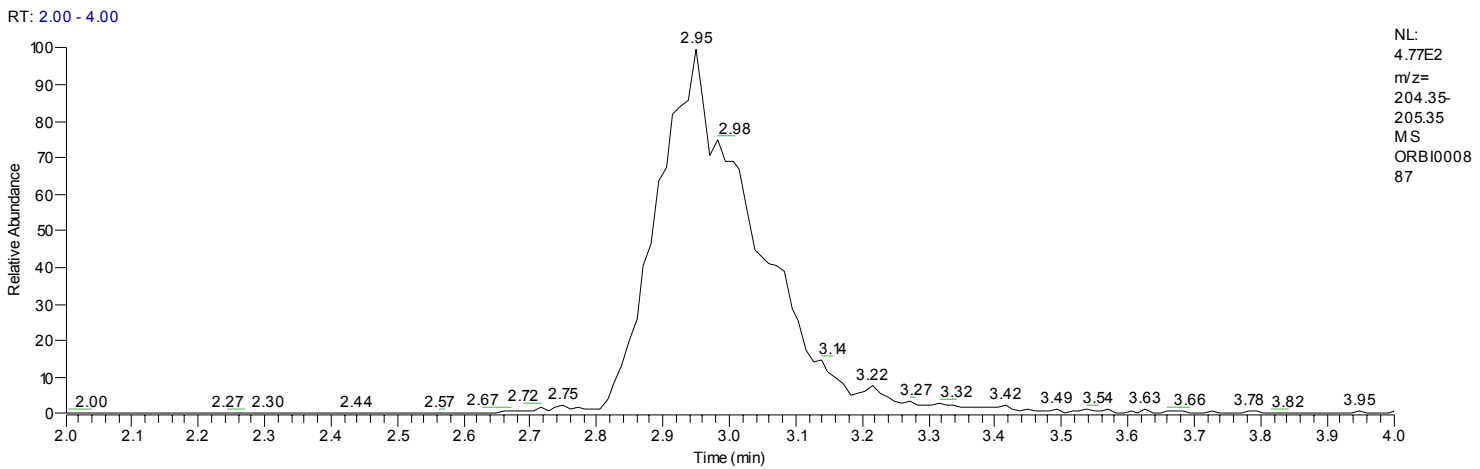

LC/MS/MS EIC (m/z 205) chromatograms. Top: water blank. Next: Urine blank S1. Third: Urine sample S5. Bottom: reference standard of 1,1'-Sulfonylbis[2-(methylsulfinyl)ethane]. 
ORBI000886 \#259-275 RT: 2.87-3.05 AV: 17 NL: $1.59 E 1$

T: ITMS + c ESI Full ms2 269.00@cid17.00 [70.00-280.00]

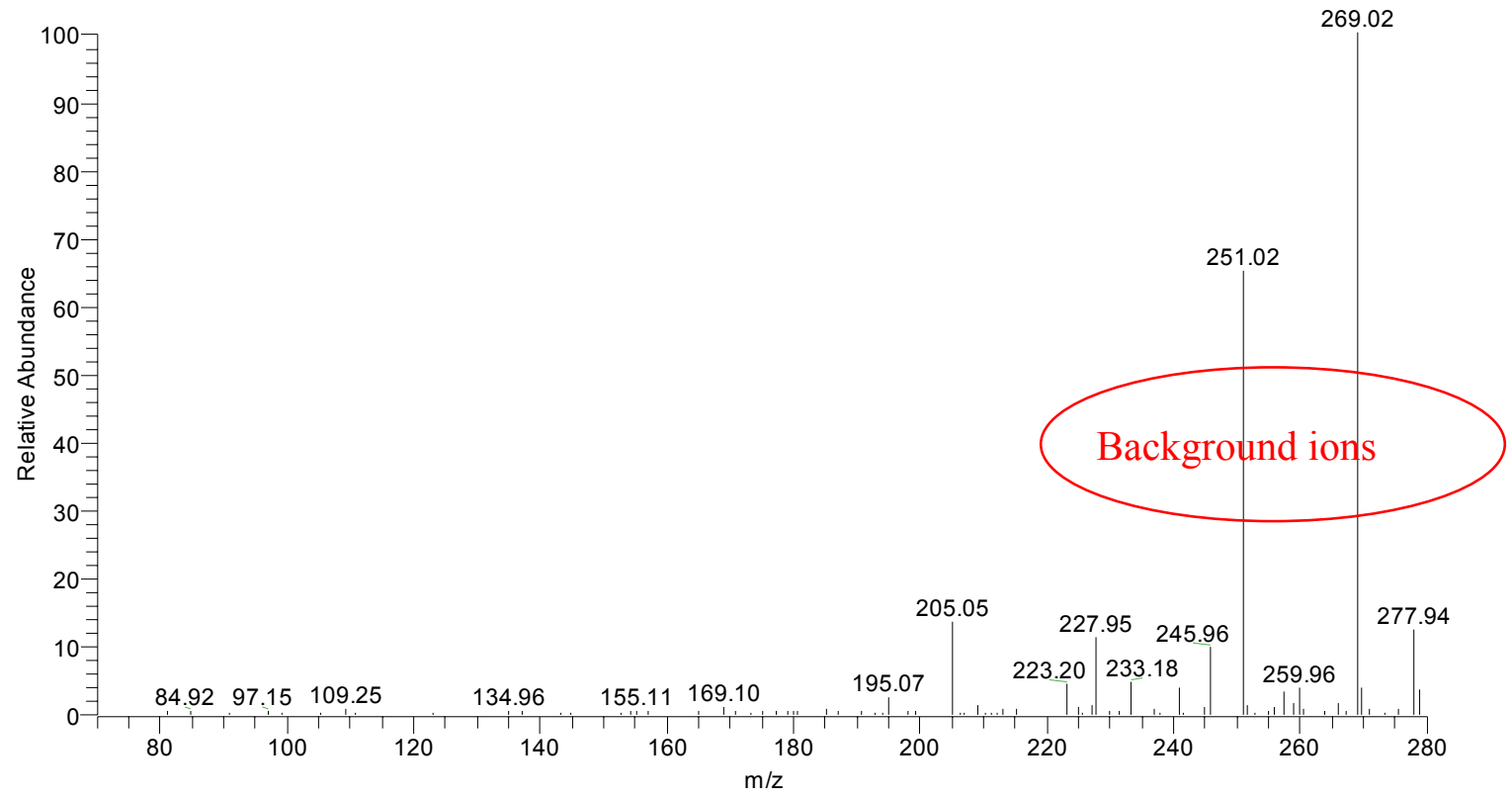

C:IXcaliburldatalORBI000887

1/17/2010 9:24:00 PM CW-2-114-3-STD (SBMSE)

Pos, MSMS, Sun column, 200uL/min

ORBI000887 \#259-276 RT: 2.87-3.06 AV: 18 NL: 3.16E2

T: ITMS + c ESI Full ms2 269.00@cid17.00 [70.00-280.00]

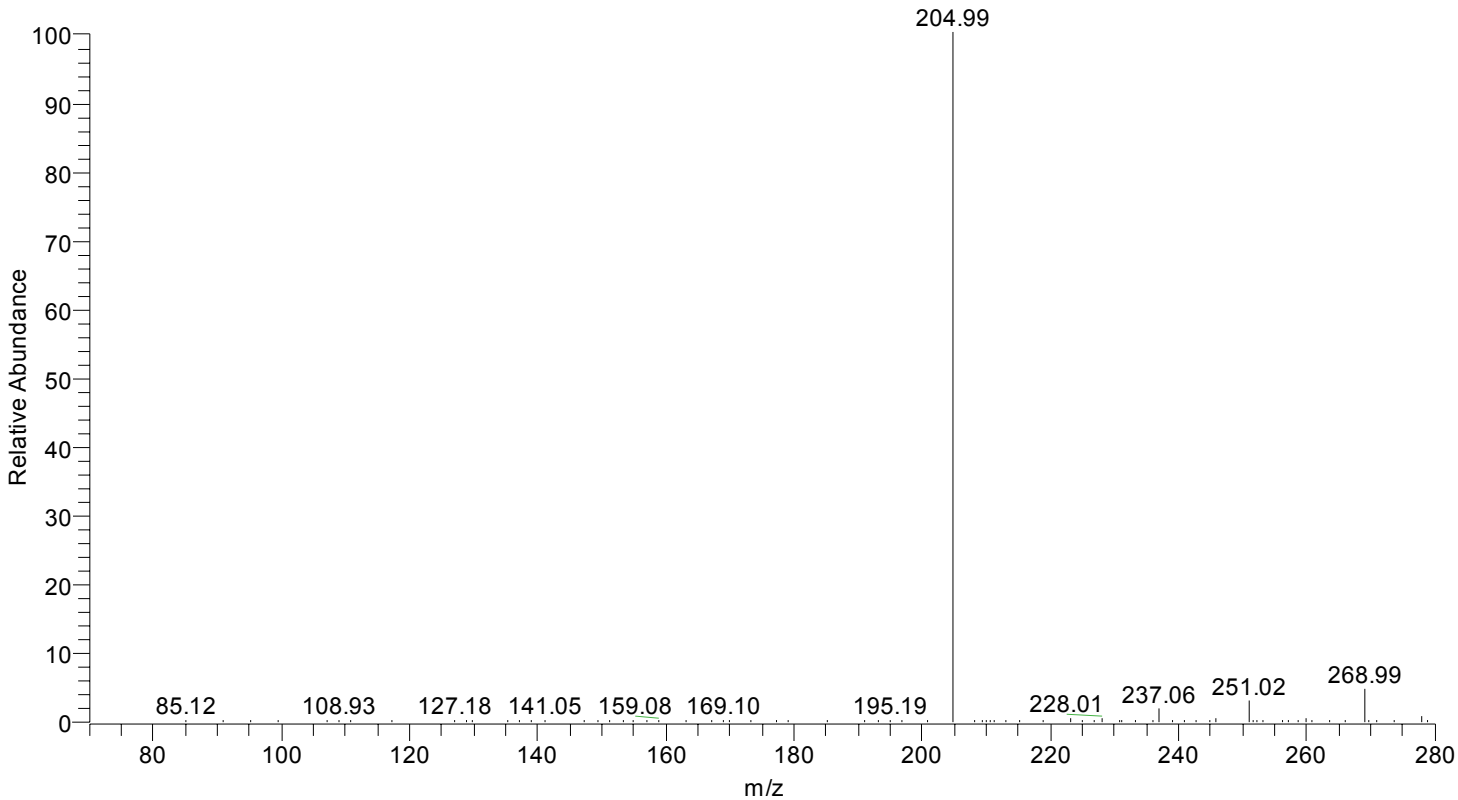

LC/MS/MS spectra - product spectra of parent $[\mathrm{M}+\mathrm{Na}]^{+} \mathrm{m} / \mathrm{z}$ 269. Top: Urine sample S5. Bottom: reference standard of 1,1'-Sulfonylbis[2-(methylsulfinyl)ethane]. 


\section{COMMENTS}

\section{General}

Samples were screened with GC/MS techniques, then confirmed with LC/MS.

2. Sample preparation

3. Analysis

No chemicals relevant to this scenario were present in sample 4.

4. Statement on the absence of derivatized chemicals(s) in original sample(s) and/or ambiguity of Lewisite(s) identification 